\title{
Hecke Algebras at Roots of Unity and Crystal Bases of Quantum Affine Algebras $\star$
}

\author{
Alain Lascoux ${ }^{1}$, Bernard Leclerc ${ }^{2}$, Jean-Yves Thibon ${ }^{3}$ \\ ${ }^{1}$ Institut Blaise Pascal, L I.T P, Université Paris 7, 2 place Jussieu, 75251 Paris Cedex 05, France. \\ ${ }^{2}$ Université de Caen, Département de Mathématiques, Esplanade de la Paix, BP 5186, 14032 Caen \\ Cedex, France \\ ${ }^{3}$ Institut Gaspard Monge, Université de Marne-la-Vallée, 2 rue de la Butte-Verte, 93166 Noisy-le- \\ Grand Cedex, France
}

Received: 10 November 1995/Accepted: 21 February 1996

\begin{abstract}
We present a fast algorithm for computing the global crystal basis of the basic $U_{q}\left(\widehat{\mathfrak{s l}}_{n}\right)$-module. This algorithm is based on combinatorial techniques which have been developed for dealing with modular representations of symmetric groups, and more generally with representations of Hecke algebras of type $A$ at roots of unity. We conjecture that, upon specialization $q \rightarrow 1$, our algorithm computes the decomposition matrices of all Hecke algebras at a $n^{\text {th }}$ root of 1 .
\end{abstract}

\section{Introduction}

This article arose as an attempt to understand the extensive numerical tables published by James in the appendix of [14]. These tables contain some decomposition matrices of $q$-Schur algebras at roots of unity, which contain as submatrices the decomposition matrices of Hecke algebras.

A major problem in the $p$-modular representation theory of the symmetric group $\Xi_{m}$ is to find an algorithm to compute its decomposition matrix. As a matter of fact, this matrix is naturally indexed by the same labels as the decomposition matrix of the Hecke algebra $H_{m}(\zeta)$, where $\zeta$ is a complex $p^{\text {th }}$ root of unity. As observed by James, these two matrices are very close to each other, although not equal in general, and apart from its independent interest, the computation of the decomposition matrices of Hecke algebras at roots of unity should be considered as an important step towards the modular case.

The decomposition matrix of $H_{m}(\zeta)$ is in principle expressible in terms of specializations at 1 of certain (inverse) affine Kazhdan-Lusztig polynomials. Unfortunately, these polynomials do not seem to be easily computable. The aim of this paper is to present a fast conjectural algorithm for calculating the decomposition matrices of Hecke algebras at roots of unity.

This algorithm was actually designed for solving a quite different problem, namely, to compute in an efficient way Kashiwara's global crystal basis of the

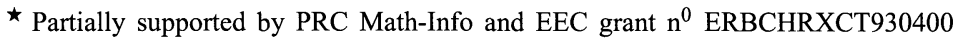


basic representation of the quantum affine algebra $U_{q}\left(\widehat{\mathfrak{s l}}_{n}\right)$. Determining the global basis is an important question in statistical mechanics, related to the diagonalization of the Hamiltonian of the XXZ model [6]. It turns out that our algorithm is directly inspired by the techniques described in [15] for computing the decomposition matrices of symmetric groups.

There are deep connections, essentially provided by Kazhdan-Lusztig theory, between modular representations, quantum groups and affine Lie algebras [31, 22]. However, in this case, we were rather guided by elementary combinatorial considerations.

The first hint that the Fock representation of $\widehat{\mathfrak{s l}}_{n}$ should have something to do with modular representations is the combinatorial description of this representation given in [5] in terms of Young diagrams. Indeed, one can observe that the raising and lowering operators $e_{r}, f_{r}$ are, from a combinatorial point of view, identical to Robinson's $r$-restricting and $r$-inducing operators [39].

A second evidence comes from comparison of the recent results of Kleshchev $[23,24,25]$ in the modular representation theory with those of Misra and Miwa [36] on the quantum group side. Indeed, Kleshchev has discovered a $p$-modular generalization of Young's branching rule for the irreducible representations of symmetric groups which is formulated in terms of a certain graph labelled by partitions. It turns out that this graph is exactly the crystal graph of the Fock representation of $U_{q}\left(\widehat{\mathfrak{s l}}_{p}\right)$ described by Misra and Miwa in [36].

This suggests that the crystal basis of the basic representation should play a rôle in the modular representation theory, and indeed, there is a strong experimental evidence, also supported by theoretical results, that the matrices expressing the global lower crystal basis of the basic representation of $U_{q}\left(\widehat{\mathfrak{s l}}_{n}\right)$ on the natural basis of the Fock space, are $q$-analogues of the decomposition matrices of the Hecke algebras at a primitive $n^{\text {th }}$ root of unity.

The conjecture can be extended to the case of the $q$-Schur algebra, by considering the whole Fock space as an irreducible $U_{q}\left(\widehat{\mathfrak{g l}}_{n}\right)$-module and introducing an appropriate canonical basis. As the description of this basis involves the introduction of new combinatorial ideas related to our previous work on ribbon tableaux [27], we will not touch this question here (see [30]).

A reasonable way of proving the conjecture would perhaps be to give an expression of the canonical basis in terms of affine Kazhdan-Lusztig polynomials, similar to the one given by $\mathrm{Du}[9,10]$ for $U_{q}\left(\mathfrak{s l}_{n}\right)$. In this case, our algorithm would probably provide a fast method for computing a subfamily of these polynomials.

This article is structured as follows. Sections 2 and 3 recall the necessary background on modular representations of symmetric groups and Hecke algebras at roots of unity. Section 4 describes the Fock representation of $U_{q}\left(\widehat{\mathfrak{s l}}_{n}\right)$, with conventions differing slightly from the usual ones, in order to be compatible with those of the modular representation theory. Section 5 describes, following [36], the crystal graph of the Fock representation, and gives some applications to the combinatorics of $n$ cores and $n$-quotients. Section 6 is devoted to the global lower crystal basis, and presents a fast algorithm to compute it. Some properties of the transition matrices are established, and the main conjecture is stated. Other properties of these matrices, also supporting the conjecture, are given in Sect. 7, where the MullineuxKleshchev involution is studied from the point of view of crystal bases. Section 8 is 
devoted to a reformulation of the main conjecture in terms of the upper crystal basis. Section 9 discusses, following an idea of Rouquier, a possible interpretation of our $q$-decomposition numbers in terms of the Jantzen filtration of a Specht module. Finally, as this paper could not have been written without the tables of James, we also conclude it by an appendix of tables giving the global crystal basis, the Gram matrices of the Shapovalov form (conjectured to provide $q$-analogues of the Cartan invariants of the Hecke algebras), and some examples of crystal graphs.

The main results of this paper have been announced in [28].

Note After the submission of this paper we have been informed by I Grojnowski and by S. Ariki that they could prove Conjecture 6.9 (ii). Recently, we have received a preprint of Ariki [2] in which a proof of a generalized version of Conjecture 6.9 (ii) involving cyclotomic Hecke algebras is presented. Grojnowski can deduce 6.9 (ii) from the results announced in [Gr].

To the best of our knowledge Conjecture 69 (i) and the interpretation of $q$-decomposition numbers proposed in Sect. 9 are still open.

\section{Modular Representations of Symmetric Groups}

We shall review some combinatorial aspects of the representation theory of symmetric groups over $\mathbb{F}_{p}=\mathbb{Z} / p \mathbb{Z}$. Our basic reference is [15].

2.1. The irreducible representations. To obtain representations of $\mathfrak{\Xi}_{m}$ over $\mathbb{F}_{p}$ one can start from the $p(m)$ ordinary irreducible representations $S(\lambda)$ written in Specht's form. (Here and in what follows, $p(m)$ denotes the number of partitions $\lambda$ of the integer $m$ ). The matrix coefficients in this form are integers and can be reduced modulo $p$. One gets in this way $p(m)$ representations $\overline{S(\lambda)}$ over $\mathbb{F}_{p}$. These are no longer irreducible in general.

Definition 2.1. A partition $\mu=\left(m^{\alpha_{m}}(m-1)^{\alpha_{m-1}} \cdots 2^{\alpha_{2}} 1^{\alpha_{1}}\right)$ is said to be p-regular if all its multiplicities $\alpha_{i}$ are $<p$. Otherwise it is called p-singular.

Theorem 2.2 (James). (i) Let $\mu$ be a p-regular partition of $m$. Then $\overline{S(\mu)}$ contains a unique maximal submodule $\operatorname{rad} \overline{S(\mu)}$. The simple module $\overline{S(\mu)} / \operatorname{rad} \overline{S(\mu)}$ is denoted by $D(\mu)$.

(ii) As $\mu$ varies over p-regular partitions of $m, D(\mu)$ varies over a complete set of inequivalent irreducible representations of $\mathfrak{S}_{m}$ over $\mathbb{F}_{p}$.

Thus the irreducible $p$-modular representations of $\Xi_{m}$ are labelled by $p$-regular partitions.

2.2. Branching rules. In characteristic 0, Young's branching rule tells us that

$$
S(\lambda) \downarrow \mathfrak{\Xi}_{\mathbb{S}_{m-1}}^{\mathfrak{S}_{m}} \cong \bigoplus_{v} S(v)
$$

where the sum is over all predecessors $v$ of $\lambda$ in the Young lattice. The corresponding problem in characteristic $p$ is to describe $D(\mu) \underset{\mathbb{S}_{m-1}}{\mathbb{S}_{m}}$ for a $p$-regular partition $\mu$. This is difficult because in general $D(\mu) \underset{\mathfrak{S}_{m-1}}{\mathbb{S}_{m}}$ is not semisimple, and its composition multiplicities are $>1$. 
Recently, Kleshchev [23, 24] solved the following simpler problem: to describe the socle (i.e. the sum of all simple submodules) of $D(\mu) \downarrow \downarrow_{\mathbb{S}_{m-1}}^{\mathfrak{S}_{m}}$.

Theorem 2.3 (Kleshchev). Let $\mu$ be a p-regular partition of $m$. Then

$$
\operatorname{Socle}\left(D(\mu) \downarrow \mathfrak{S}_{m-1}^{\mathfrak{S}_{m}}\right) \cong \bigoplus_{v} D(v)
$$

where the sum is over all predecessors $v$ of $\mu$ in Kleshchev's p-good lattice (to be described below). In particular the sum is multiplicity-free and its description is combinatorial.

Let us explain the construction of Kleshchev's $p$-good lattice. This is an infinite graph whose vertices are the $p$-regular partitions and whose edges correspond to the removal of a good node. To define what a good node is, we need some terminology.

Let $\lambda$ be a partition represented by its Young diagram. Let $N$ be the node (or box) at the intersection of row number $i$ and column number $j$. The content of $N$ is $c=j-i$, and its $p$-residue is $r=c \bmod p . N$ is called a $r$-node. For example, the nodes of $\lambda=\left(\begin{array}{ll}32 & 1^{2}\end{array}\right)$ have the following 3 -residues

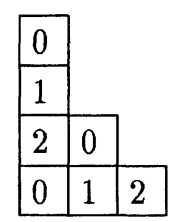

A removable node is a node of the boundary of $\lambda$ which can be removed, leaving a predecessor $v$ of $\lambda$ in Young's lattice. An indent node is a concave corner on the rim of $\lambda$ where a node can be added, giving a child of $\lambda$ in Young's lattice. For instance, $\lambda=\left(321^{2}\right)$ has three removable nodes (two have residue 0 , one has residue 2 ), and four indent nodes (one has residue 0 , one has residue 1 , and two have residue 2 ).

Fix a residue $r \in\{0,1, \ldots, p-1\}$ and consider the sequence of removable and indent $r$-nodes obtained by reading the boundary of $\lambda$ from left to right. Thus, for $\lambda=\left(\begin{array}{lll}3 & 2 & 1^{2}\end{array}\right)$ and $p=3$ one has:

- residue $r=0: R R I$

- residue $r=1: I$

- residue $r=2: I I R$

Given such a sequence of letters $R, I$, one can perform the following algorithm:

- read the sequence from left to right and put between brackets all pairs of consecutive letters $R I$,

- repeat the procedure ignoring the letters between brackets, until no pattern $R I$ is left.

The first $R$ outside the brackets (if any) then indicates the good node of $\lambda$ of residue $r$ (if any).

For example, this algorithm applied to the sequence 
goes through the following steps:

$$
\begin{gathered}
I R R(R I)(R I)(R I) I I I(R I) R R \\
I R(R(R I)(R I)(R I) I) I I(R I) R R \\
I(R(R(R I)(R I)(R I) I) I) I(R I) R R
\end{gathered}
$$

and the penultimate $R$ corresponds to a good node.

With this rule, we see that $\lambda=\left(321^{2}\right)$ has one good 2-node, no good 1-node, and one good 0 -node (the leftmost removable 0 -node).

Definition 2.4. Let $p$ be a prime number. The Kleshchev p-good lattice is the infinite graph whose vertices are the p-regular partitions $\mu$ and whose arrows are given by

$$
v \stackrel{i}{\longrightarrow} \mu \Longleftrightarrow v \text { is obtained from } \mu \text { by removing a good } i \text {-node . }
$$

Example 2.5. The Kleshchev 2-good lattice up to partitions of 5:

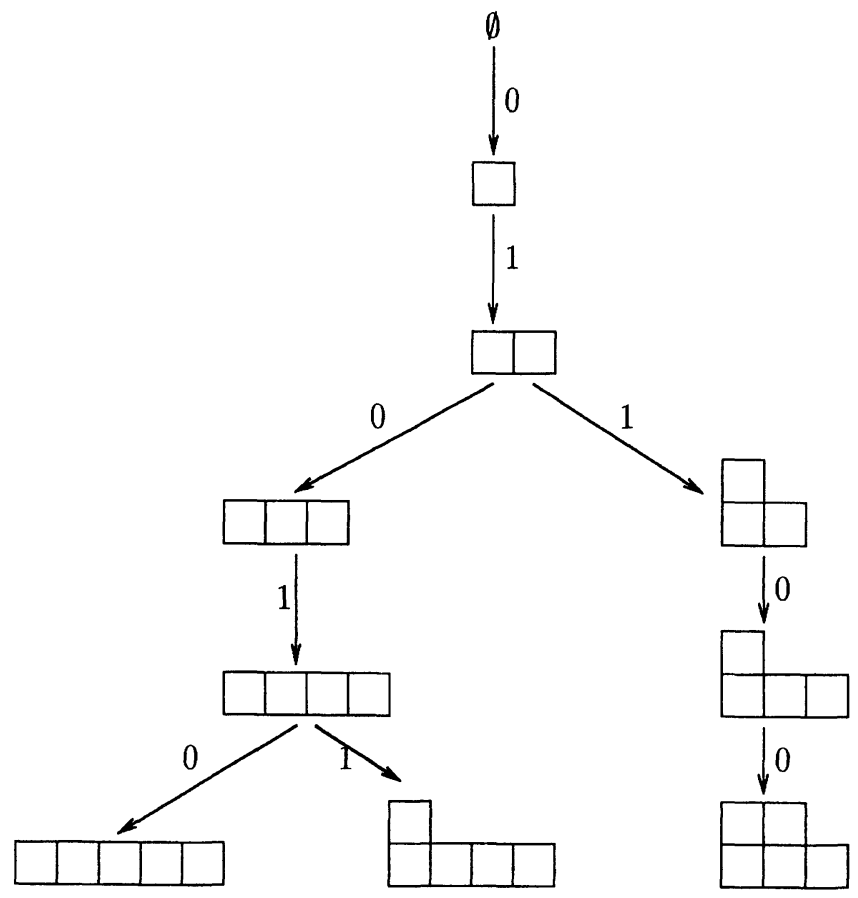

We should note that we have slightly reformulated Kleshchev's definition of a good node. It seems to us simpler this way. As a matter of fact, this bracketing procedure is a common operation on two-letter alphabets, and an essential ingredient in the definition of a symmetric group action on the set of Young tableaux (see [29]). Also it makes it obvious that Kleshchev's $p$-good lattice coincides with the crystal graph of the basic representation of the affine Lie algebra $\widehat{\mathfrak{s l}}_{p}$ described by Misra and Miwa [36] (see Sect. 5). 
It follows from the definition that each partition $\lambda$ has at most one good node of a given residue. On the other hand it is not difficult to see that $\lambda=$ $\left(m^{\alpha_{m}}(m-1)^{\alpha_{m-1}} \cdots 2^{\alpha_{2}} 1^{\alpha_{1}}\right)$ has no good node if and only if all multiplicities $\alpha_{i}$ are divisible by $p$. In particular, a nonempty $p$-regular partition $\mu$ has at least one good node. Moreover, if $v$ is $p$-regular and $\mu$ is obtained by removing a good node from $v$, then $v$ is also $p$-regular. Thus Kleshchev's lattice is the connected component of the empty partition in the graph $\Gamma_{p}$ whose set of vertices is the set of all partitions $\lambda$ and whose edges correspond to the removal of a good node. We shall see that $\Gamma_{p}$ is also the crystal graph of the Fock space of $\widehat{\mathfrak{s l}}_{p}$.

2.3. Decomposition matrices. Another basic problem in the $p$-modular representation theory of $\mathfrak{S}_{m}$ is to compute the composition multiplicities $d_{\lambda \mu}$ of the simple modules $D(\mu)$ in the $p$-reduced Specht modules $\overline{S(\lambda)}$. The nonnegative integers $d_{\lambda \mu}$ are called the decomposition numbers and the matrix $\mathbf{D}\left(\mathbb{F}_{p} \widetilde{S}_{m}\right)=\left[d_{\lambda_{\mu}}\right]$ is the decomposition matrix. For example for $p=2$ and $m=5$, this matrix is

$\begin{array}{llll}(5) & 1 & . & . \\ (41) & . & 1 & . \\ (32) & 1 & . & 1 \\ \left(31^{2}\right) & 2 & . & 1 \\ \left(2^{2} 1\right) & 1 & . & 1 \\ \left(21^{3}\right) & . & 1 & . \\ \left(1^{5}\right) & 1 & . & .\end{array}$

where - means 0 .

Although many properties of these matrices are known (see [15]) we still lack a general algorithm for computing them for all $m$ and $p$. The main properties are

Theorem 2.6. (i) $d_{\lambda \mu}=0$ unless $\lambda$ and $\mu$ have the same $p$-core,

(ii) $d_{\lambda_{\mu}}=0$ unless $\lambda \unlhd \mu$. Moreover, $d_{\mu \mu}=1$.

We recall that the $p$-core of $\lambda$ is the partition obtained by removing all possible $p$-ribbons (or $p$-rim-hooks) from the boundary of $\lambda$ (see [15]). Property (i) is known as Nakayama's conjecture, and was first proved by Brauer and Robinson (1947).

In (ii) $\unlhd$ denotes the usual dominance order on partitions, defined by

$$
\lambda \unlhd \mu \Longleftrightarrow \sum_{i=1}^{i=k} \lambda_{i} \leqq \sum_{i=1}^{i=k} \mu_{i} \text { for all } k \text {. }
$$

Denoting by $\leqq$ the lexicographic order, we see that $\lambda \unlhd \mu \Longrightarrow \lambda \leqq \mu$. Thus, if the rows and columns of $\mathbf{D}\left(\mathbb{F}_{p} \mathbb{\Xi}_{m}\right)$ are arranged in lexicographic order, (ii) means that $\mathbf{D}\left(\mathbb{F}_{p} \Im_{m}\right)$ has a "wedge shape," a fact first proved by Farahat, Peel, Müller (1976).

2.4. Grothendieck groups. It is useful to reformulate the definition of the decomposition matrices in the language of Grothendieck groups (see [4], chapter 2).

Let $F$ be a field. We let $G_{0}\left(F \Im_{m}\right)$ denote the Grothendieck group of the category of finitely generated $F \Im_{m}$-modules. It is a free abelian group with basis the set $\left\{\left[D_{i}\right]\right\}$ of classes of simple $F \mathfrak{\Im}_{m}$-modules $D_{i}$. Let also $K_{0}\left(F \Im_{m}\right)$ denote the Grothendieck group of the category of finitely generated projective $F \mathfrak{\Xi}_{m}$-modules. 
It is a free abelian group with basis the set $\left\{\left[Y_{i}\right]\right\}$ of classes of indecomposable summands $Y_{i}$ of the regular representation $F \Im_{m}$. The indexation of these bases is such that $D_{i} \cong Y_{i} / \operatorname{rad} Y_{i}$, where $\operatorname{rad} Y_{i}$ is the unique maximal submodule of $Y_{i}$. There is a natural pairing between $K_{0}\left(F \Im_{m}\right)$ and $G_{0}\left(F \Im_{m}\right)$ given by

$$
\left(\left[Y_{i}\right],\left[D_{j}\right]\right)=\delta_{i j} .
$$

It is customary to consider at the same time the Grothendieck groups associated with all symmetric groups (see [40]) and to set

$$
\mathcal{G}(F)=\bigoplus_{m} G_{0}\left(F \Im_{m}\right), \quad \mathcal{K}(F)=\bigoplus_{m} K_{0}\left(F \Im_{m}\right)
$$

The pairing above is extended to $\mathcal{K}(F) \times \mathcal{G}(F)$ by requiring that

$$
\left(K_{0}\left(F \mathfrak{\Im}_{m}\right), G_{0}\left(F \mathfrak{\Im}_{l}\right)\right)=0 \text { if } m \neq l \text {. }
$$

Also, $\mathcal{G}(F)$ and $\mathcal{K}(F)$ are made into rings by introducing an additional multiplication corresponding to the induction from $\mathfrak{\Xi}_{m} \times \mathfrak{\Xi}_{l}$ to $\mathfrak{\Xi}_{m+l}$, that is, for a $F \mathfrak{\Xi}_{m}$-module $M$ and a $F \mathfrak{\Xi}_{l}$-module $N$, one sets

$$
[M][N]=\left[M \otimes N \uparrow \Im_{m+l}^{\Xi_{m} \times \Im_{l}}\right] .
$$

If $F=\mathbb{Q}$ the field of rational numbers, it is well-known that

$$
\mathcal{G}(F) \cong \mathcal{K}(F) \cong \operatorname{Sym},
$$

the ring of integral linear combinations of Schur symmetric functions $s_{\lambda}$ in an infinite number of variables (the reader is referred to [32] for definitions relative to symmetric functions). The isomorphism is the $\mathbb{Z}$-linear map defined by

$$
[S(\lambda)] \longrightarrow s_{\lambda}
$$

The above pairing coincides with the usual scalar product on Sym:

$$
\left(s_{\lambda}, s_{\mu}\right)=\delta_{\lambda \mu}
$$

In the case $F=\mathbb{F}_{p}$, the rings $\mathcal{G}(F)$ and $\mathcal{K}(F)$ can still be interpreted in terms of symmetric functions. Let $\mathcal{J}^{(p)}$ be the ideal of Sym generated by the power sums $p_{k}$ with $k$ divisible by $p$. Then the map

$$
[\overline{S(\lambda)}] \longrightarrow d\left(s_{\lambda}\right):=s_{\lambda} \bmod \mathcal{J}^{(p)}
$$

extends to a ring isomorphism from $\mathcal{G}\left(\mathbb{F}_{p}\right)$ to $S y m / \mathcal{J}^{(p)}$. On the other hand, $\mathcal{K}\left(\mathbb{F}_{p}\right)$ is isomorphic to $\mathcal{T}^{(p)}=\left(\mathcal{J}^{(p)}\right)^{\perp}$, the orthogonal complement of $\mathcal{J}^{(p)}$ in Sym with regard to $(\cdot, \cdot)$. The pairing $(\cdot, \cdot)_{p}$ between $\mathcal{K}\left(\mathbb{F}_{p}\right)$ and $\mathcal{G}\left(\mathbb{F}_{p}\right)$ is the one naturally induced by $(\cdot, \cdot)$, that is,

$$
(u, d(v))_{p}=(u, v), \quad u \in \mathcal{T}^{(p)}, v \in S y m,
$$

where $d(v):=v \bmod \mathcal{J}^{(p)}$. Let $\mu$ be a $p$-regular partition of $m$. Let $\phi_{\mu} \in S y m / \mathcal{J}^{(p)}$ and $\eta_{\mu} \in \mathcal{T}^{(p)}$ denote the images of the classes $[D(\mu)] \in G_{0}\left(\mathbb{F}_{p} \Im_{m}\right)$ and $[Y(\mu)] \in$ 
$K_{0}\left(\mathbb{F}_{p} \Im_{m}\right)$ under these isomorphisms. We have

$$
\begin{gathered}
d\left(s_{\lambda}\right)=\sum_{\mu} d_{\lambda \mu} \phi_{\mu}, \\
\left(\eta_{\mu}, \phi_{v}\right)_{p}=\delta_{\lambda \mu},
\end{gathered}
$$

and therefore $d_{\lambda \mu}=\left(\eta_{\mu}, d\left(s_{\lambda}\right)\right)_{p}=\left(\eta_{\mu}, s_{\lambda}\right)$. This yields

$$
\eta_{\mu}=\sum_{\lambda} d_{\lambda \mu} s_{\lambda}
$$

Hence the decomposition matrix $\mathbf{D}\left(\mathbb{F}_{p} \mathfrak{S}_{m}\right)$ may be regarded either as the matrix of the natural embedding of $K_{0}\left(\mathbb{F}_{p} \mathbb{S}_{m}\right)$ into $G_{0}\left(\mathbb{Q} \mathfrak{\Im}_{m}\right)$ in the bases $\left\{\eta_{\mu}\right\},\left\{s_{\lambda}\right\}$, or as the transpose of the reduction $\bmod p$ (or decomposition map) from $G_{0}\left(\mathbb{Q} \mathfrak{S}_{m}\right)$ to $G_{0}\left(\mathbb{F}_{p} \Xi_{m}\right)$ in the bases $\left\{s_{\lambda}\right\},\left\{\phi_{\mu}\right\}$. The composition of these two maps is known as the Cartan map. Its matrix $\mathbf{C}\left(\mathbb{F}_{p} \widetilde{\Xi}_{m}\right)$ in the bases $\left\{\eta_{\mu}\right\},\left\{\phi_{\mu}\right\}$ is given by

$$
\mathbf{C}\left(\mathbb{F}_{p} \Im_{m}\right)={ }^{\operatorname{tr}} \mathbf{D}\left(\mathbb{F}_{p} \Im_{m}\right) \mathbf{D}\left(\mathbb{F}_{p} \Im_{m}\right) \text {. }
$$

In particular it is a symmetric matrix. Its entries may be expressed as

$$
c_{\mu \nu}=\sum_{\lambda} d_{\lambda \mu} d_{\lambda v}=\left(\eta_{\mu}, \eta_{v}\right) .
$$

We can now state the problem of the computation of the decomposition numbers in the following way. The rings $\mathcal{T}^{(p)}$ and $S y m / \mathcal{J}^{(p)}$ are endowed with canonical $\mathbb{Z}$-bases $\left\{\eta_{\mu}\right\}$ and $\left\{\phi_{\mu}\right\}$ coming from their interpretation as Grothendieck groups in characteristic $p$. These bases are dual with respect to the natural scalar product of Sym. One would like to compute the transition matrices from these bases to the basis $\left\{s_{\lambda}\right\}$ of Schur functions.

2.5. $r$-inducing and r-restricting operators. We introduce now some linear operators on $S y m$ which are fundamental tools for computing the decomposition matrices $\mathbf{D}\left(\mathbb{F}_{p} \mathfrak{S}_{m}\right)$. They were first studied by Robinson ([39], Chap. 6).

For $r=0,1, \ldots, p-1$, define the linear operators $e_{r}$ by

$$
e_{r} s_{v}=\sum_{\lambda} s_{\lambda}
$$

where the sum is over all partitions $\lambda$ obtained by removing a $r$-node of $v$. Similarly, define $f_{r}$ by

$$
f_{r} s_{\lambda}=\sum_{v} s_{v}
$$

where the sum is over all partitions $v$ obtained by adding a $r$-node to $\lambda$. The operators $e_{r}$ and $f_{r}$ are called respectively the $r$-restricting and $r$-inducing operators.

Lemma 2.7. Let $\mathcal{S}^{(p)}$ denote the subring of Sym generated by the power sums $p_{k}$ for $k$ divisible by $p$.

(i) For $P \in \mathcal{S}^{(p)}, Q \in S y m$, one has

$$
e_{r}(P Q)=P e_{r}(Q), \quad f_{r}(P Q)=P f_{r}(Q) .
$$

(ii) For $P, Q \in$ Sym, one has

$$
\left(e_{r}(P), Q\right)=\left(P, f_{r}(Q)\right) .
$$


Proof. (i) It is enough to check the statement in the particular case $P=p_{k p}$ and $Q=s_{\lambda}$, and this is a consequence of the well-known combinatorial description of the Schur-expansion of $p_{k p} s_{\lambda}$ (see [32], I 3, Ex. 11). We omit the details.

(ii) Reduce to $P=s_{\lambda}$ and $Q=s_{v}$, in which case it results easily from the definitions of $(\cdot, \cdot), e_{r}$ and $f_{r}$.

It follows from (i) that the ideal $\mathcal{J}^{(p)}$ is stable under $e_{r}$ and $f_{r}$. Therefore $e_{r}$ and $f_{r}$ induce linear operators on $\mathcal{G}\left(\mathbb{F}_{p}\right) \cong S y m / \mathcal{J}^{(p)}$. Also, (ii) shows that $\mathcal{K}\left(\mathbb{F}_{p}\right) \cong \mathcal{T}^{(p)}$ is invariant under the action of $e_{r}$ and $f_{r}$. By abuse of notations, we shall still denote by $e_{r}$ and $f_{r}$ these endomorphisms of $\mathcal{G}\left(\mathbb{F}_{p}\right)$ and $\mathcal{K}\left(\mathbb{F}_{p}\right)$.

The origin of these operators comes from the description of the blocks of $\mathbb{F}_{p} \mathfrak{\Xi}_{m}$ in terms of $p$-cores given by the Nakayama conjecture. Indeed if $v, v^{\prime}$ are two partitions obtained from $\lambda$ by filling indent nodes $\gamma, \gamma^{\prime}$ (resp. by removing removable nodes $\gamma, \gamma^{\prime}$ ), then $v$ and $v^{\prime}$ have the same $p$-core if and only if $\gamma$ and $\gamma^{\prime}$ have the same residue $r$. Therefore, if $M$ is a $\mathbb{F}_{p} \Im_{m}$-module contained in a block, the $r$-inducing operators $f_{r}$ of $\mathcal{G}\left(\mathbb{F}_{p}\right)$ and $\mathcal{K}\left(\mathbb{F}_{p}\right)$ compute the classes $f_{r}[M]$ of the projections of the induced module $M \uparrow \mathfrak{S}_{m+1}^{\mathfrak{S}_{m+1}}$ on the various blocks of $\mathbb{F}_{p} \mathfrak{S}_{m+1}$. The interpretation of the $r$-restricting operators $e_{r}$ is similar.

\section{Representations of the Hecke Algebras of Type $A$ at Roots of Unity}

Let $H_{m}(v)$ denote the Hecke algebra of type $A$ over $\mathbb{C}(v)$. This is the associative algebra with 1 generated by the symbols $T_{i}, i=1, \ldots, m-1$ subject to the relations

$$
\begin{aligned}
T_{i} T_{j} & =T_{j} T_{i} \quad|i-j|>1, \\
T_{i} T_{j} T_{i} & =T_{j} T_{i} T_{j} \quad|i-j|=1, \\
T_{i}^{2} & =(v-1) T_{i}+v .
\end{aligned}
$$

Let $z \in \mathbb{C}$ and let $H_{m}(z)$ be the algebra obtained by specializing $v \rightarrow z$. For generic $z$ (i.e. $z \neq$ a root of unity or 0 ) it is known that $H_{m}(z) \cong \mathbb{C} \mathfrak{S}_{m}$. Throughout this paper we shall denote by $\zeta$ a primitive $n^{\text {th }}$ root of 1 . When $n=p$ is a prime, the representation theory of $H_{m}(\zeta)$ is in many respects similar to the $p$-modular representation theory of $\mathfrak{\Xi}_{m}$, as will be illustrated in this section. Here, our main references are $[7,8]$.

3.1. Irreducible modules. The natural $v$-analogues of the Specht modules for $H_{m}(v)$ have been described in [7] (see also [11] for a polynomial realization of these modules). We shall still denote them by $S(\lambda)$. The $S(\lambda), \lambda \vdash m$ form a complete set of nonequivalent simple $H_{m}(v)$-modules, and the matrix coefficients in these representations are Laurent polynomials in $v$. Hence it is possible to specialize $v$ to $\zeta$ and to get Specht modules $\overline{S(\lambda)}$ for $H_{m}(\zeta)$. These are in general no longer irreducible.

Theorem 3.1 (Dipper, James). (i) Let $\mu$ be a n-regular partition of $m$. Then $\overline{S(\mu)}$ contains a unique maximal submodule $\operatorname{rad} \overline{S(\mu)}$. The simple module $\overline{S(\mu)} / \operatorname{rad} \overline{S(\mu)}$ is denoted by $D(\mu)$.

(ii) As $\mu$ varies over $n$-regular partitions of $m, D(\mu)$ varies over a complete set of inequivalent irreducible representations of $H_{m}(\zeta)$. 
Thus the irreducible representations of $H_{m}(\zeta)$ are labelled by $n$-regular partitions.

3.2. Decomposition matrices. One defines as in the case of the symmetric group the decomposition matrix $\mathbf{D}\left(H_{m}(\zeta)\right)$ of composition multiplicities of the reduced Specht modules $\overline{S(\lambda)}$. For typographical convenience, we shall still denote its entries by $d_{\lambda \mu}$ but these should not be confused when $n=p$ is a prime with the decomposition numbers of the symmetric groups, with which they do not coincide in general. For example the matrix $\mathbf{D}\left(H_{5}(-1)\right)$ is

$\begin{array}{llll}(5) & 1 & . & . \\ (41) & \cdot & 1 & . \\ (32) & . & \cdot & 1 \\ \left(31^{2}\right) & 1 & \cdot & 1 \\ \left(2^{2} 1\right) & \cdot & . & 1 \\ \left(21^{3}\right) & . & 1 & . \\ \left(1^{5}\right) & 1 & \cdot & .\end{array}$

which is different from the matrix $\mathbf{D}\left(\mathbb{F}_{2} \mathfrak{S}_{5}\right)$ shown in the previous section. However there are some striking similarities. Thus,

Theorem 3.2 (Dipper, James). (i) $d_{\lambda \mu}=0$ unless $\lambda$ and $\mu$ have the same $n$-core,

(ii) $d_{\lambda \mu}=0$ unless $\lambda \unlhd \mu$. Moreover, $d_{\mu \mu}=1$.

In [14], James has proposed to regard the matrix $\mathbf{D}\left(H_{m}(\zeta)\right)$ (where $n=p$ ) as a "first approximation" of $\mathbf{D}\left(\mathbb{F}_{p} \Im_{m}\right)$. Indeed, it is shown in [14] that

$$
\mathbf{D}\left(\mathbb{F}_{p} \Im_{m}\right)=\mathbf{D}\left(H_{m}(\zeta)\right) \cdot A,
$$

where $A=\left(a_{\mu \nu}\right)$ is a unitriangular adjustment matrix with nonnegative entries, most of the off-diagonal $a_{\mu \nu}$ being 0 . It is even conjectured in [14] that

$$
\mathbf{D}\left(\mathbb{F}_{p} \mathfrak{\Xi}_{m}\right)=\mathbf{D}\left(H_{m}(\zeta)\right) \text { if } m<p^{2} .
$$

3.3. Grothendieck groups, $r$-inducing and r-restricting operators. Let $H_{m}$ denote $H_{m}(v)$ or $H_{m}(z)$ for some $z \in \mathbb{C}$. We denote by $G_{0}\left(H_{m}\right)$ (resp. $K_{0}\left(H_{m}\right)$ ) the Grothendieck group of the category of finitely generated $H_{m}$-modules (resp. finitely generated projective $H_{m}$-modules). This is a free abelian group with basis the set $\left\{\left[D_{i}\right]\right\}$ of classes of simple $H_{m}$-modules $D_{i}$ (resp. the set $\left\{\left[Y_{i}\right]\right\}$ of classes of indecomposable summands $Y_{i}$ of $\left.H_{m}\right)$, and $D_{i} \cong Y_{i} / \operatorname{rad} Y_{i}$. There is a natural pairing between $K_{0}\left(H_{m}\right)$ and $G_{0}\left(H_{m}\right)$ given by

$$
\left(\left[Y_{i}\right],\left[D_{j}\right]\right)=\delta_{i j}
$$

As in the case of the symmetric group, we shall consider at the same time the Grothendieck groups associated with all Hecke algebras $H_{m}$ and set

$$
\mathcal{G}=\bigoplus_{m} G_{0}\left(H_{m}\right), \quad \mathcal{K}=\bigoplus_{m} K_{0}\left(H_{m}\right) .
$$

The pairing above is extended to $\mathcal{K} \times \mathcal{G}$ by requiring that

$$
\left(K_{0}\left(H_{m}\right), G_{0}\left(H_{l}\right)\right)=0 \text { if } m \neq l \text {. }
$$


Also, $\mathcal{G}$ and $\mathcal{K}$ are made into rings by introducing an additional multiplication corresponding to the induction from $H_{m} \otimes H_{l}$ to $H_{m+l}$. When $H_{m}=H_{m}(v)$, we have

$$
\mathcal{G}(v) \cong \mathcal{K}(v) \cong S y m,
$$

the isomorphism still being given by

$$
[S(\lambda)] \longrightarrow s_{\lambda} .
$$

In the case $H_{m}=H_{m}(\zeta)$, the map

$$
[\overline{S(\lambda)}] \longrightarrow d\left(s_{\lambda}\right):=s_{\lambda} \bmod \mathcal{J}^{(n)}
$$

extends to a ring isomorphism from $\mathcal{G}(\zeta)$ to $\operatorname{Sym} / \mathcal{J}^{(n)}$. On the other hand, $\mathcal{K}(\zeta)$ is isomorphic to $\mathcal{T}^{(n)}$. The pairing $(\cdot, \cdot)_{n}$ between $\mathcal{K}(\zeta)$ and $\mathcal{G}(\zeta)$ is the one naturally induced by $(\cdot, \cdot)$, that is,

$$
(u, d(v))_{n}=(u, v), \quad u \in \mathcal{T}^{(n)}, v \in S y m,
$$

where $d(v):=v \bmod \mathcal{J}^{(n)}$. Let $\mu$ be a $n$-regular partition of $m$. Let $\phi_{\mu} \in \operatorname{Sym} / \mathcal{J}^{(n)}$ and $\eta_{\mu} \in \mathcal{T}^{(n)}$ denote the images of the classes $[D(\mu)] \in G_{0}\left(H_{m}(\zeta)\right)$ and $[Y(\mu)] \in$ $K_{0}\left(H_{m}(\zeta)\right)$ under these isomorphisms. Then, $\left\{\phi_{\mu}\right\}$ and $\left\{\eta_{\mu}\right\}$ are linear bases of $\operatorname{Sym} / \mathcal{J}^{(n)}$ and $\mathcal{T}^{(n)}$ respectively, satisfying equations

$$
\begin{gathered}
d\left(s_{\lambda}\right)=\sum_{\mu} d_{\lambda \mu} \phi_{\mu}, \\
\left(\eta_{\mu}, \phi_{v}\right)_{n}=\delta_{\lambda \mu}, \\
\eta_{\mu}=\sum_{\lambda} d_{\lambda \mu} s_{\lambda} .
\end{gathered}
$$

The problem is to compute the transition matrices from these bases to the basis $\left\{s_{\lambda}\right\}$ of Schur functions. (Again, we have deliberately used for simplicity the same notations $\phi_{\mu}, \eta_{\mu}$ as in Sect. 2, but when $n=p$ they indicate in general different symmetric functions.)

Finally, the linear operators $e_{r}, f_{r}$ on Sym defined in 2 in connection with a prime $p$ still make sense with $p$ replaced by $n$. The results of Lemma 2.7 are still valid (with $p$ replaced by $n$ ), so that the operators $e_{r}, f_{r}$ induce endomorphisms of $\mathcal{G}(\zeta)$ and $\mathcal{K}(\zeta)$ still denoted by $e_{r}, f_{r}$. These are the r-restricting and $r$-inducing operators which can again be interpreted as the combination of restricting (or inducing) and projecting onto the different blocks.

3.4. Branching rules. The definition of Kleshchev's $p$-good lattice does not use the fact that $p$ is a prime. Hence it makes sense to consider the $n$-good lattice of $n$ regular partitions for arbitrary $n \geqq 2$. It may be conjectured that this graph describes the socles of the restricted modules

$$
D(\mu) \downarrow_{H_{m-1}(\zeta)}^{H_{m}(\zeta)} .
$$

In any case, we shall see in the next sections that this graph (for arbitrary $n$ ) does appear in the context of the representation theory of the quantized affine Lie algebra $U_{q}\left(\widehat{\mathfrak{s}}_{n}\right)$. 


\section{The Basic Representation of $U_{q}\left(\widehat{\mathfrak{s l}}_{n}\right)$}

In this section we describe, following [36], the Fock representation of the quantized affine Lie algebra $U_{q}\left(\widehat{\mathfrak{s l}}_{n}\right)$, and the basic representation $M\left(\Lambda_{0}\right)$ embedded in it. We note that $M\left(\Lambda_{0}\right)$ can be regarded as a $q$-analogue of the Grothendieck ring $\mathcal{K}(\zeta)$ of the previous section, with the raising and lowering operators of $U_{q}\left(\widehat{\mathfrak{s l}}_{n}\right)$ acting as $q$-analogues of Robinson's $r$-restricting and $r$-inducing operators.

4.1. Highest weight $U_{q}\left(\widehat{\mathfrak{s l}}_{n}\right)$-modules. We first recall the definition of $U_{q}\left(\widehat{\mathfrak{s l}}_{n}\right)$. Let $\mathfrak{h}$ be a $(n+1)$-dimensional vector space over $\mathbb{Q}$ with basis $\left\{h_{0}, h_{1}, \ldots, h_{n-1}, D\right\}$. We denote by $\left\{\Lambda_{0}, \Lambda_{1}, \ldots, \Lambda_{n-1}, \delta\right\}$ the dual basis of $\mathfrak{h}^{*}$, that is,

$$
\left\langle\Lambda_{i}, h_{j}\right\rangle=\delta_{i j}, \quad\left\langle\Lambda_{i}, D\right\rangle=0, \quad\left\langle\delta, h_{i}\right\rangle=0, \quad\langle\delta, D\rangle=1,
$$

and we set $\alpha_{i}=2 \Lambda_{i}-\Lambda_{i-1}-\Lambda_{i+1}+\delta_{i 0} \delta$ for $i=0,1, \ldots, n-1$. In these formulas it is understood that $\Lambda_{n}=\Lambda_{0}$ and $\Lambda_{-1}=\Lambda_{n-1}$. The $n \times n$ matrix $\left[\left\langle\alpha_{i}, h_{j}\right\rangle\right]$ is the generalized Cartan matrix associated to $\widehat{\mathfrak{s l}}_{n}$. The weight lattice is $P=\left(\bigoplus_{i=0}^{n-1} \mathbb{Z} \Lambda_{i}\right) \bigoplus \mathbb{Z} \delta$, its dual is $P^{\vee}=\left(\bigoplus_{i=0}^{n-1} \mathbb{Z} h_{i}\right) \bigoplus \mathbb{Z} D$, and the root lattice is $Q=\bigoplus_{i=0}^{n-1} \mathbb{Z} \alpha_{i}$. One defines $U_{q}\left(\widehat{\mathfrak{s l}}_{n}\right)$ as the associative algebra with 1 over $\mathbb{Q}(q)$ generated by the symbols $e_{i}, f_{i}, 0 \leqq i \leqq n-1$, and $q^{h}, h \in P^{\vee}$, subject to the relations

$$
\begin{gathered}
q^{h} q^{h^{\prime}}=q^{h+h^{\prime}}, \quad q^{0}=1, \\
q^{h} e_{j} q^{-h}=q^{\left\langle\alpha_{j}, h\right\rangle} e_{j}, \\
q^{h} f_{j} q^{-h}=q^{-\left\langle\alpha_{j}, h\right\rangle} f_{j}, \\
{\left[e_{i}, f_{j}\right]=\delta_{i j} \frac{q^{h_{t}}-q^{-h_{i}}}{q-q^{-1}},} \\
\sum_{k=0}^{1-\left\langle\alpha_{i}, h_{j}\right\rangle}(-1)^{k}\left[\begin{array}{c}
1-\left\langle\alpha_{i}, h_{j}\right\rangle \\
k
\end{array}\right] e_{i}^{1-\left\langle\alpha_{i}, h_{j}\right\rangle-k} e_{j} e_{i}^{k}=0 \quad(i \neq j), \\
\sum_{k=0}^{1-\left\langle\alpha_{i}, h_{j}\right\rangle}(-1)^{k}\left[\begin{array}{c}
1-\left\langle\alpha_{i}, h_{j}\right\rangle \\
k
\end{array}\right] f_{i}^{1-\left\langle\alpha_{i}, h_{j}\right\rangle-k} f_{j} f_{i}^{k}=0 \quad(i \neq j) .
\end{gathered}
$$

Here we follow the usual notation for $q$-integers, $q$-factorials and $q$-binomial coefficients:

$$
[k]=\frac{q^{k}-q^{-k}}{q-q^{-1}}, \quad[k] !=[k][k-1] \cdots[1], \quad\left[\begin{array}{c}
m \\
k
\end{array}\right]=\frac{[m] !}{[m-k] ![k] !} .
$$

We now recall some definitions relative to $U_{q}\left(\widehat{\mathfrak{s}}_{n}\right)$-modules. Let $M$ be a $U_{q}\left(\widehat{\mathfrak{s}}_{n}\right)$ module and $\Lambda \in P$ a weight. The subspace

$$
M_{\Lambda}=\left\{v \in M \mid q^{h} v=q^{\langle\Lambda, h\rangle} v, h \in P^{\vee}\right\}
$$


is called the weight space of weight $\Lambda$ of $M$ and its elements are called the weight vectors of weight $\Lambda$. The module $M$ i said to be integrable if

(i) $M=\bigoplus_{\Lambda \in P} M_{\Lambda}$,

(ii) $\operatorname{dim} M_{\Lambda}<\infty$ for $\Lambda \in P$,

(iii) for $i=0,1, \ldots, n-1, M$ decomposes into a direct sum of finite dimensional $U_{i}$-modules,

where $U_{i}$ denotes the subalgebra of $U_{q}\left(\widehat{\mathfrak{s l}}_{n}\right)$ generated by $e_{i}, f_{i}, q^{h_{i}}, q^{-h_{i}}$.

A highest weight vector $v \in M$ is a vector annihilated by all raising operators $e_{i}$. The module $M$ is said to be a highest weight module if there exists a highest weight vector $v$ such that $M=U_{q}\left(\widehat{\mathfrak{s l}}_{n}\right) v$. The weight of $v$ is called the highest weight of $M$.

By the representation theory of $U_{q}\left(\widehat{\mathfrak{s l}}_{n}\right)$, there exists for each dominant integral weight $\Lambda$ (i.e. $\left\langle\Lambda, h_{i}\right\rangle \in \mathbb{Z}_{+}$for $\left.i=0,1, \ldots, n-1\right)$ a unique integrable highest weight module $M(\Lambda)$ with highest weight $\Lambda$.

4.2. The Fock space. An important representation of $U_{q}\left(\widehat{\mathfrak{s l}}_{n}\right)$ can be obtained by letting it act on an infinite dimensional vector space with distinguished basis the set $\mathcal{P}$ of all Young diagrams (or partitions):

$$
\mathcal{F}=\bigoplus_{\lambda \in \mathcal{P}} \mathbb{Q}(q) \lambda
$$

$\mathcal{F}$ is known as the Fock space. To describe this action we need to introduce some notation.

Let $i \in\{0,1, \ldots, n-1\}$ and let $\lambda, v$ be two partitions such that $v$ is obtained from $\lambda$ by filling an indent $i$-node $\gamma$ (see Sect. 2). We set:

$N_{i}(\lambda)=\#\{$ indent $i$-nodes of $\lambda\}-\#\{$ removable $i$-nodes of $\lambda\}$,

$N_{i}^{l}(\lambda, v)=\#\{$ indent $i$-nodes of $\lambda$ situated to the left of $\gamma$ (not counting $\gamma$ ) $\}$

$-\#\{$ removable $i$-nodes of $\lambda$ situated to the left of $\gamma\}$,

$N_{i}^{r}(\lambda, v)=\#\{$ indent $i$-nodes of $\lambda$ situated to the right of $\gamma$ (not counting

$\gamma)\}-\#\{$ removable $i$-nodes of $\lambda$ situated to the right of $\gamma\}$,

$N^{0}(\lambda)=\#\{0$-nodes of $\lambda\}$.

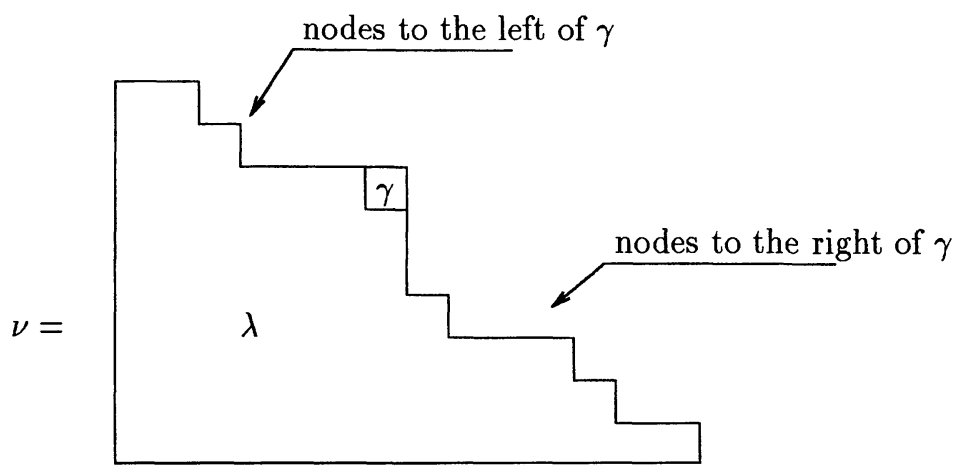

The following result is due to Hayashi [13], and the formulation that we use has been given by Misra and Miwa [36]. 
Theorem 4.1. The algebra $U_{q}\left(\widehat{\mathfrak{s l}}_{n}\right)$ acts on $\mathcal{F}$ by

$$
\begin{aligned}
& q^{h_{i}} \lambda=q^{N_{l}(\lambda)} \lambda, \\
& q^{D} \lambda=q^{-N^{0}(\lambda)} \lambda, \\
& f_{i} \lambda=\sum_{v} q^{N_{i}^{r}(\lambda, v)} v, \text { sum over all partitions } v \text { such that } v / \lambda \text { is a } i \text {-node, } \\
& e_{i} v=\sum_{\lambda} q^{-N_{i}^{l}(\lambda, v)} \lambda \text {, sum over all partitions } \lambda \text { such that } v / \lambda \text { is a } i \text {-node. }
\end{aligned}
$$

It is easy to see that $\mathcal{F}$ is an integrable $U_{q}\left(\widehat{\mathfrak{s l}}_{n}\right)$-module. It is not irreducible. Actually it decomposes as

$$
\mathcal{F} \cong \bigoplus_{k \geqq 0} M\left(\Lambda_{0}-k \delta\right)^{\oplus p(k)}
$$

Obviously, the empty partition $\emptyset$ is a highest weight vector of weight $\Lambda_{0}$. The submodule $U_{q}\left(\widehat{\mathfrak{s l}}_{n}\right) \emptyset$ is isomorphic to $M\left(\Lambda_{0}\right)$, the so-called basic representation of $U_{q}\left(\widehat{\mathfrak{s l}}_{n}\right)$.

There is a natural grading of $\mathcal{F}$ given by

$$
\operatorname{deg} \lambda=m \Longleftrightarrow \lambda \vdash m \text {. }
$$

This is the principal gradation of $\mathcal{F}$ (see [18]). Set

$$
\mathcal{F}_{m}=\bigoplus_{\operatorname{deg} \lambda=m} \mathbb{Q}(q) \lambda, \quad M\left(\Lambda_{0}\right)_{m}=M\left(\Lambda_{0}\right) \cap \mathcal{F}_{m}
$$

Then, $\operatorname{dim} M\left(\Lambda_{0}\right)_{m}=p^{*}(m)$, the number of $n$-regular partitions of $m$.

To conclude, we note that the $U_{q}\left(\widehat{\mathfrak{s l}}_{n}\right)$-modules we have described should be regarded as some $q$-analogues of the Grothendieck rings of the Hecke algebras. More precisely, denoting by $\overline{\mathcal{F}}$ and $\overline{M\left(\Lambda_{0}\right)}$ the $\widehat{\mathfrak{s l}}_{n}$-modules obtained by specializing $q$ to 1 , we see that

$$
\overline{\mathcal{F}} \cong \mathcal{G}(v) \otimes_{\mathbb{Z}} \mathbb{Q}, \quad \overline{M\left(\Lambda_{0}\right)} \cong \mathcal{K}(\zeta) \otimes_{\mathbb{Z}} \mathbb{Q},
$$

via the mapping $\lambda \longrightarrow s_{\lambda}$. The raising operators $e_{i}$ and lowering operators $f_{i}$ of $\widehat{\mathfrak{s l}}_{n}$ are identified in these isomorphisms with the $i$-restricting and $i$-inducing operators. Thus a natural correspondence shows up between the vectors of degree $m$ of the basic representation of $\widehat{\mathfrak{s l}}_{n}$ and the classes of projective modules of $H_{m}(\zeta)$. Using this correspondence, the problem of computing the decomposition matrices of the algebras $H_{m}(\zeta)$ translates into the problem of computing a certain distinguished basis of the basic $\widehat{\mathfrak{s l}}_{n}$-module realized as a submodule of the Fock space.

\section{The Crystal Graphs of $\mathcal{F}$ and $M\left(\Lambda_{0}\right)$}

Canonical bases of the integrable highest weight modules of affine Lie algebras have been introduced by Kashiwara as classical limits of the so-called global crystal bases of the corresponding quantized affine Lie algebras. The first step in determining these bases is to obtain the crystal graph of the module under study. In the case of $\mathcal{F}$ and $M\left(\Lambda_{0}\right)$ the crystal graphs have been first described by Misra and Miwa [36]. We shall briefly recall their results. 
5.1. The crystal basis at $q=0$ of $\mathcal{F}$. Let $A \subset \mathbb{Q}(q)$ denote the subring of rational functions without pole at $q=0$. The lower crystal basis at $q=0$ of $\mathcal{F}$, is the pair $(L, B)$ where $L$ is the lower crystal lattice given by

$$
L=\bigoplus_{\lambda \in \mathcal{P}} A \lambda
$$

and $B$ is the basis of the $\mathbb{Q}$-vector space $L / q L$ given by

$$
B=\{\lambda \bmod q L \mid \lambda \in \mathcal{P}\} .
$$

The main properties of $(L, B)$ are stated in terms of Kashiwara's operators $\tilde{e}_{i}, \tilde{f}_{i}$, the definition of which we shall now recall. These are endomorphisms of $\mathcal{F}$ coming from the decomposition of $\mathcal{F}$ into a direct sum of simple $U_{i}$-modules. The subalgebra $U_{i}$ being isomorphic to $U_{q}\left(\mathfrak{s l}_{2}\right)$, it is known from the representation theory of $U_{q}\left(\mathfrak{s l}_{2}\right)$ that these simple $U_{i}$-modules are of the form

$$
V_{l}=\bigoplus_{k=0}^{l} \mathbb{Q}(q) u_{k}^{(l)}
$$

where

$$
e_{i} u_{0}^{(l)}=f_{i} u_{l}^{(l)}=0 \quad \text { and } \quad u_{k}^{(l)}=\frac{f_{i}^{k}}{[k] !} u_{0}^{(l)} .
$$

By definition $\tilde{e}_{i}$ and $\tilde{f}_{i}$ operate on each $V_{l}$ by

$$
\tilde{f}_{i} u_{k}^{(l)}=u_{k+1}^{(l)}, \quad \tilde{e}_{i} u_{k}^{(l)}=u_{k-1}^{(l)},
$$

where we understand $u_{-1}^{(l)}=u_{l+1}^{(l)}=0$. The endomorphisms $\tilde{e}_{i}, \tilde{f}_{i}$ are then extended to $\mathcal{F}$ using the isomorphism of $U_{i}$-modules $\Phi: \mathcal{F} \cong \bigoplus_{k} V_{l_{k}}$ (one checks that this does not depend on the choice of $\Phi)$. We can now state

Theorem 5.1 (Misra, Miwa). The crystal lattice $L$ is invariant under $\tilde{e}_{i}, \tilde{f}_{i}$, that is,

$$
\tilde{e}_{i} L \subset L, \quad \tilde{f}_{i} L \subset L .
$$

Therefore, $\tilde{e}_{i}, \tilde{f}_{i}$ induce endomorphisms of $L / q L$ (still denoted by $\tilde{e}_{i}, \tilde{f}_{i}$ ) whose action on $B$ is purely combinatorial, namely

(i) $\tilde{e}_{i}(v \bmod q L)=0$ if $v$ has no good $i$-node,

(ii) if $v$ has a good $i$-node and if $\lambda$ is the partition obtained by removing this node from $v$, then $\tilde{e}_{i}(v \bmod q L)=\lambda \bmod q L$,

(iii) $\tilde{f}_{i}(\lambda \bmod q L)=0$ if $\lambda$ has no good indent $i$-node,

(iv) if $\lambda$ has a good indent $i$-node and if $v$ is the partition obtained by adding this node to $\lambda$, then $\tilde{f}_{i}(\lambda \bmod q L)=v \bmod q L$.

Here, we have called good indent $i$-node an indent $i$-node $\gamma$ of $\lambda$ such that if $v$ is obtained by adding $\gamma$ to $\lambda$, then $\gamma$ is a good (removable) node of $v$. Equivalently, the good indent $i$-node of $\lambda$ (if any) is indicated by the last $I$ outside the brackets in the algorithmic description of Sect. 2.

It follows from Theorem 5.1 that the action of $\tilde{e}_{i}, \tilde{f}_{i}$ on $B$ can be recorded on a graph $\Gamma_{n}$ named the crystal graph of $\mathcal{F}$, whose set of vertices is $B$ and whose 
arrows are given by

$$
\begin{aligned}
\lambda \stackrel{i}{\longrightarrow} v & \Longleftrightarrow \tilde{f}_{i}(\lambda \bmod q L)=v \bmod q L \\
& \Longleftrightarrow \lambda \text { is obtained from } v \text { by removing a good } i \text {-node }
\end{aligned}
$$

5.2. The crystal basis at $q=0$ of $M\left(\Lambda_{0}\right)$. There is a unique decomposition of $\mathcal{F}$ into a direct sum of $U_{q}\left(\widehat{\mathfrak{s l}}_{n}\right)$-modules

$$
\mathcal{F} \cong M\left(\Lambda_{0}\right) \oplus\left(\bigoplus_{k \geqq 1} M\left(\Lambda_{0}-k \delta\right)^{\oplus p(k)}\right)
$$

This defines a projection $\Pi: \mathcal{F} \longrightarrow M\left(\Lambda_{0}\right)$ which induces a projection $\Pi: L / q L \longrightarrow$ $L\left(\Lambda_{0}\right) / q L\left(\Lambda_{0}\right)$, where $L\left(\Lambda_{0}\right):=L \cap M\left(\Lambda_{0}\right)$. It turns out that $B\left(\Lambda_{0}\right):=\Pi(B) \backslash\{0\}$ is given by

$$
\left.B\left(\Lambda_{0}\right)=\{\Pi \mu \bmod q L) \mid \mu \in \mathcal{P}_{n}\right\},
$$

where $\mathcal{P}_{n}$ denotes the set of $n$-regular partitions. The pair $\left(B\left(\Lambda_{0}\right), L\left(\Lambda_{0}\right)\right)$ satisfies the same properties as those of $(L, B)$ stated in Theorem 5.1. The crystal graph of $M\left(\Lambda_{0}\right)$ is none other than the connected component of $\Gamma_{n}$ containing the empty partition $\emptyset$. Thus, comparing with Theorem 2.3, Definition 2.4, we have obtained

Proposition 5.2. When $n=p$ is a prime, the crystal graph of $M\left(\Lambda_{0}\right)$ coincides with Kleshchev's p-good lattice.

5.3. Reduced n-quotient and a bijective proof of a classical formula. We conclude this section by discussing a classical combinatorial problem arising in the $p$-modular representation theory of $\mathfrak{\Xi}_{m}$ (or similarly in the representation theory of $H_{m}(\zeta)$ ) and in the study of the basic $\widehat{\mathfrak{s l}}_{n}$-module $\overline{M\left(\Lambda_{0}\right)}$. Denote by $\lambda_{(n)}$ and $\lambda^{(n)}=\left(\lambda^{0}, \ldots, \lambda^{n-1}\right)$ the $n$-core and $n$-quotient of a partition $\lambda$ (see [15]). The integer

$$
|\lambda|_{n}:=\sum_{i=0}^{n-1} \operatorname{deg} \lambda^{i}=\frac{1}{n}\left(\operatorname{deg} \lambda-\operatorname{deg} \lambda_{(n)}\right)
$$

is called the $n$-weight of $\lambda$.

Problem 5.3. What is the number $N_{n m}(\theta)$ of $n$-regular partitions of $m$ with $n$-core $\theta$ ?

According to the Nakayama conjecture for $\Xi_{m}$ (or its analogue for $H_{m}(\zeta)$ ), the $p$-cores of the partitions $\lambda$ of $m$ parametrize the blocks of $\mathbb{F}_{p} \widetilde{\Xi}_{m}$ (resp. the $n$-cores parametrize the blocks of $\left.H_{m}(\zeta)\right)$.

On the other hand, the set of weights of $\overline{M\left(\Lambda_{0}\right)}$ (and of $\overline{\mathcal{F}}$ ) is

$$
P\left(\Lambda_{0}\right)=\left\{w \Lambda_{0}-k \delta \mid w \in W, k \in \mathbb{N}\right\},
$$

where $W$ denotes the Weyl group of $\widehat{\mathfrak{s l}}_{n}$ (see [18]). A partition $\lambda$, when regarded as a weight vector of $\overline{\mathcal{F}}$, has a weight of the form wt $(\mu)=w \Lambda_{0}-k \delta$, where $k=|\lambda|_{n}$.

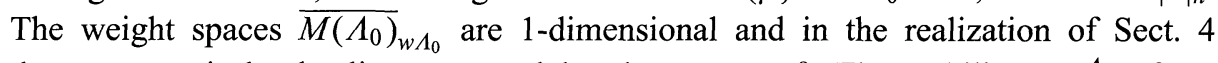
they are precisely the lines spanned by the $n$-cores $\theta$. The stabilizer $W^{\Lambda_{0}}$ of $\Lambda_{0}$ in $W$ is the subgroup generated by the reflections $s_{1}, s_{2}, \ldots s_{n-1}$ associated with the 
simple roots $\alpha_{1}, \alpha_{2}, \ldots, \alpha_{n-1}$. We shall denote by $\dot{w}_{\theta}$ the unique element in $W / W^{\Lambda_{0}}$ such that wt $(\theta)=\dot{w}_{\theta} \Lambda_{0}$, and by $w_{\theta}$ a representative of $\dot{w}_{\theta}$.

The number $N_{n m}(\theta)$ may therefore be interpreted in different ways. It is

(i) the dimension of the block of $H_{m}(\zeta)$ parametrized by $\theta$,

(ii) if $n=p$ is a prime, the dimension of the block of $\mathbb{F}_{p} \Im_{m}$ parametrized by $\theta$,

(iii) the dimension of the weight space of $\overline{M\left(\Lambda_{0}\right)}$ with weight $w_{\theta} \Lambda_{0}-s \delta$, where $s=(m-\operatorname{deg} \theta) / n$.

In the fifties Robinson proved the formula

$$
N_{n m}(\theta)=\sum_{i_{1}+i_{2}+} \sum_{+i_{n-1}=s} p\left(i_{1}\right) p\left(i_{2}\right) \cdots p\left(i_{n-1}\right)
$$

which shows that this number does not depend on $\theta$ but only on $s$ (see [39], p. 112). This is clear from the interpretation (iii) since the character of $\overline{M\left(\Lambda_{0}\right)}$ must be invariant under the action of $W$.

We would like to point out that one can use the crystal graph of $M\left(\Lambda_{0}\right)$ to obtain a bijective proof of this result. The basic idea is that the set of vertices of the crystal graph of a $U_{q}(\mathfrak{g})$-module is endowed with a natural action of the Weyl group of the Lie algebra $\mathfrak{g}$ [21] (see [29] for an application of this action in the case $\left.\mathfrak{g}=\mathfrak{s l}_{n}\right)$.

From now on, we shall identify the vector $\mu \bmod q L\left(\Lambda_{0}\right)$ of $B\left(\Lambda_{0}\right)$ with the corresponding $n$-regular partition $\mu$. The action of a simple reflection $s_{i}$ of $W$ on $\mu$ is defined by

$$
s_{i} \mu=\left\{\begin{array}{cl}
\tilde{f}_{i}^{\left\langle\mathrm{wt}(\mu), h_{i}\right\rangle} \mu & \text { if }\left\langle\mathrm{wt}(\mu), h_{i}\right\rangle \geqq 0 \\
\tilde{e}_{i}^{-\left\langle\mathrm{wt}(\mu), h_{i}\right\rangle} \mu & \text { if }\left\langle\mathrm{wt}(\mu), h_{i}\right\rangle \leqq 0
\end{array} .\right.
$$

In other words, $s_{i} \mu$ is the mirror image of $\mu$ in the reflection through the middle of the string of colour $i$ of the crystal graph going through $\mu$.

Lemma 5.4. This action is a lifting to $B\left(\Lambda_{0}\right)$ of the usual action of $W$ on $P\left(\Lambda_{0}\right)$, in the sense that

$$
\mathrm{wt}\left(s_{i} \mu\right)=s_{i}(\mathrm{wt}(\mu)) .
$$

Moreover the stabilizers of $\mu$ and $\mathrm{wt}(\mu)$ coincide:

$$
w \mu=\mu \Longleftrightarrow w(\operatorname{wt}(\mu))=\operatorname{wt}(\mu) \text {. }
$$

Proof. One has wt $\left(\tilde{f}_{i} \mu\right)=\operatorname{wt}(\mu)-\alpha_{i}$ and $\operatorname{wt}\left(\tilde{e}_{i} \mu\right)=\operatorname{wt}(\mu)+\alpha_{i}$. Therefore wt $\left(s_{i} \mu\right)$ $=\mathrm{wt}(\mu)-\left\langle\mathrm{wt}(\mu), h_{i}\right\rangle \alpha_{i}=s_{i}(\mathrm{wt}(\mu))$. To prove the second fact we can assume that wt $(\mu)$ is dominant, since each $W$-orbit in $P\left(\Lambda_{0}\right)$ contains a dominant weight. Thus, we have wt $(\mu)=\Lambda_{0}-k \delta$ for some integer $k$, so that $\operatorname{Stab}(w t(\mu))=\operatorname{Stab}\left(\Lambda_{0}\right)=$ $W^{\Lambda_{0}}$ (because $w \delta=\delta$ for all $w \in W$ ). Now for $i=1,2, \ldots, n-1,\left\langle\operatorname{wt}(\mu), h_{i}\right\rangle=0$ which shows that $s_{i} \mu=\mu$. Hence $w \mu=\mu$ for $w \in W^{\Lambda_{0}}$.

It follows that the orbits of the action of $W$ on $B\left(\Lambda_{0}\right)$ are all isomorphic to $W / W^{\Lambda_{0}}$, and that they are parametrized by their unique vector of dominant weight, that is, by their unique partition $\mu$ without $n$-core. Therefore for any pair $\theta, \theta^{\dagger}$ of $n$-cores, the map

$$
\mathcal{B}_{\theta \theta^{\dagger}}: \mu \longrightarrow w_{\theta^{\dagger}} w_{\theta}^{-1}(\mu)
$$


establishes a bijection (independent of the representatives $w_{\theta}, w_{\theta^{\dagger}}$ of $\dot{w}_{\theta}, \dot{w}_{\theta^{\dagger}}$ ) between the set of $n$-regular partitions with $n$-core $\theta$ and the set of $n$-regular partitions with $n$-core $\theta^{\dagger}$, which preserves the $n$-weight.

Consider now the family of $n$-cores

$$
\theta_{k}=\left(k^{n-1}(k-1)^{n-1} \cdots 2^{n-1} 1^{n-1}\right), \quad k \geqq 1 .
$$

We have the following lemma, which is a particular case of [39], 6.36, p. 112.

Lemma 5.5. Let $s$ be an integer $\leqq k+1$ and set $m=\operatorname{deg} \theta_{k}+n s$. A partition $\mu$ with $n$-weight $s$ and $n$-core $\theta_{k}$ is $n$-regular iff the $r^{\text {th }}$ constituent of the $n^{\text {th }}$ quotient of $\mu$ is empty, where $r=k \bmod n$.

It is easy to check that if $\mu$ satisfies the conditions of the lemma and $\mu^{\dagger}=$ $\mathcal{B}_{\theta_{k} \theta_{k+1}}(\mu)$, then the $n$-quotient of $\mu^{\dagger}$ is obtained from that of $\mu$ by performing a circular permutation: $\mu^{(j)}=\mu^{\dagger(j+1)}, j \in \mathbb{Z} / n \mathbb{Z}$.

Now, let $\mu$ be a $n$-regular partition with $n$-weight $s$, and set $\mu^{\dagger}=\mathcal{B}_{\mu_{(n)} \theta_{k+1}}(\mu)$ for $k \geqq s-1$. Then, the map

$$
\mu \longrightarrow\left(\mu_{(n)} ; \mu^{\dagger(k+1)}, \ldots, \mu^{\dagger(k+n-1)}\right)
$$

is well-defined (i.e. does not depend on the choice of $k$ ). We call the $(n-1)$-uple $\left(\mu^{\dagger(k+1)}, \ldots, \mu^{\dagger(k+n-1)}\right)$ the reduced $n$-quotient of $\mu$. The above arguments show that a $n$-regular partition is uniquely determined by its $n$-core and its reduced $n$-quotient, which proves the formula of Robinson.

Example 5.6. We illustrate these definitions by computing the reduced 3-quotients of the 5 partitions of $m=6$ with empty 3 -core.

$\begin{array}{llll}\mu & \mu^{\dagger}(k=1) & \text { 3-quotient of } \mu^{\dagger} & \text { reduced 3-quotient } \\ (6) & (71) & ((2), \emptyset, \emptyset) & (\emptyset,(2)) \\ \left(41^{2}\right) & \left(42^{2}\right) & \left(\left(1^{2}\right), \emptyset, \emptyset\right) & \left(\emptyset,\left(1^{2}\right)\right) \\ (321) & \left(32^{2} 1\right) & \left(\emptyset, \emptyset,\left(1^{2}\right)\right) & \left(\left(1^{2}\right), \emptyset\right) \\ (51) & (62) & (\emptyset, \emptyset,(2)) & ((2), \emptyset) \\ \left(3^{2}\right) & \left(4^{2}\right) & ((1), \emptyset,(1)) & ((1),(1))\end{array}$

\section{The Lower Global Crystal Basis of $M\left(\Lambda_{0}\right)$}

We recall the definition of Kashiwara's lower global crystal basis and present a simple and effective algorithm to compute it. This algorithm is inspired by the methods described in [15] for finding the decomposition matrices of the symmetric groups. Then we state our main conjecture.

6.1. The definition of $\{G(\mu)\}$. We need to introduce an involution $v \longrightarrow \bar{v}$ of $M\left(\Lambda_{0}\right)$. We start from the involution $P \longrightarrow \bar{P}$ of $U_{q}\left(\widehat{\mathfrak{s l}}_{n}\right)$ defined as the ring 
automorphism satisfying

$$
\begin{gathered}
\bar{q}=q^{-1}, \quad \overline{q^{h}}=q^{-h}, \quad(h \in \mathfrak{h}), \\
\overline{e_{i}}=e_{i}, \quad \overline{f_{i}}=f_{i}, \quad i=0,1, \ldots, n-1 .
\end{gathered}
$$

Then, for $v=P \emptyset \in M\left(\Lambda_{0}\right)$, we set $\bar{v}=\bar{P} \emptyset$. Finally, we denote by $U_{\mathbb{Q}}^{-}$the sub$\mathbb{Q}\left[q, q^{-1}\right]$-algebra of $U_{q}\left(\widehat{\mathfrak{s l}}_{n}\right)$ generated by $f_{i}^{(k)}:=f_{i}^{k} /[k]$ !, and we set $M\left(\Lambda_{0}\right)_{\mathbb{Q}}=$ $U_{\mathbb{Q}}^{-} \emptyset$. We can now state

Theorem 6.1 (Kashiwara [19]). There exists a unique $\mathbb{Q}\left[q, q^{-1}\right]$-basis $\{G(\mu), \mu \in$ $\left.\mathcal{P}_{n}\right\}$ of $M\left(\Lambda_{0}\right)_{\mathbb{Q}}$ such that

(G1) $G(\mu) \equiv \mu \bmod q L$,

(G2) $\overline{G(\mu)}=G(\mu)$.

The basis $\{G(\mu)\}$ is called the lower global crystal basis of $M\left(\Lambda_{0}\right)_{\mathbb{Q}}\left(\right.$ or $\left.M\left(\Lambda_{0}\right)\right)$.

6.2. The algorithm. Let $m$ be a fixed integer. We want to compute the finite subset of $\{G(\mu)\}$ consisting of the vectors of degree $m$. More precisely we want to determine the expansion

$$
G(\mu)=\sum_{\lambda \vdash m} d_{\lambda \mu}(q) \lambda
$$

of these $G(\mu)$ on the natural basis $\{\lambda\}$ of $\mathcal{F}$. The matrix $\mathbf{D}_{m}(q)=\left[d_{\lambda \mu}(q)\right]$ of the coefficients of this expansion is a rectangular matrix whose rows are labelled by the partitions $\lambda$ of $m$ and whose columns are labelled by the $n$-regular partitions $\mu$ of $m$.

Lemma 6.2. Let $v \in M\left(\Lambda_{0}\right)$ be a vector of the type

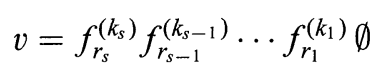

for some positive integers $k_{1}, \ldots, k_{s}$, and some sequence of elements $\left(r_{1}, \ldots, r_{s}\right)$ of $\{0,1, \ldots, n-1\}$. Then the coordinates of $v$ on the basis $\{\lambda\}$ of $\mathcal{F}$ belong to $\mathbb{N}\left[q, q^{-1}\right]$.

Proof. By induction on $s$, it is enough to prove that for $\lambda, \tau \in \mathcal{P}$, the coefficient of $\lambda$ in $f_{r_{s}}^{k_{s}} \tau$ is divisible by [ $\left.k_{s}\right]$ !. If this coefficient is nonzero, then $\lambda$ is obtained from $\tau$ by filling $k_{s}$ indent $r_{s}$-nodes. To $\tau$ and $\lambda$ we associate, as in Sect. 2, two sequences $\mathcal{S}_{\tau}$ and $\mathcal{S}_{v}$ of letters $R$ and $I$ indicating the successive removable and indent $r_{s}$-nodes. We write

$$
\mathcal{S}_{\tau}=a_{q} a_{q-1} \cdots a_{1}, \quad \mathcal{S}_{\lambda}=b_{q} b_{q-1} \cdots b_{1}
$$

where $a_{i}, b_{i} \in\{R, I\}$ and $a_{i}=b_{i}$ except for $k_{s}$ letters $a_{j}=I$ for which $b_{j}=R$. Let $J=\left\{j_{1}<\cdots<j_{k}\right\}$ be the set of indices $j$ such that $a_{j} \neq b_{j}$. It follows from the combinatorial description of the action of $f_{i}$ on Young diagrams that $\lambda$ will occur exactly $k_{s}$ ! times in $f_{r_{s}}^{k_{s}} \tau$, corresponding to the $k_{s}$ ! different orders in which we can fill the $k_{s}$ indent nodes. Let $\left(j_{\sigma(1)}, \ldots, j_{\sigma\left(k_{s}\right)}\right)$ be a permutation of the indices of $J$. One checks that the term in $f_{r_{s}}^{k_{s}} \tau$ corresponding to the addition of a node first in 
position $j_{\sigma(1)}$, then in position $j_{\sigma(2)}$, and so on, is equal to $q^{N(\sigma)} \lambda$ where,

$$
N(\sigma)=N(\text { id })+2 \ell(\sigma) .
$$

Here $\ell(\sigma)$ denotes the length of the permutation $\sigma$. Hence the coefficient of $\lambda$ in $f_{r_{s}}^{k_{s}} \tau$ is equal to

$$
\sum_{\sigma} q^{N(\mathrm{id})+2 \ell(\sigma)}=q^{N(\mathrm{id})+\left(\begin{array}{c}
k_{s} \\
2
\end{array}\right)}\left[k_{s}\right] !,
$$

which proves the lemma.

Corollary 6.3. We have $d_{\lambda \mu}(q) \in \mathbb{Q}[q]$.

Proof. Since $G(\mu) \in M\left(\Lambda_{0}\right)_{\mathbb{Q}}$, it is a $\mathbb{Q}\left[q, q^{-1}\right]$-linear combination of vectors of the form (7). Hence by Lemma 6.2, the coefficients $d_{\lambda \mu}(q)$ belong to $\mathbb{Q}\left[q, q^{-1}\right]$. Now it follows from (G1) that $d_{\lambda \mu}(q)$ is regular at $q=0$, which forces $d_{\lambda \mu}(q) \in \mathbb{Q}[q]$.

We can now describe our algorithm for computing $\mathbf{D}_{m}(q)$. Our method is very similar to the techniques explained in [15] for finding the decomposition matrix of the symmetric group $\mathfrak{\Xi}_{m}$ modulo $n$ (in case $n=p$ is a prime). We shall first obtain a "first approximation" $\{A(\mu)\}$ of $\{G(\mu)\}$ which satisfies

(A0) $\left\{A(\mu), \mu \in \mathcal{P}_{n}, \mu \vdash m\right\}$ is a $\mathbb{Q}\left[q, q^{-1}\right]$-basis of $M\left(\Lambda_{0}\right)_{m} \cap M\left(\Lambda_{0}\right)_{\mathbb{Q}}$,

(A1) $A(\mu)=\sum_{\lambda} \alpha_{\lambda \mu}(q) \lambda$, where $\alpha_{\lambda \mu}(q)=0$ unless $\lambda \unlhd \mu, \alpha_{\mu \mu}(q)=1$, and $\alpha_{\lambda \mu}(q) \in \mathbb{N}\left[q, q^{-1}\right]$,

(A2) $\overline{A(\mu)}=A(\mu)$.

We explain the construction of $\{A(\mu)\}$. The vector $A(\mu)$ will be of the type

$$
A(\mu)=f_{r_{s}}^{\left(k_{s}\right)} f_{r_{s-1}}^{\left(k_{s-1}\right)} \cdots f_{r_{1}}^{\left(k_{1}\right)} \emptyset \text {. }
$$

It is clear that $\overline{f_{i}^{(k)}}=f_{i}^{(k)}$, so that (A2) will be automatically fulfilled. Therefore it remains to explain how to get from $\mu$ the two sequences of integers $\left(r_{1}, \ldots, r_{s}\right)$, $\left(k_{1}, \ldots, k_{s}\right)$ such that (A0) (A1) hold true.

For this we shall follow the "ladder method" of [15], 6.3.51, p. 283. We need some terminology. Consider a Young diagram $\mu$ whose boxes are filled with their $n$-residues. For example, if $n=4$,

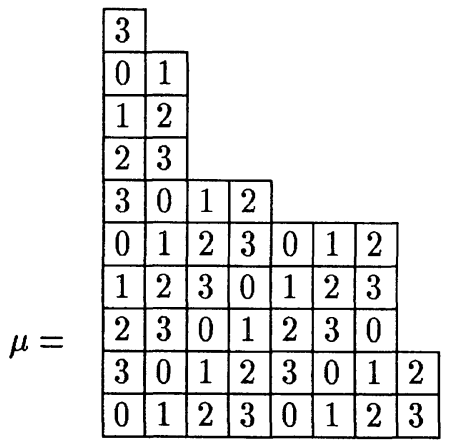

The ladders are the straight lines of equation $y=(1-n) x+k, k=0,1,2, \ldots$ Here we take the origin of coordinates to be the center of the leftmost bottom box of $\mu$. 
Replacing each box by the corresponding node lying at its center we get a picture of the following type (we have drawn only a few of the ladders intersecting $\mu$ ):

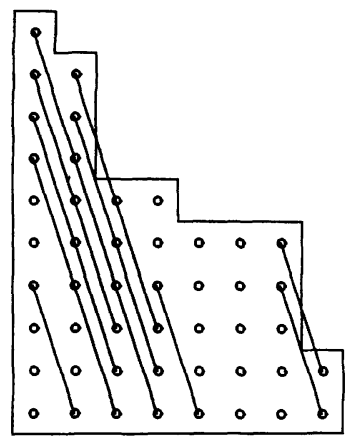

By construction, the nodes of $\mu$ lying on a given ladder $y=(1-n) x+k$ all have the same residue $n-k \bmod n$. Let $s$ be the number of ladders containing at least one node of $\mu$. Denote these ladders from left to right by $L_{1}, \ldots, L_{s}$, so that $L_{1}$ is the ladder through the origin and $L_{s}$ is the rightmost ladder intersecting $\mu$. Let $k_{i}$ be the number of nodes of $\mu$ lying on $L_{i}$, and let $r_{i}$ be their common $n$-residue. Thus in our example $s=23$, and for instance $r_{23}=2, k_{23}=2, r_{22}=3, k_{22}=2, r_{21}=0$, $k_{21}=1$. We can now define, for a $n$-regular partition $\mu$, the vector $A(\mu)$ by formula (8).

Lemma 6.4. The vectors $A(\mu)$ satisfy properties (A0), (A1), (A2) above.

Proof. As already mentioned, (A2) is clear from the definition. Let us prove (A1). This is done by induction on $s$. Let us assume that $A(v)$ satisfies (A1), where $v$ is the partition obtained from $\mu$ by removing the nodes lying on the rightmost ladder $L_{s}$. Let $\tau$ be a partition occurring in the expansion of $A(v)$. Let $\lambda$ be a partition obtained from $\tau$ by filling $k_{s}$ indent $r_{s}$-nodes (if possible). Taking into account Lemma 6.2, all we have to prove is that

(i) $\lambda \unlhd \mu$,

(ii) if $\lambda=\mu$ then $\tau=v$, and the coefficient of $\mu$ in $f_{r_{s}}^{k_{s}} v$ is equal to $\left[k_{s}\right]$ !

The proof of (i) comes from the fact that since $\mu$ is $n$-regular, the nodes of $\mu / v$ lie in the $k_{s}$ lowest positions on the ladder $L_{s}$. We refer to [15], 6.3 .54 for details.

Now if $\lambda=\mu$, then $\tau$ is obtained from $\mu$ by removing $k_{s}$ nodes of residue $r_{s}$. Since we have $\tau \unlhd v$ by induction, the only possibility is $\tau=v$. One computes easily in this case $N($ id $)=-\left(\begin{array}{c}k_{s} \\ 2\end{array}\right)$ (we have used the notation of the proof of Lemma 6.2). Hence the coefficient of $\mu$ in $f_{r_{s}}^{k_{s}} v$ is $\left[k_{s}\right]$ !, which proves (ii).

We now come to the proof of (A0). It follows from (A1) that the vectors $A(\mu)$ are linearly independent. It is also clear that $A(\mu)$ is a vector of degree $m=\operatorname{deg} \mu$ of $M\left(\Lambda_{0}\right)$. The dimension of $M\left(\Lambda_{0}\right)_{m}$ being equal to the number of $n$-regular partitions of $m$, we see that $\{A(\mu)\}$ is a $\mathbb{Q}(q)$-basis of $M\left(\Lambda_{0}\right)$. Write

$$
G(\mu)=\sum_{v} \beta_{v \mu}(q) A(v) .
$$


Then $\beta_{v \mu}\left(q^{-1}\right)=\beta_{v \mu}(q)$ because of (G2) and (A2). Define the following square matrices indexed by $n$-regular partitions of $m$ in decreasing lexicographic order

$$
\Delta=\left[d_{v \mu}(q)\right], \quad B=\left[\beta_{v \mu}(q)\right], \quad A=\left[\alpha_{v \mu}(q)\right] .
$$

Then $A$ is unitriangular with entries in $\mathbb{N}\left[q, q^{-1}\right]$ by (A1), and the entries of $\Delta$ belong to $\mathbb{Q}[q]$ by Corollary 6.3. Therefore the entries of $B=A^{-1} \Delta$ belong to $\mathbb{Q}\left[q, q^{-1}\right]$. Hence $\{A(\mu)\}$ is a $\mathbb{Q}\left[q, q^{-1}\right]$-basis of $M\left(\Lambda_{0}\right)_{\mathbb{Q}}$.

We note that Lakshmibai has constructed in [26], for the general case of a highest weight module over a quantum Kac-Moody algebra, a $\mathbb{Q}\left[q, q^{-1}\right]$-basis consisting of vectors of the form (7). It would be interesting to compare this basis with the basis $\{A(\mu)\}$.

Example 6.5. The basis $\{A(\mu)\}$ of $M\left(\Lambda_{0}\right)_{5}$ for $U_{q}\left(\widehat{\mathfrak{s l}}_{2}\right)$ is given by

$$
\left\{\begin{array}{l}
A(5)=f_{0} f_{1} f_{0} f_{1} f_{0} \emptyset=(5)+(32)+2 q\left(31^{2}\right)+q^{2}\left(2^{2} 1\right)+q^{2}\left(1^{5}\right) \\
A(41)=f_{1} f_{0} f_{1}^{(2)} f_{0} \emptyset=(41)+q\left(21^{3}\right) \\
A(32)=f_{0}^{(2)} f_{1}^{(2)} f_{0} \emptyset=(32)+q\left(31^{2}\right)+q^{2}\left(2^{2} 1\right) .
\end{array}\right.
$$

We now turn to the second step of the algorithm, namely, the computation of $\{G(\mu)\}$ out of $\{A(\mu)\}$.

Lemma 6.6. The coefficients $\beta_{v \mu}(q)$ in (9) are such that $\beta_{v \mu}(q)=0$ unless $v \leqq \mu$, and $\beta_{\mu \mu}(q)=1$.

Proof. Let $\mu$ be a fixed $n$-regular partition of $m$ and let $\tau$ be the greatest partition in the lexicographic order such that $\beta_{\tau \mu}(q) \neq 0$. Then the only nonzero summand in $d_{\tau \mu}(q)=\sum_{v} \alpha_{\tau v}(q) \beta_{v \mu}(q)$ is obtained for $v=\tau$. Indeed, $\alpha_{\tau v}(q)=0$ for $\tau>v$ and $\beta_{v \mu}(q)=0$ for $v>\tau$. Thus, $\beta_{\tau \mu}(q)=d_{\tau \mu}(q)$. Now, since $\beta_{\tau \mu}\left(q^{-1}\right)=\beta_{\tau \mu}(q)$ and $d_{\tau \mu}(q)$ is a polynomial in $q$, we have $\beta_{\tau \mu}(q)=\beta_{\tau \mu}(0)=d_{\tau \mu}(0)$. If $\tau \neq \mu$, then $d_{\tau \mu}(0)=0$ which contradicts the definition of $\tau$. Therefore, $\tau=\mu$ and $\beta_{\mu \mu}(q)=$ $d_{\mu \mu}(0)=1$.

It follows that the basis $\{G(\mu)\}$ can easily be obtained by a "triangular" algorithm. Indeed, let

$$
\mu^{(1)}=(m)>\mu^{(2)}=(m-1,1)>\cdots>\mu^{(t)}
$$

be the list of $n$-regular partitions of $m$ sorted in lexicographic order. By Lemma 6.6, $G\left(\mu^{(t)}\right)=A\left(\mu^{(t)}\right)$. Now suppose by induction that we have computed the expansion on the basis $\{\lambda\}$ of the vectors

$$
G\left(\mu^{(i+1)}\right), G\left(\mu^{(i+2)}\right), \ldots, G\left(\mu^{(t)}\right) .
$$

Then

$$
G\left(\mu^{(i)}\right)=A\left(\mu^{(i)}\right)-\gamma_{i+1}(q) G\left(\mu^{(i+1)}\right)-\cdots-\gamma_{t}(q) G\left(\mu^{(t)}\right),
$$

where the $\gamma_{s}(q)$ are completely determined by

$$
\gamma_{s}\left(q^{-1}\right)=\gamma_{s}(q) \text { and } G\left(\mu^{(i)}\right) \equiv \mu^{(i)} \bmod q L .
$$


Indeed, if $\alpha_{\mu^{(i+1)} \mu^{(\imath)}}(q)=\sum_{j=-r}^{s} a_{j} q^{j}$, then $\gamma_{i+1}(q)=\sum_{j=-r}^{0} a_{j} q^{j}+\sum_{j=1}^{r} a_{-j} q^{j}$. Next, if the coefficient of $\mu^{(i+2)}$ in $A\left(\mu^{(i)}\right)-\gamma_{i+1}(q) G\left(\mu^{(i+1)}\right)$ is $\sum_{j=-t}^{u} b_{J} q^{j}$, then $\gamma_{i+2}(q)=$ $\sum_{j=-t}^{0} b_{j} q^{j}+\sum_{j=1}^{t} b_{-j} q^{j}$. And so on. Finally, we note that it follows from (A1) that $\gamma_{s}(q)$ has integer coefficients and is zero unless $\mu^{(s)} \unlhd \mu^{(i)}$.

Example 67 . We continue the previous example. We have $\mu^{(1)}=(5), \mu^{(2)}=(41)$, $\mu^{(3)}=(32)$. We see that $A(32) \equiv(32) \bmod q L$ and $A(41) \equiv(41) \bmod q L$, so that $G(32)=A(32)$ and $G(41)=A(41)$. Next,

$$
G(5)=A(5)-\gamma_{2}(q) A(41)-\gamma_{3}(q) A(32)
$$

Since (41) does not appear in $A(5), \gamma_{2}(q)=0$. Finally, since (32) occurs with coefficient 1 , we have $\gamma_{3}(q)=1$ and

$$
G(5)=A(5)-G(32)=(5)+q\left(31^{2}\right)+q^{2}\left(1^{5}\right) .
$$

From the description of the algorithm we deduce immediately the following

Theorem 6.8. The coefficients $d_{\lambda \mu}(q)$ in the expansion

$$
G(\mu)=\sum_{\lambda} d_{\lambda \mu}(q) \lambda
$$

have the following properties:

(i) $d_{\lambda \mu}(q) \in \mathbb{Z}[q]$,

(ii) $d_{\mu \mu}(q)=1$ and for $\lambda \neq \mu, d_{\lambda \mu}(0)=0$,

(iii) $d_{\lambda \mu}(q)=0$ unless $\lambda$ and $\mu$ have the same degree, the same $n$-core, and $\lambda \unlhd \mu$.

Proof. The only point that has not been discussed yet is the fact that $d_{\lambda \mu}(q)=0$ unless $\lambda$ and $\mu$ have the same $n$-core. This amounts to saying that $G(\mu)$ is a weight vector of $M\left(\Lambda_{0}\right)$, which is clear from the construction.

Comparing (ii) (iii) with the properties noted above for the decomposition numbers $d_{\lambda \mu}$ of the Hecke algebra $H_{m}(\zeta)$, we are led to our main

Conjecture 6.9. For $\lambda \in \mathcal{P}, \mu \in \mathcal{P}_{n}$ partitions of $m$, one has

(i) $d_{\lambda \mu}(q) \in \mathbb{N}[q]$,

(ii) $d_{\lambda_{\mu}}(1)=d_{\lambda \mu}$.

This conjecture agrees with the decomposition matrices computed by James for $m \leqq 10$ [14]. The case $n=2,(\zeta=-1)$ has been further considered by James and Mathas [16] who obtained in this case the decomposition matrices up to $m=20$. We have also checked our conjecture on these matrices. 


\section{The Basis $\{G(\mu)\}$ and the Mullineux-Kleshchev Involution}

In this section we prove a symmetry property of the basis $\{G(\mu)\}$ related to another classical combinatorial problem of the modular representation theory of the symmetric groups.

Given an irreducible $\mathfrak{\Xi}_{m}$-module $D(\mu)$ over a field of characteristic $p$, one can form its tensor product with the one-dimensional sign representation of $\mathfrak{\Xi}_{m}$, which is another simple module $D\left(\mu^{*}\right)$. The problem is to describe in combinatorial terms the involution $\mu \mapsto \mu^{*}$. In characteristic 0 , it is well known that $\mu^{*}$ coincides with the conjugate partition $\mu^{\prime}$ of $\mu$. The answer in the characteristic $p$ case is more difficult.

In 1979, Mullineux constructed an involution $\mu \mapsto \mu^{\vee}$ on the set of $p$-regular partitions and conjectured that $\mu^{*}=\mu^{\vee}$ [37]. There have been several papers on this subject giving more and more evidence that Mullineux's conjecture was true (e.g. Martin [34, 35], Andrews and Olsson [1], Bessenrodt and Olsson [3]), but the proof has been obtained only recently by Ford and Kleshchev [12], using the techniques developed by Kleshchev in [25]. In fact, Kleshchev first gave the following description of $\mu^{*}$ :

Theorem 7.1 (Kleshchev [25]). Let $\mu$ be a p-regular partition and let

$$
\emptyset \stackrel{r_{1}^{k_{1}}}{\longrightarrow} \cdot \stackrel{r_{2}^{k_{2}}}{\longrightarrow} \cdots \stackrel{r_{s}^{k_{s}}}{\longrightarrow} \mu
$$

be a path from $\emptyset$ to $\mu$ in Kleshchev's p-good lattice. (The notation means that one first goes through $k_{1}$ arrows labelled $r_{1}$, then $k_{2}$ arrows labelled $r_{2}$, and so on. ) Then, the path

$$
\emptyset \stackrel{\left(-r_{1}\right)^{k_{1}}}{\longrightarrow} \cdot \stackrel{\left(-r_{2}\right)^{k_{2}}}{\longrightarrow} \cdots \stackrel{\left(-r_{s}\right)^{k_{s}}}{\longrightarrow} \mu^{*}
$$

connects $\emptyset$ to $\mu^{*}$. (Here the labels $\left(-r_{i}\right)$ are understood modulo $p$.)

After that, Ford and Kleshchev proved that this combinatorial description was equivalent to the very different description of $\mu^{\vee}$ by Mullineux. We shall therefore call the map $\mu \mapsto \mu^{*}$ the Mullineux-Kleshchev map.

The Mullineux-Kleshchev map is associated with a natural symmetry of the decomposition matrices of the symmetric groups. Indeed, denoting by $M^{\dagger}$ the dual of an $\mathfrak{\Xi}_{m}$-module $M$, we have

$$
\begin{aligned}
d_{\lambda \mu} & =[S(\lambda): D(\mu)]=[S(\lambda) \otimes \operatorname{sgn}: D(\mu) \otimes \operatorname{sgn}]=\left[S\left(\lambda^{\prime}\right)^{\dagger}: D\left(\mu^{*}\right)\right] \\
& =\left[S\left(\lambda^{\prime}\right): D\left(\mu^{*}\right)\right]=d_{\lambda^{\prime} \mu^{*}} .
\end{aligned}
$$

We shall now prove an analog of this relation for the coefficients $d_{\lambda \mu}(q)$ of the global crystal basis $\{G(\mu)\}$. We first remark that the primality of $p$ is never used in the combinatorial descriptions of the Mullineux-Kleshchev map. In other words, we can define using the crystal graph $\Gamma\left(\Lambda_{0}\right)$ of $M\left(\Lambda_{0}\right)$ an involution still denoted by $\mu \mapsto \mu^{*}$ on the set of $n$-regular partitions by the following rule:

$$
\begin{aligned}
& \text { if } \emptyset \stackrel{r_{1}^{k_{1}}}{\longrightarrow} \cdot \stackrel{r_{2}^{k_{2}}}{\longrightarrow} \cdots \stackrel{r_{s}^{k_{s}}}{\longrightarrow} \mu \text { is a path from } \emptyset \text { to } \mu \text { in } \Gamma\left(\Lambda_{0}\right) \text {, then } \\
& \emptyset \stackrel{\left(-r_{1}\right)^{k_{1}}}{\longrightarrow} \cdot \stackrel{\left(-r_{2}\right)^{k_{2}}}{\longrightarrow} \cdots \stackrel{\left(-r_{s}\right)^{k_{s}}}{\longrightarrow} \mu \text { is a path from } \emptyset \text { to } \mu^{*} .
\end{aligned}
$$


With this definition, we have

Theorem 7.2. For $\lambda \in \mathcal{P}, \mu \in \mathcal{P}_{n}$, there holds

$$
d_{\lambda^{\prime} \mu^{*}}(q)=q^{|\mu|_{n}} d_{\lambda \mu}\left(q^{-1}\right)
$$

where $|\mu|_{n}$ denotes the $n$-weight of $\mu$.

If Conjecture 6.9 (ii) is true, this will prove that the formula $d_{\lambda^{\prime} \mu^{*}}=d_{\lambda \mu}$ is also satisfied by the decomposition numbers of Hecke algebras at roots of 1 , a reasonable statement which has already been conjectured $[33,12]$, and proved in case $n=p$ is a prime [38].

To prove this theorem we shall introduce, motivated by the previous discussion, two natural involutions of $\mathcal{F}$ and $M\left(\Lambda_{0}\right)$. The first one denoted by ' extends to $\mathcal{F}$ the conjugation of partitions, namely, we set

$$
\begin{aligned}
& \lambda^{\prime}=\text { the conjugate partition of } \lambda, \\
& (v+w)^{\prime}=v^{\prime}+w^{\prime}, \quad(v, w \in \mathcal{F}), \\
& (\varphi(q) v)^{\prime}=\varphi\left(q^{-1}\right) v^{\prime}, \quad(\varphi \in \mathbb{Q}(q)) .
\end{aligned}
$$

With this definition, the theorem we want to prove can be rewritten as

$$
G\left(\mu^{*}\right)=q^{|\mu|_{n}} G(\mu)^{\prime}
$$

The second involution comes from the automorphism ${ }^{\#}$ of $U_{q}\left(\widehat{\mathfrak{s l}}_{n}\right)$ specified by

$$
e_{i}^{\#}=e_{-i}, \quad f_{i}^{\#}=f_{-i}, \quad\left(q^{h_{t}}\right)^{\#}=q^{h_{-l}}, \quad\left(q^{D}\right)^{\#}=q^{D} .
$$

(It follows from the form of the generalized Cartan matrix $\left[\left\langle\alpha_{i}, h_{j}\right\rangle\right]$ which is symmetrical under $i \leftrightarrow-i \bmod n$ that these relations extend to an automorphism of $U_{q}\left(\widehat{\mathfrak{s l}}_{n}\right)$.) This automorphism induces an involution still denoted by ${ }^{\#}$ of $M\left(\Lambda_{0}\right)$ by setting

$$
\emptyset^{\#}=\emptyset, \quad(P v)^{\#}=P^{\#} v^{\#}, \quad\left(P \in U_{q}\left(\widehat{\mathfrak{s l}}_{n}\right), v \in M\left(\Lambda_{0}\right)\right)
$$

Note that for $n=2,{ }^{\#}=\mathrm{id}$, which has to be compared to the fact that in characteristic 2 the sign character coincides with the trivial character of the symmetric group. We shall first prove:

Lemma 7.3. For $\mu \in \mathcal{P}_{n}, G(\mu)^{\#}=G\left(\mu^{*}\right)$.

Proof of Lemma 7.3. We know that $G(\mu)$ is the unique vector of $M\left(\Lambda_{0}\right)_{\mathbb{Q}}$ satisfying

$$
\overline{G(\mu)}=G(\mu), \quad G(\mu) \equiv \tilde{f}_{r_{s}}^{k_{s}} \cdots \tilde{f}_{r_{1}}^{k_{1}} \emptyset \bmod q L\left(\Lambda_{0}\right),
$$

where $\emptyset \stackrel{r_{1}^{k_{1}}}{\longrightarrow} \ldots \stackrel{r_{s}^{k_{s}}}{\longrightarrow} \mu$ is a path from $\emptyset$ to $\mu$ in the crystal graph of $M\left(\Lambda_{0}\right)$.

Now, it is clear that the involutions ${ }^{\#}$ and - commute, so that $\overline{G(\mu)^{\#}}=(\overline{G(\mu)})^{\#}=$ $G(\mu)^{\#}$. Therefore, to prove Lemma 7.3 , it remains to show that

$$
G(\mu)^{\#} \equiv \tilde{f}_{-r_{s}}^{k_{s}} \cdots \tilde{f}_{-r_{1}}^{k_{1}} \emptyset \bmod q L\left(\Lambda_{0}\right) .
$$


This will result from

Lemma 7.4. For $v \in M\left(\Lambda_{0}\right),\left(\tilde{f}_{i} v\right)^{\#}=\tilde{f}_{-i}\left(v^{\#}\right)$.

Proof of Lemma 7.4. By linearity, we may assume that $v$ is a weight vector of weight $\Lambda$. The definition of $\tilde{f}_{i}$ (see Sect. 5) can be rephrased as follows. There exists a unique expansion

$$
v=\sum_{k} f_{i}^{(k)} u_{k}
$$

where $u_{k}$ has weight $\Lambda+k \alpha_{i}$ and $e_{i} u_{k}=0$, and one has

$$
\tilde{f_{i}} v=\sum_{k} f_{i}^{(k+1)} u_{k}
$$

Now, we have $v^{\#}=\sum_{k} f_{-i}^{(k)} u_{k}^{\#}$, where $e_{-i} u_{k}^{\#}=\left(e_{i} u_{k}\right)^{\#}=0$. Hence, $\tilde{f}_{-i} v^{\#}=$ $\sum_{k} f_{-i}^{(k+1)} u_{k}^{\#}=\left(\tilde{f_{i}} v\right)^{\#}$, as required.

Let us now conclude the proof of Lemma 7.3. It follows from Lemma 7.4 that

$$
L\left(\Lambda_{0}\right)=\sum_{i_{1},, i_{r}} A \tilde{f}_{i_{1}} \cdots \tilde{f}_{i_{r}} \emptyset
$$

is invariant under ${ }^{\#}$. Hence, $\left(q L\left(\Lambda_{0}\right)\right)^{\#}=q L\left(\Lambda_{0}\right)$, and

$$
G(\mu)^{\#} \equiv\left(\tilde{f}_{r_{s}}^{k_{s}} \cdots \tilde{f}_{r_{1}}^{k_{1}} \emptyset\right)^{\#} \bmod q L\left(\Lambda_{0}\right) \equiv \tilde{f}_{-r_{s}}^{k_{s}} \cdots \tilde{f}_{-r_{1}}^{k_{1}} \emptyset \bmod q L\left(\Lambda_{0}\right) .
$$

To prove the theorem, we have now to check that $G(\mu)^{\#}=q^{|\mu|_{n}} G(\mu)^{\prime}$. We first establish

Lemma 7.5. For $u \in \mathcal{F}, f_{i}^{\#} u=\left(q^{-1-h_{l}} f_{i} u^{\prime}\right)^{\prime}$.

Proof of Lemma 7.5: By linearity, it is enough to check it when $u=\lambda \in \mathcal{P}$. On the one hand, one has

$$
f_{i}^{\#} \lambda=f_{-i} \lambda=\sum_{v} q^{N_{-i}^{r}(\lambda, v)} v
$$

and on the other hand

$$
q^{-1-h_{i}} f_{i} \lambda^{\prime}=q^{-1-h_{i}} \sum_{v} q^{N_{i}^{r}\left(\lambda^{\prime}, v^{\prime}\right)} v^{\prime}=\sum_{v} q^{-1+N_{i}^{r}\left(\lambda^{\prime}, v^{\prime}\right)-N_{t}\left(v^{\prime}\right)} v^{\prime},
$$

so that all we have to check is

$$
N_{-i}^{r}(\lambda, v)=1-N_{i}^{r}\left(\lambda^{\prime}, v^{\prime}\right)+N_{i}\left(v^{\prime}\right) .
$$

Now, it is clear that $N_{-i}^{r}(\lambda, v)=N_{i}^{\ell}\left(\lambda^{\prime}, v^{\prime}\right)$, and taking into account the relation

$$
N_{i}\left(v^{\prime}\right)=N_{i}^{\ell}\left(\lambda^{\prime}, v^{\prime}\right)+N_{i}^{r}\left(\lambda^{\prime}, v^{\prime}\right)-1,
$$

the result follows. 
Using Lemma 7.5, we can now prove

Lemma 7.6. Let $v \in M\left(\Lambda_{0}\right)$ be a weight vector of weight $\Lambda$ satisfying $\bar{v}=v$. Then,

$$
v^{\#}=q^{-(\Lambda \mid \Lambda) / 2} v^{\prime}
$$

where $(\cdot \mid \cdot)$ denotes the usual symmetric bilinear form on the weight lattice of $\widehat{\mathfrak{s l}}_{n}($ see $[18])$.

Proof of Lemma 7.6. The hypothesis $\bar{v}=v$ means that $v$ is of the form

$$
v=\sum_{i_{1}, i_{k}} \beta_{i_{1}} i_{k}(q) f_{i_{1}} \cdots f_{i_{k}} \emptyset
$$

where $\beta_{i_{1}} i_{k}\left(q^{-1}\right)=\beta_{i_{1}} i_{k}(q)$ and $\Lambda=\Lambda_{0}-\alpha_{i_{1}}-\cdots-\alpha_{i_{k}}$. Thus, it is enough to prove the lemma in the case $v=f_{i_{1}} \cdots f_{i_{k}} \emptyset$. We proceed by induction on $k$ and assume that $u=f_{i_{2}} \cdots f_{i_{k}} \emptyset$ satisfies $u^{\#}=q^{-\left(\Lambda+\alpha_{i_{1}} \mid \Lambda+\alpha_{i_{1}}\right) / 2} u^{\prime}$. Then, by Lemma 7.5 ,

$$
v^{\#}=f_{i_{1}}^{\#} u^{\#}=q^{-\left(\Lambda+\alpha_{i_{1}} \mid \Lambda+\alpha_{t_{1}}\right) / 2} f_{i_{1}}^{\#} u^{\prime}=q^{-\left(\Lambda+\alpha_{i_{1}} \mid \Lambda+\alpha_{l_{1}}\right) / 2}\left(q^{-1-h_{l}} f_{i_{1}} u\right)^{\prime}
$$

So, $v^{\#}=q^{-\left(\Lambda+\alpha_{i_{1}} \mid \Lambda+\alpha_{i_{1}}\right) / 2} q^{1+\left(\Lambda \mid \alpha_{l_{1}}\right)} v^{\prime}=q^{-(\Lambda \mid \Lambda) / 2} v^{\prime}$ since $\left(\alpha_{i_{1}} \mid \alpha_{i_{1}}\right)=\left\langle\alpha_{i_{1}}, h_{i_{1}}\right\rangle=2$.

We can now finish the proof of the theorem. Since $\overline{G(\mu)}=G(\mu)$, we can apply Lemma 7.6, getting

$$
G(\mu)^{\#}=q^{-(\mathrm{wt} \mu \mid \mathrm{wt} \mu) / 2} G(\mu)^{\prime}=q^{|\mu|_{n}} G(\mu)^{\prime} .
$$

The second equality comes from the fact that

$$
\text { wt } \mu=w_{\theta}\left(\Lambda_{0}\right)-s \delta,
$$

where $\theta$ is the $n$-core of $\mu$ and $s=|\mu|_{n}$ (see Sect. 5). Therefore, since

$$
\begin{gathered}
\left(w_{\theta}\left(\Lambda_{0}\right) \mid w_{\theta}\left(\Lambda_{0}\right)\right)=\left(\Lambda_{0} \mid \Lambda_{0}\right)=0, \quad(\delta \mid \delta)=0, \\
\left(w_{\theta}\left(\Lambda_{0}\right) \mid \delta\right)=\left(\Lambda_{0} \mid w_{\theta}^{-1}(\delta)\right)=\left(\Lambda_{0} \mid \delta\right)=1,
\end{gathered}
$$

we see that $-($ wt $\mu \mid$ wt $\mu) / 2=|\mu|_{n}$.

Corollary 7.7. $d_{\lambda \mu}(q)$ is a polynomial of degree $\leqq|\mu|_{n}$. This inequality is strict for $\lambda \neq \mu^{* \prime}$, and $d_{\mu^{* \prime} \mu}(q)=q^{|\mu|_{n}}$. In particular, if $|\mu|_{n}=1$, there are only two nonzero entries in the column of $\mathbf{D}_{m}(q)$ indexed by $\mu$, namely $d_{\mu \mu}(q)=1$ and $d_{\mu^{* \prime} \mu}(q)=q$.

Proof. $\quad d_{\lambda^{\prime} \mu^{*}}(q)=q^{|\mu|_{n}} d_{\lambda \mu}\left(q^{-1}\right)$ contains no negative power of $q$, so $\operatorname{deg} d_{\lambda \mu}(q) \leqq$ $|\mu|_{n}$. Moreover, $d_{\lambda^{\prime} \mu^{*}}(0)=0$ except for $\lambda^{\prime}=\mu^{*}$ which shows that the inequality is strict if $\lambda \neq \mu^{* \prime}$. Finally, $d_{\mu^{* \prime} \mu}(q)=q^{|\mu|_{n}} d_{\mu^{*} \mu^{*}}(q)=q^{|\mu|_{n}}$.

Note that specializing $q$ to 1 , the last statement is in agreement with the description of decomposition numbers for blocks of weight 1 given by James [14].

Finally, we remark that from an algorithmic point of view, the theorem allows to reduce the computation of $\{G(\mu)\}$ to the determination of approximately half of it. 


\section{The Upper Global Crystal Basis of $M\left(\Lambda_{0}\right)$}

There is a natural scalar product, the so-called Shapovalov form, on the highest weight module $M\left(\Lambda_{0}\right)$, characterized by

$$
(\emptyset, \emptyset)=1, \quad\left(q^{h} u, v\right)=\left(u, q^{h} v\right), \quad\left(f_{i} u, v\right)=\left(u, e_{i} v\right),
$$

for $u, v \in M\left(\Lambda_{0}\right)$. The following remark is very useful.

Proposition 8.1. The scalar product $(\cdot, \cdot)$ on $M\left(\Lambda_{0}\right)$ is the restriction of the scalar product on $\mathcal{F}$ given by

$$
(\lambda, \mu)=q^{-|\lambda|_{n}} \delta_{\lambda \mu} .
$$

Proof. We have to show that the scalar product defined by (12) satisfies (11). By linearity, we may assume that $u=\lambda$ and $v=\mu$ belong to $\mathcal{P}$. First we have

$$
\left(q^{h_{i}} \lambda, \mu\right)=q^{N_{i}(\lambda)}(\lambda, \mu)=q^{N_{i}(\lambda)} q^{-|\lambda|_{n}} \delta_{\lambda \mu}=\left(\lambda, q^{h_{i}} \mu\right) .
$$

Next, if $\mu$ is not obtained from $\lambda$ by adding a $i$-node, then $\left(f_{i} \lambda, \mu\right)=0=\left(\lambda, e_{i} \mu\right)$. Finally, suppose that $\mu / \lambda$ is reduced to one $i$-node. Then, $\left(f_{i} \lambda, \mu\right)=q^{-|\mu|_{n}+N_{i}^{r}(\lambda, \mu)}$, while $\left(\lambda, e_{i} \mu\right)=q^{-|\lambda|_{n}} q^{-N_{i}^{\ell}(\lambda, \mu)}$. Now,

$$
\begin{aligned}
-|\lambda|_{n}=(\text { wt } \lambda \mid \text { wt } \lambda) / 2 & =\left(\text { wt } \mu+\alpha_{i} \mid \text { wt } \mu+\alpha_{i}\right) / 2=-|\mu|_{n}+1+\left(\text { wt } \mu \mid \alpha_{i}\right) \\
& =-|\mu|_{n}+1+N_{i}(\mu) .
\end{aligned}
$$

Therefore,

$$
-|\lambda|_{n}=-|\mu|_{n}+N_{i}^{r}(\lambda, \mu)+N_{i}^{\ell}(\lambda, \mu)
$$

as required.

It is interesting to note that equality (13) was already encountered by Robinson under the form

$$
|\mu|_{n}=|\lambda|_{n}+N_{i}(\lambda)-1
$$

in the modular representation theory of $\mathfrak{S}_{m}$ (see [39], p. 104).

The upper global crystal basis $\left\{G^{u p}(\mu), \mu \in \mathcal{P}_{n}\right\}$ of $M\left(\Lambda_{0}\right)$ is the adjoint basis of the global lower crystal basis $\{G(\mu)\}$ with respect to the scalar product $(\cdot, \cdot)$. That is, it is defined by $\left(G(\mu), G^{u p}(v)\right)=\delta_{\mu \nu}$. It may also be characterized in the following way [20]. The adjoint basis of $\{\lambda\}$ is clearly given by $\left\{q^{|\lambda|_{n}} \lambda\right\}$. Define the upper crystal lattice of $\mathcal{F}$ by

$$
L^{u p}=\bigoplus_{\lambda \in \mathcal{P}} A q^{|\lambda|_{n}} \lambda,
$$

and set $M\left(\Lambda_{0}\right)^{\mathbb{Q}}=\left\{v \in M\left(\Lambda_{0}\right) \mid\left(v, M\left(\Lambda_{0}\right)_{\mathbb{Q}}\right) \subset \mathbb{Q}\left[q, q^{-1}\right]\right\}$. Then, $G^{u p}(v)$ is the unique element of $M\left(\Lambda_{0}\right)^{\mathbb{Q}}$ satisfying

(i) $\overline{G^{u p}(\mu)}=G^{u p}(\mu)$,

(ii) $G^{u p}(\mu) \equiv q^{|\mu|_{n}} \mu \bmod q L^{u p}$. 
Example 8.2. Take $n=2$. The basis $\left\{G^{u p}(\mu)\right\}$ is given up to degree 4 by

$$
\begin{gathered}
G^{u p}(1)=(1), \\
G^{u p}(2)=\frac{1}{q+q^{-1}}\left((2)+q\left(1^{2}\right)\right), \\
G^{u p}(3)=\frac{1}{q+q^{-1}}\left((3)+q\left(1^{3}\right)\right), \\
G^{u p}(4)=\frac{1}{\left(q+q^{-1}\right)^{2}\left(q^{2}+q^{-2}\right)}\left(\left(q^{2}+1+q^{-2}\right)(4)+q^{3}(31)-\left(q^{2}+1\right)\left(2^{2}\right)\right. \\
G^{u p}(31)=\frac{\left.+q^{-1}\left(21^{2}\right)+\left(q^{4}+q^{2}+1\right)\left(1^{4}\right)\right),}{\left(q+q^{-1}\right)\left(q^{2}+q^{-2}\right)}\left(-(4)+q^{-1}(31)+\left(q^{2}+1\right)\left(2^{2}\right)\right. \\
\left.+q^{3}\left(21^{2}\right)-q^{2}\left(1^{4}\right)\right)
\end{gathered}
$$

In fact, it is more natural to regard $\left\{G^{u p}(\mu)\right\}$ as a basis of the dual space $\mathcal{F} / M\left(\Lambda_{0}\right)^{\perp} \cong M\left(\Lambda_{0}\right)$. Indeed, we have:

Proposition 8.3. For $\lambda \in \mathcal{P}$,

$$
q^{|\lambda|_{n}} \lambda \equiv \sum_{\mu \in \mathcal{P}_{n}} d_{\lambda \mu}(q) G^{u p}(\mu) \bmod M\left(\Lambda_{0}\right)^{\perp}
$$

Proof. The scalar product $(\cdot, \cdot)$ of $\mathcal{F}$ induces a pairing $(\cdot, \cdot)_{n}$ between $M\left(\Lambda_{0}\right)$ and $\mathcal{F} / M\left(\Lambda_{0}\right)^{\perp}$ given by

$$
\left(u, v \bmod M\left(\Lambda_{0}\right)^{\perp}\right)_{n}=(u, v), \quad\left(u \in M\left(\Lambda_{0}\right), v \in \mathcal{F}\right),
$$

which satisfies $\left(G(\mu), G^{u p}(v) \bmod M\left(\Lambda_{0}\right)^{\perp}\right)_{n}=\delta_{\mu \nu}$. We have $d_{\lambda \mu}(q)=\left(G(\mu), q^{|\lambda|_{n}} \lambda\right)$ On the other hand, write temporarily

$$
q^{|\lambda|_{n}} \lambda \bmod M\left(\Lambda_{0}\right)^{\perp}=\sum_{\mu \in \mathcal{P}_{n}} e_{\lambda \mu}(q)\left(G^{u p}(\mu) \bmod M\left(\Lambda_{0}\right)^{\perp}\right)
$$

Then,

$$
e_{\lambda \mu}(q)=\left(G(\mu), q^{|\lambda|_{n}} \lambda \bmod M\left(\Lambda_{0}\right)^{\perp}\right)_{n}=\left(G(\mu), q^{|\lambda|_{n}} \lambda\right)=d_{\lambda \mu}(q)
$$

as required. 
The proposition shows that we can rephrase Conjecture 6.9 as follows. The module $\mathcal{F} / M\left(\Lambda_{0}\right)^{\perp}$ at $q=1$ can be interpreted as the $\mathbb{Q}$-space $\mathcal{G}(\zeta) \otimes_{\mathbb{Z}} \mathbb{Q} \cong$ $\operatorname{Sym} / \mathcal{J}_{n} \otimes_{\mathbb{Z}} \mathbb{Q}$. This space is therefore endowed with a canonical basis, given by the classes $\phi_{\mu}$ of the irreducible $H_{m}(\zeta)$-modules $D(\mu)$. Our conjecture states that the canonical basis $\left\{\phi_{\mu}, \mu \in \mathcal{P}_{n}\right\}$ coincides with the specialization $q \rightarrow 1$ of the basis $\left\{G^{u p}(\mu) \bmod M\left(\Lambda_{0}\right)^{\perp}, \mu \in \mathcal{P}_{n}\right\}$ of $\mathcal{F} / M\left(\Lambda_{0}\right)^{\perp}$.

Finally, we remark that if the conjecture holds true, then the matrix

$$
\mathbf{C}\left(H_{m}(\zeta)\right)={ }^{\operatorname{tr}} \mathbf{D}\left(H_{m}(\zeta)\right) \mathbf{D}\left(H_{m}(\zeta)\right)=\left[c_{\mu \nu}\right]
$$

of the Cartan invariants of $H_{m}(\zeta)$ can be expressed as

$$
\mathbf{C}\left(H_{m}(\zeta)\right)=[(G(\mu), G(v))]_{q=1},
$$

that is, as the specialization at $q=1$ of the Gram matrix $\mathbf{C}_{m}(q)$ of the Shapovalov form in the lower crystal basis of $M\left(\Lambda_{0}\right)_{m}$.

\section{9. q-Decomposition Numbers and the Jantzen Filtration}

Assuming that Conjecture 6.9 holds, we see that we have obtained not only the decomposition matrix of $H_{m}(\zeta)$ but a $q$-analogue of it. A natural way of interpreting these $q$-decomposition numbers would be to find a filtration of the reduced Specht modules

$$
\overline{S(\lambda)}=S(\lambda)^{0} \geqq S(\lambda)^{1} \geqq S(\lambda)^{2} \geqq \cdots
$$

by $H_{m}(\zeta)$-submodules $S(\lambda)^{i}$ such that

$$
d_{\lambda \mu}(q)=\sum_{i \geqq 0}\left[S(\lambda)^{i} / S(\lambda)^{i+1}: D(\mu)\right] q^{i}
$$

We owe the following observation to Raphael Rouquier. Let (14) be the Jantzen filtration of $\overline{S(\lambda)}$ (see [17]). There exists a combinatorial formula for expressing the sum

$$
\sum_{i>0}\left[S(\lambda)^{i}\right] \in G_{0}\left(H_{m}(\zeta)\right)
$$

as a linear combination of $[\overline{S(\mu)}]$. If $(15)$ is true, then one must have

$$
\sum_{i>0}\left[S(\lambda)^{i}\right]=\sum_{\mu} d_{\lambda \mu}^{\prime}(1)[D(\mu)]
$$

(where $d_{\lambda \mu}^{\prime}(q)$ indicates the derivative with respect to $q$ of $d_{\lambda \mu}(q)$ ). A comparison of our tables with Jantzen's formula shows that (16) holds true. For example, when 
$\zeta=-1$ and $\lambda=\left(531^{2}\right)$, Jantzen's formula reads

$$
\begin{aligned}
\sum_{i>0}\left[S\left(531^{2}\right)^{i}\right] & =[\overline{S(532)}]+[\overline{S(631)}]+\left[\overline{S\left(5^{2}\right)}\right]+[\overline{S(10)}]-\left[\overline{S\left(71^{3}\right)}\right]+\left[\overline{S\left(621^{2}\right)}\right] \\
& =[D(532)]+3[D(631)]+4[D(64)]+[D(73)]+2[D(82)],
\end{aligned}
$$

while

$$
\begin{aligned}
q^{5}\left(531^{2}\right) \equiv & q G^{u p}(532)+q^{3} G^{u p}(631)+2 q^{2} G^{u p}(64)+q G^{u p}(73) \\
& +q^{2} G^{u p}(82) \bmod M\left(\Lambda_{0}\right)^{\perp}
\end{aligned}
$$

This gives a strong support for conjecturing that the $d_{\lambda \mu}(q)$ describe Jantzen's filtration of Specht modules.

\section{Tables}

This final section contains

the crystal graphs of the basic representation for $n=2, m \leqq 8, n=3, m \leqq 7$, the matrices $\mathbf{D}_{m}(q)$ for $n=2, m \leqq 13, n=3, m \leqq 10, n=4, m \leqq 10$, the matrices $\mathbf{C}_{m}(q)$ for $n=2, m \leqq 7, n=3, m \leqq 7$.

We note that tables giving the action of $e_{i}, f_{i}$ on $\left\{G^{u p}(\mu)\right\}$ for partitions with at most 3 parts in the case of $U_{q}\left(\widehat{\mathfrak{s l}}_{2}\right)$ have been computed in [6], Appendix 1.

10.1. The crystal graph of the basic representation of $U_{q}\left(\widehat{\mathfrak{s l}}_{2}\right)$

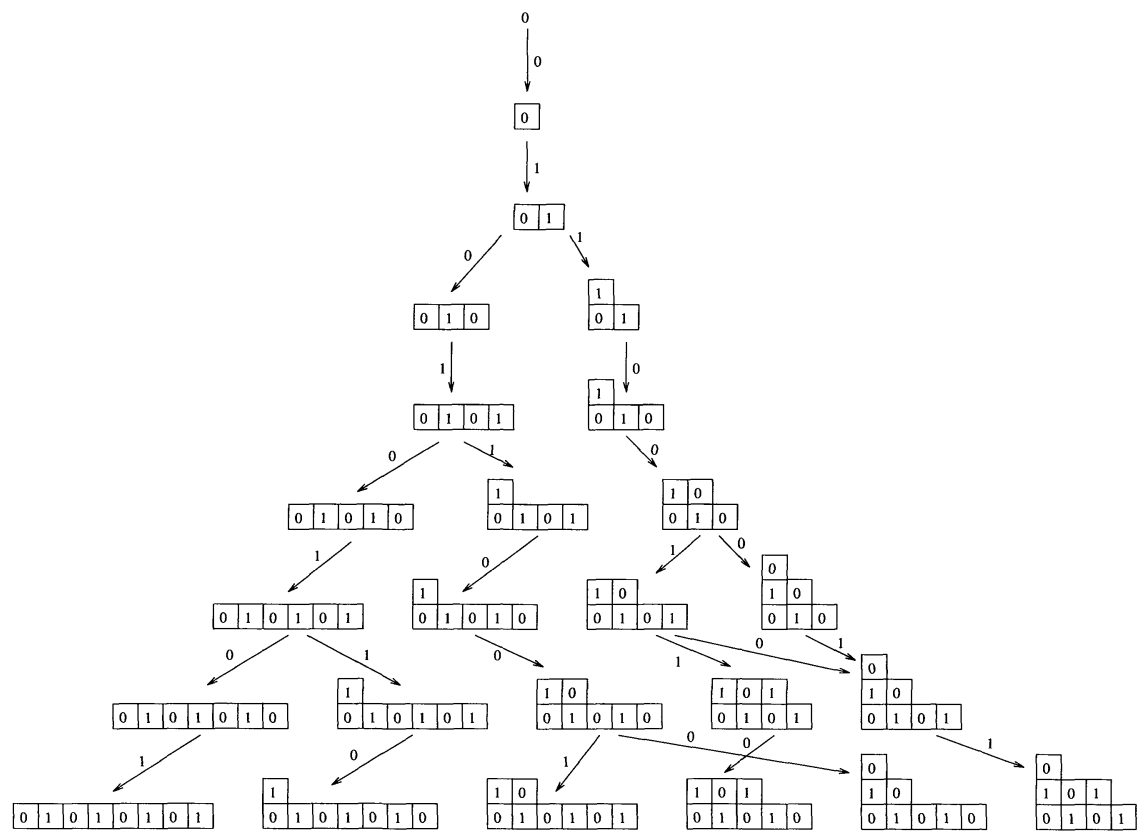


10.2. The crystal graph of the basic representation of $U_{q}\left(\widehat{\mathfrak{s}}_{3}\right)$

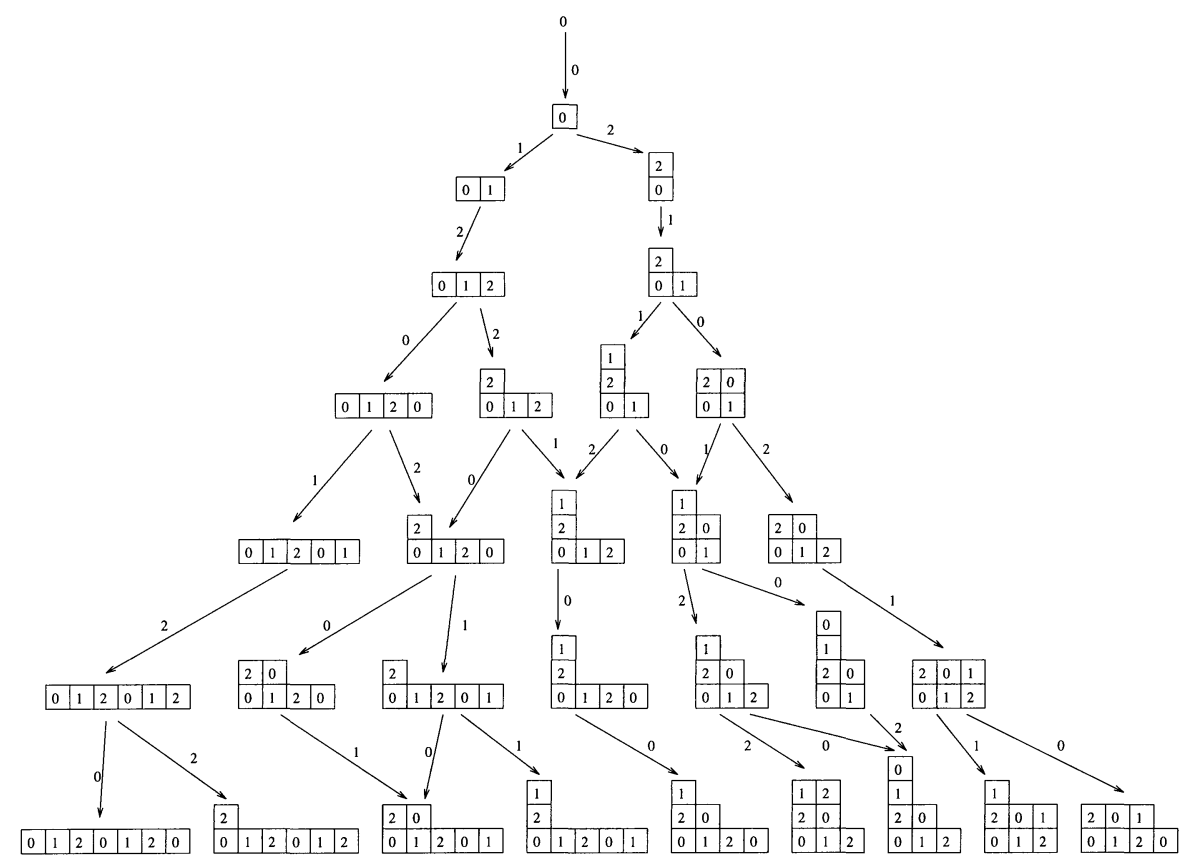

10.3. The global lower crystal basis of the basic representation of $U_{q}\left(\widehat{\mathfrak{s l}}_{2}\right)$

\begin{tabular}{|c|c|c|c|c|c|c|c|c|c|c|c|}
\hline & & & & & & & & & (5) & (41) & (32) \\
\hline & & & & & & & & (5) & 1 & 0 & 0 \\
\hline & & & & & & (4) $\quad 3$ & & (41) & 0 & 1 & 0 \\
\hline & & & (3) & (21) & (31) & $\begin{array}{l}1 \\
q\end{array}$ & & $\begin{array}{l}(32) \\
(311)\end{array}$ & 0 & 0 & 1 \\
\hline & & 3) & 1 & 0 & (22) & $\begin{array}{l}4 \\
0\end{array}$ & & (221) & $\begin{array}{l}q \\
0\end{array}$ & $\begin{array}{l}0 \\
0\end{array}$ & $\begin{array}{c}q \\
q^{2}\end{array}$ \\
\hline $\begin{array}{l}(2) \\
1\end{array}$ & & 21) & 0 & 1 & (211) & $q$ & & (2111) & 0 & $q$ & $\begin{array}{c}q^{2} \\
0\end{array}$ \\
\hline $\begin{array}{cc}(2) & 1 \\
\text { (11) } & q\end{array}$ & & 11) & $q$ & 0 & (1111) & $q^{2}$ & & (11111 & $q^{2}$ & 0 & 0 \\
\hline & & & & & & & (7) & (61) & $(52)$ & (43) & (421) \\
\hline & & & & & & (7) & 1 & 0 & 0 & 0 & 0 \\
\hline & & & & & & (61) & 0 & 1 & 0 & 0 & 0 \\
\hline & & & & & & (52) & 0 & 0 & 1 & 0 & 0 \\
\hline & (6) & (51) & (42) & (321) & & (511) & $q$ & 0 & $q$ & 0 & 0 \\
\hline (6) & 1 & 0 & 0 & 0 & & (43) & 0 & 0 & 0 & 1 & 0 \\
\hline (51) & $q$ & 1 & 0 & 0 & & (421) & 0 & 0 & $q^{2}$ & 0 & 1 \\
\hline (42) & 0 & $q$ & 1 & 0 & & (4111) & 0 & $q$ & 0 & $q$ & 0 \\
\hline (411) & $q$ & $q^{2}$ & $q$ & 0 & & $(331)$ & 0 & 0 & 0 & 0 & $q$ \\
\hline (33) & 0 & 0 & $q$ & 0 & & (322) & 0 & 0 & 0 & 0 & $q^{2}$ \\
\hline$(321)$ & 0 & 0 & 0 & 1 & & (3211) & 0 & 0 & $q$ & 0 & $q^{3}$ \\
\hline (3111) & $q^{2}$ & $q$ & $q^{2}$ & 0 & & (31111) & $q^{2}$ & 0 & $q^{2}$ & 0 & 0 \\
\hline (222) & 0 & 0 & $q^{2}$ & 0 & & $(2221)$ & 0 & 0 & 0 & $q^{2}$ & 0 \\
\hline (2211) & 0 & $q^{2}$ & $q^{3}$ & 0 & & (22111) & 0 & 0 & $q^{3}$ & 0 & 0 \\
\hline$(21111)$ & $q^{2}$ & $q^{3}$ & 0 & 0 & & (211111) & 0 & $q^{2}$ & 0 & 0 & 0 \\
\hline (111111) & $q^{3}$ & 0 & 0 & 0 & & (1111111) & $q^{3}$ & 0 & 0 & 0 & 0 \\
\hline
\end{tabular}




$\begin{array}{ccccccc} & (8) & (71) & (62) & (53) & (521) & (431) \\ (8) & 1 & 0 & 0 & 0 & 0 & 0 \\ (71) & q & 1 & 0 & 0 & 0 & 0 \\ (62) & 0 & q & 1 & 0 & 0 & 0 \\ (611) & q & q^{2} & q & 0 & 0 & 0 \\ (53) & 0 & 0 & q & 1 & 0 & 0 \\ (521) & 0 & 0 & 0 & 0 & 1 & 0 \\ (5111) & q^{2} & q & q^{2} & q & 0 & 0 \\ (44) & 0 & 0 & 0 & q & 0 & 0 \\ (431) & 0 & 0 & q & q^{2} & 0 & 1 \\ (422) & 0 & 0 & q^{2} & 0 & 0 & q \\ (4211) & 0 & q^{2} & q^{3}+q & q^{2} & 0 & q^{2} \\ (41111) & q^{2} & q^{3} & q^{2} & q^{3} & 0 & 0 \\ (332) & 0 & 0 & 0 & 0 & 0 & q^{2} \\ (3311) & 0 & 0 & q^{2} & 0 & 0 & q^{3} \\ (3221) & 0 & 0 & q^{3} & q^{2} & 0 & q^{4} \\ (32111) & 0 & 0 & 0 & 0 & q & 0 \\ (311111) & q^{3} & q^{2} & q^{3} & 0 & 0 & 0 \\ (2222) & 0 & 0 & 0 & q^{3} & 0 & 0 \\ (22211) & 0 & 0 & q^{3} & q^{4} & 0 & 0 \\ (221111) & 0 & q^{3} & q^{4} & 0 & 0 & 0 \\ (2111111) & q^{3} & q^{4} & 0 & 0 & 0 & 0 \\ (11111111) & q^{4} & 0 & 0 & 0 & 0 & 0\end{array}$

$\begin{array}{ccccccccc} & (9) & (81) & (72) & (63) & (621) & (54) & (531) & (432) \\ (9) & 1 & 0 & 0 & 0 & 0 & 0 & 0 & 0 \\ (81) & 0 & 1 & 0 & 0 & 0 & 0 & 0 & 0 \\ (72) & 0 & 0 & 1 & 0 & 0 & 0 & 0 & 0 \\ (711) & q & 0 & q & 0 & 0 & 0 & 0 & 0 \\ (63) & 0 & 0 & 0 & 1 & 0 & 0 & 0 & 0 \\ (621) & 0 & 0 & q^{2} & 0 & 1 & 0 & 0 & 0 \\ (6111) & 0 & q & 0 & q & 0 & 0 & 0 & 0 \\ (54) & 0 & 0 & 0 & 0 & 0 & 1 & 0 & 0 \\ (531) & 0 & 0 & 0 & 0 & q & q & 1 & 0 \\ (522) & 0 & 0 & 0 & 0 & q^{2} & 0 & q & 0 \\ (5211) & 0 & 0 & q & 0 & q^{3} & q & q^{2} & 0 \\ (51111) & q^{2} & 0 & q^{2} & 0 & 0 & q^{2} & 0 & 0 \\ (441) & 0 & 0 & 0 & 0 & 0 & q^{2} & q & 0 \\ (432) & 0 & 0 & 0 & 0 & 0 & 0 & 0 & 1 \\ (4311) & 0 & 0 & 0 & q & 0 & 0 & 0 & q \\ (4221) & 0 & 0 & 0 & q^{2} & 0 & 0 & 0 & q^{2} \\ (42111) & 0 & 0 & q^{3} & 0 & q & q^{3} & q^{2} & 0 \\ (411111) & 0 & q^{2} & 0 & q^{2} & 0 & 0 & 0 & 0 \\ (333) & 0 & 0 & 0 & 0 & 0 & 0 & q^{2} & 0 \\ (3321) & 0 & 0 & 0 & 0 & 0 & 0 & 0 & q^{3} \\ (33111) & 0 & 0 & 0 & 0 & q^{2} & 0 & q^{3} & 0 \\ (3222) & 0 & 0 & 0 & 0 & 0 & q^{2} & q^{3} & 0 \\ (32211) & 0 & 0 & 0 & 0 & q^{3} & q^{3} & q^{4} & 0 \\ (321111) & 0 & 0 & q^{2} & 0 & q^{4} & 0 & 0 & 0 \\ (3111111) & q^{3} & 0 & q^{3} & 0 & 0 & 0 & 0 & 0 \\ (22221) & 0 & 0 & 0 & 0 & 0 & q^{4} & 0 & 0 \\ (222111) & 0 & 0 & 0 & q^{3} & 0 & 0 & 0 & 0 \\ (2211111) & 0 & 0 & q^{4} & 0 & 0 & 0 & 0 & 0 \\ (21111111) & 0 & q^{3} & 0 & 0 & 0 & 0 & 0 & 0 \\ (11111111) & q^{4} & 0 & 0 & 0 & 0 & 0 & 0 & 0\end{array}$




\begin{tabular}{|c|c|c|c|c|c|c|c|c|c|c|}
\hline & $(10)$ & $(91)$ & $(82)$ & (73) & $(721)$ & $(64)$ & $(631)$ & $(541)$ & $(532)$ & $(4321)$ \\
\hline (10) & 1 & 0 & 0 & 0 & 0 & 0 & 0 & 0 & 0 & 0 \\
\hline (91) & $q$ & 1 & 0 & 0 & 0 & 0 & 0 & 0 & 0 & 0 \\
\hline$(82)$ & 0 & $q$ & 1 & 0 & 0 & 0 & 0 & 0 & 0 & 0 \\
\hline (811) & $q$ & $q^{2}$ & $q$ & 0 & 0 & 0 & 0 & 0 & 0 & 0 \\
\hline (73) & 0 & 0 & $q$ & 1 & 0 & 0 & 0 & 0 & 0 & 0 \\
\hline (721) & 0 & 0 & 0 & 0 & 1 & 0 & 0 & 0 & 0 & 0 \\
\hline$(7111)$ & $q^{2}$ & $q$ & $q^{2}$ & $q$ & 0 & 0 & 0 & 0 & 0 & 0 \\
\hline (64) & 0 & 0 & 0 & $q$ & 0 & 1 & 0 & 0 & 0 & 0 \\
\hline$(631)$ & 0 & 0 & $q$ & $q^{2}$ & 0 & $q$ & 1 & 0 & 0 & 0 \\
\hline$(622)$ & 0 & 0 & $q^{2}$ & 0 & 0 & 0 & $q$ & 0 & 0 & 0 \\
\hline$(6211)$ & 0 & $q^{2}$ & $q^{3}+q$ & $q^{2}$ & 0 & $q$ & $q^{2}$ & 0 & 0 & 0 \\
\hline (61111) & $q^{2}$ & $q^{3}$ & $q^{2}$ & $q^{3}$ & 0 & $q^{2}$ & 0 & 0 & 0 & 0 \\
\hline$(55)$ & 0 & 0 & 0 & 0 & 0 & $q$ & 0 & 0 & 0 & 0 \\
\hline (541) & 0 & 0 & 0 & 0 & 0 & 0 & 0 & 1 & 0 & 0 \\
\hline (532) & 0 & 0 & 0 & 0 & 0 & $q$ & $q^{2}$ & 0 & 1 & 0 \\
\hline (5311) & 0 & 0 & $q^{2}$ & $q$ & 0 & $2 q^{2}$ & $q^{3}$ & 0 & $q$ & 0 \\
\hline$(5221)$ & 0 & 0 & $q^{3}$ & $q^{2}$ & 0 & $q^{3}$ & $q^{4}$ & 0 & $q^{2}$ & 0 \\
\hline$(52111)$ & 0 & 0 & 0 & 0 & $q$ & 0 & 0 & $q$ & 0 & 0 \\
\hline (511111) & $q^{3}$ & $q^{2}$ & $q^{3}$ & $q^{2}$ & 0 & $q^{3}$ & 0 & 0 & 0 & 0 \\
\hline$(442)$ & 0 & 0 & 0 & 0 & 0 & $q^{2}$ & 0 & 0 & $q$ & 0 \\
\hline (4411) & 0 & 0 & 0 & $q^{2}$ & 0 & $q^{3}$ & 0 & 0 & $q^{2}$ & 0 \\
\hline$(433)$ & 0 & 0 & 0 & 0 & 0 & 0 & 0 & 0 & $q^{2}$ & 0 \\
\hline$(4321)$ & 0 & 0 & 0 & 0 & 0 & 0 & 0 & 0 & 0 & 1 \\
\hline (43111) & 0 & 0 & $q^{2}$ & $q^{3}$ & 0 & $q^{2}$ & $q$ & 0 & $q^{3}$ & 0 \\
\hline$(4222)$ & 0 & 0 & 0 & $q^{3}$ & 0 & $q^{2}$ & 0 & 0 & $q^{3}$ & 0 \\
\hline$(42211)$ & 0 & 0 & $q^{3}$ & $q^{4}$ & 0 & $2 q^{3}$ & $q^{2}$ & 0 & $q^{4}$ & 0 \\
\hline$(421111)$ & 0 & $q^{3}$ & $q^{4}+q^{2}$ & $q^{3}$ & 0 & $q^{4}$ & $q^{3}$ & 0 & 0 & 0 \\
\hline (4111111) & $q^{3}$ & $q^{4}$ & $q^{3}$ & $q^{4}$ & 0 & 0 & 0 & 0 & 0 & 0 \\
\hline (3331) & 0 & 0 & 0 & 0 & 0 & 0 & 0 & 0 & $q^{3}$ & 0 \\
\hline (3322) & 0 & 0 & 0 & 0 & 0 & $q^{3}$ & 0 & 0 & $q^{4}$ & 0 \\
\hline (33211) & 0 & 0 & 0 & 0 & 0 & $q^{4}$ & $q^{3}$ & 0 & $q^{5}$ & 0 \\
\hline (331111) & 0 & 0 & $q^{3}$ & 0 & 0 & 0 & $q^{4}$ & 0 & 0 & 0 \\
\hline (32221) & 0 & 0 & 0 & 0 & 0 & 0 & 0 & $q^{2}$ & 0 & 0 \\
\hline (322111) & 0 & 0 & $q^{4}$ & $q^{3}$ & 0 & $q^{4}$ & $q^{5}$ & 0 & 0 & 0 \\
\hline (3211111) & 0 & 0 & 0 & 0 & $q^{2}$ & 0 & 0 & 0 & 0 & 0 \\
\hline (31111111) & $q^{4}$ & $q^{3}$ & $q^{4}$ & 0 & 0 & 0 & 0 & 0 & 0 & 0 \\
\hline$(22222)$ & 0 & 0 & 0 & 0 & 0 & $q^{4}$ & 0 & 0 & 0 & 0 \\
\hline$(222211)$ & 0 & 0 & 0 & $q^{4}$ & 0 & $q^{5}$ & 0 & 0 & 0 & 0 \\
\hline (2221111) & 0 & 0 & $q^{4}$ & $q^{5}$ & 0 & 0 & 0 & 0 & 0 & 0 \\
\hline (22111111) & 0 & $q^{4}$ & $q^{5}$ & 0 & 0 & 0 & 0 & 0 & 0 & 0 \\
\hline 211111111) & $q^{4}$ & $q^{5}$ & 0 & 0 & 0 & 0 & 0 & 0 & 0 & 0 \\
\hline 111111111) & $q^{5}$ & 0 & 0 & 0 & 0 & 0 & 0 & 0 & 0 & 0 \\
\hline
\end{tabular}




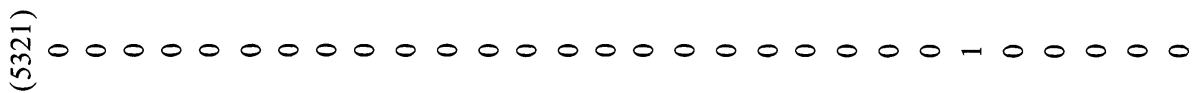

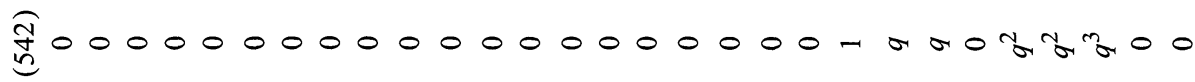
त్రై00000000000000- Wr 000000 H. 00000

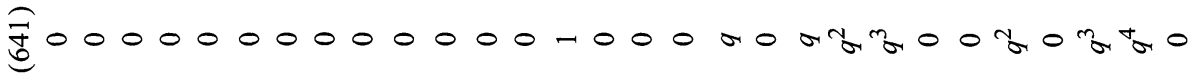

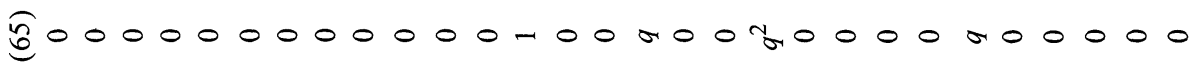
స్

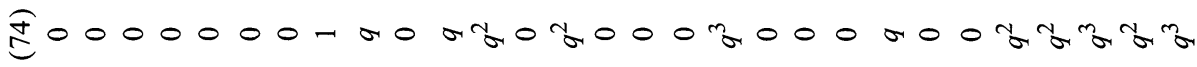

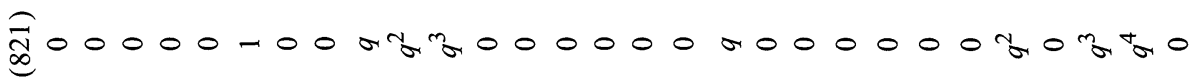

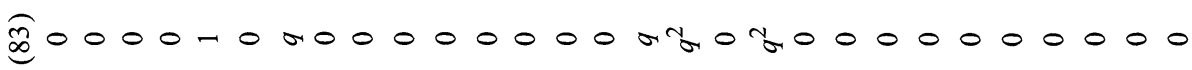
बूర0 -

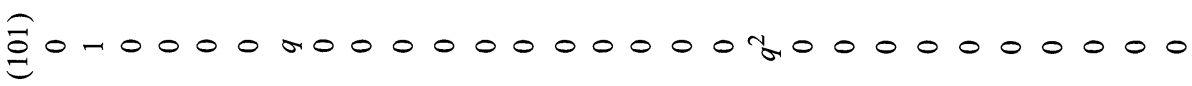

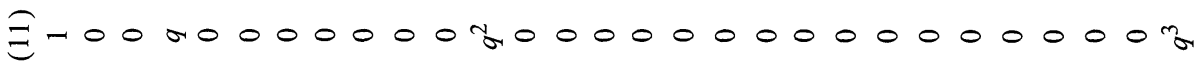

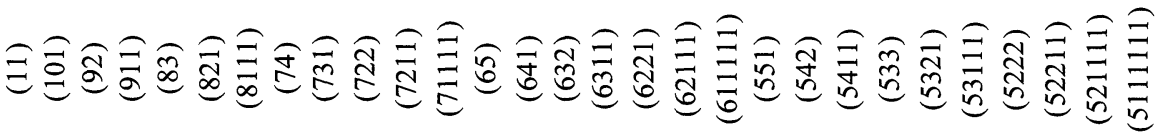


岕 胥

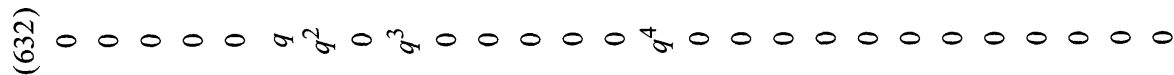

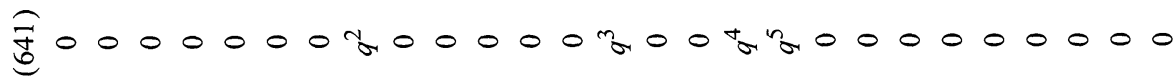

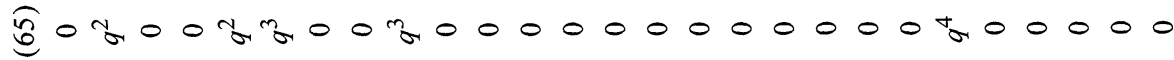

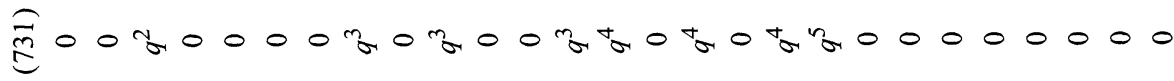

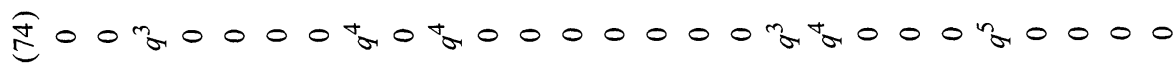

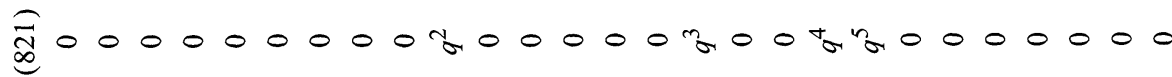

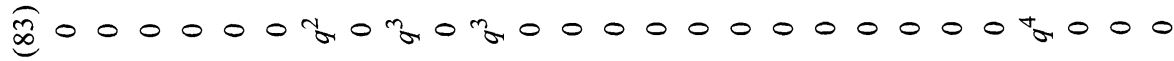
I $000000000 \pi_{0} 000000000 m_{4} 000,300$

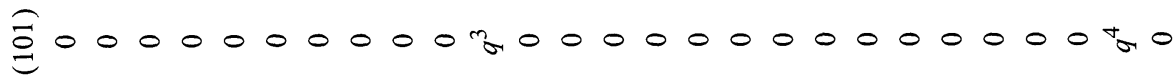
$\Xi 00000000000000000000 \operatorname{H}_{0} 00000$ 歹

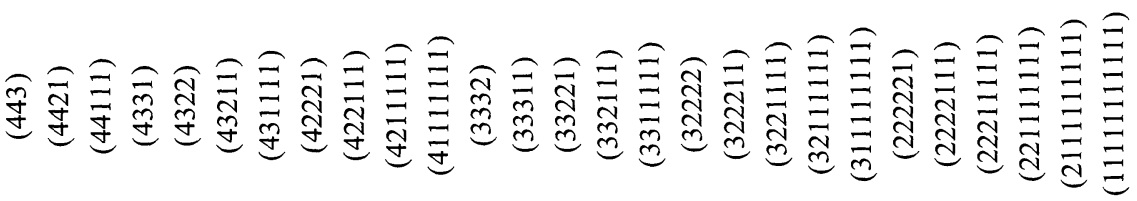


胥 00000000000000000000000000000 兼00000000000000000000000000000 $\underset{\text { तु }}{\widehat{d}} 000000000000000000000000-0000$ 胥000000000000000000000-

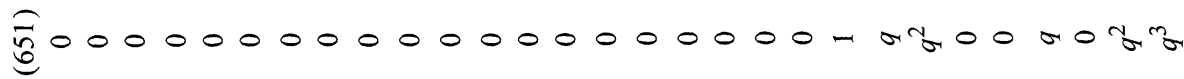
Iี $\stackrel{\vec{E}}{E} 000000000000-000000000000000$

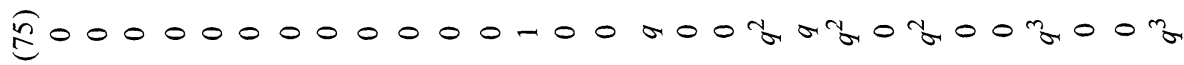

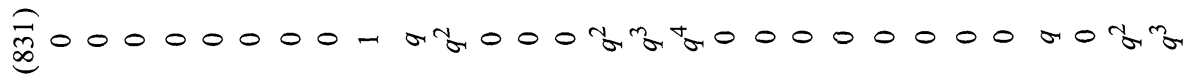

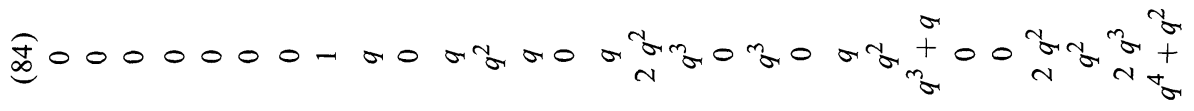
बूa $0000-00000000000000000000000$

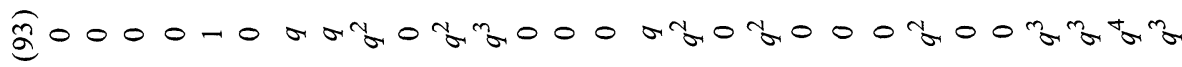

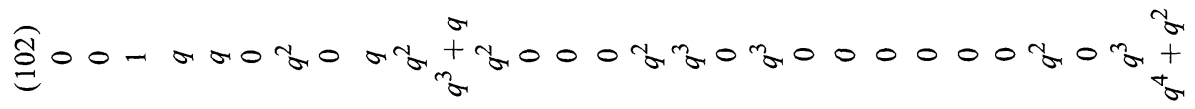
छ0I-

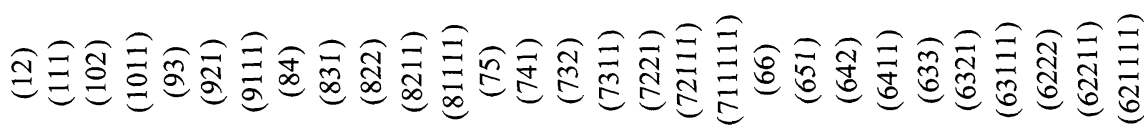




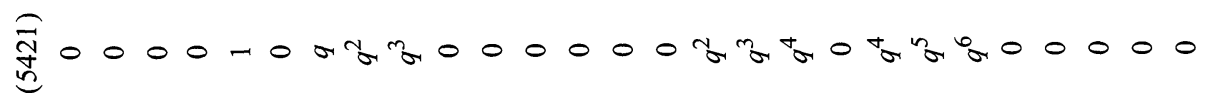
胥 $000-0,0000$ V

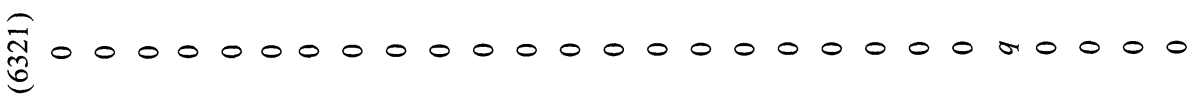

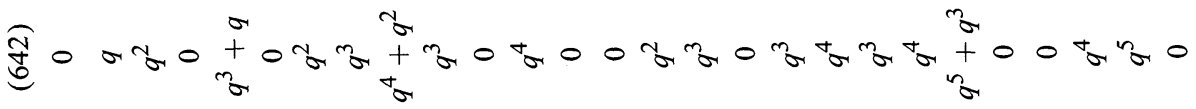

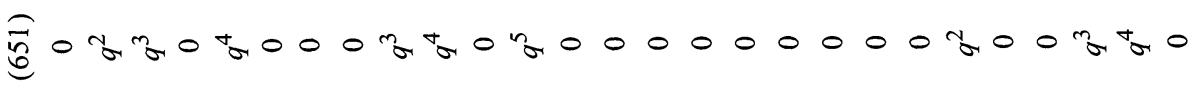

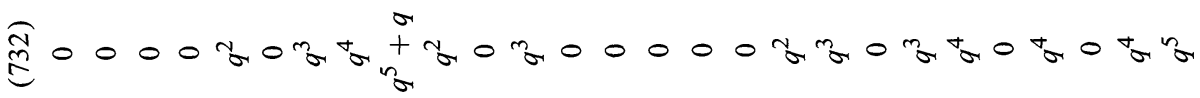
胥 $00000,0000 \gamma_{0} 0 \gamma_{0} 0000000000000$

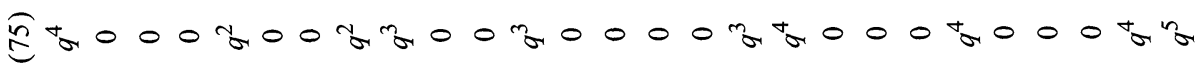

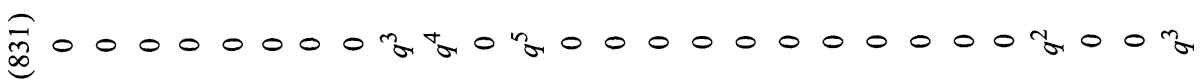

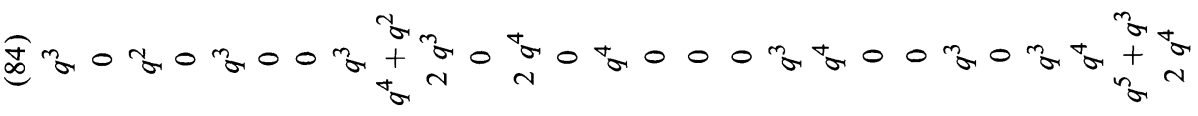
$\widehat{\widehat{\sigma}} 000000000000 \gamma 00000000000000$

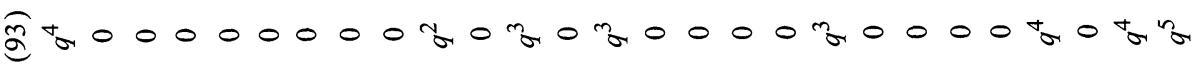
ฮิ $\Xi \sigma_{0} 000000000000 M_{0} 0000000000000$ İ $300000000000 H_{0} 0000000000000$

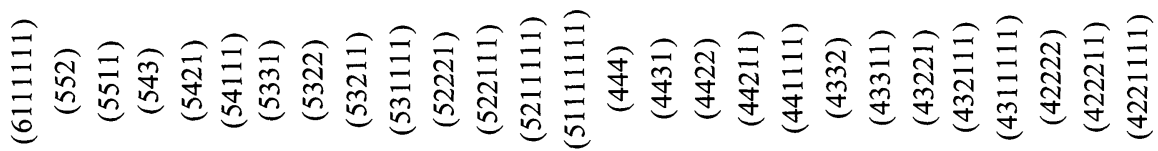




$$
\begin{aligned}
& 0000000000000000000000000000
\end{aligned}
$$

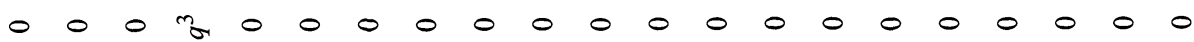

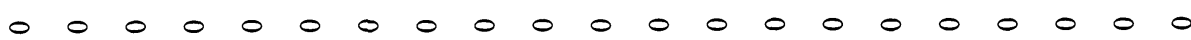

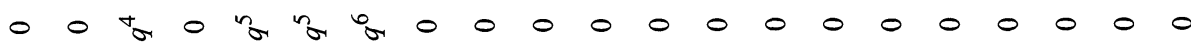

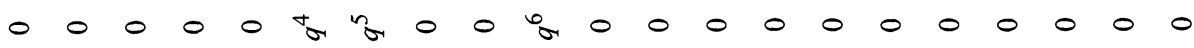

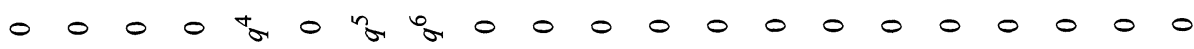

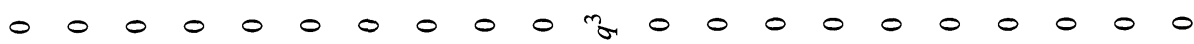

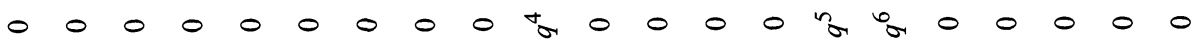

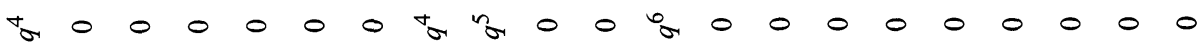

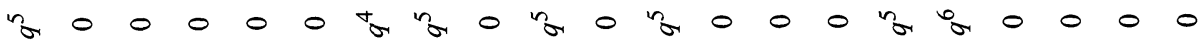

$$
\begin{aligned}
& 0000000000000 \text { m } 000000000000
\end{aligned}
$$

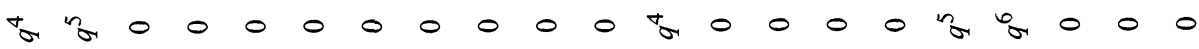

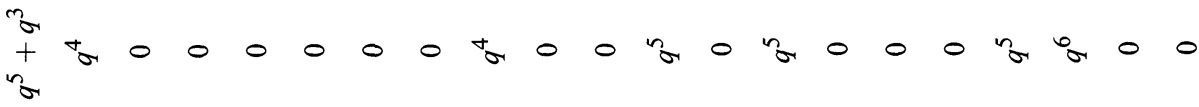

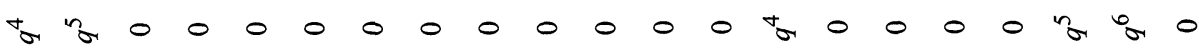

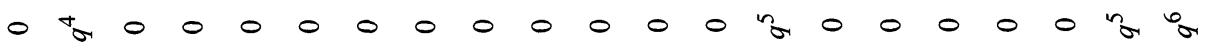

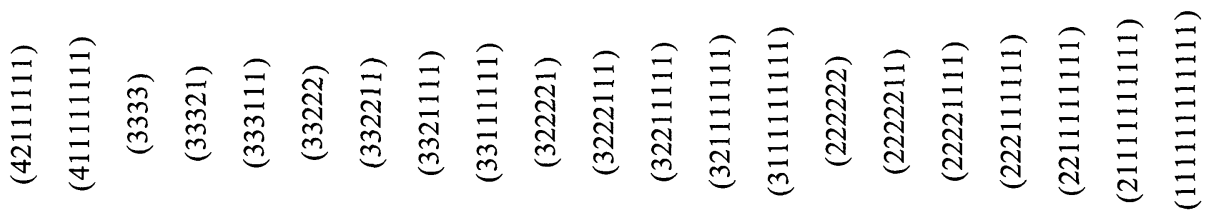


兽 000000000000000000000000000000000000 胥 $000000000000000000000000000000000-0$ fु $000000000000000000000000000000000-00$

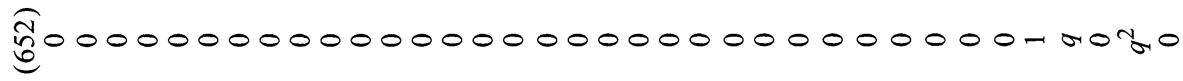
त् Iิ

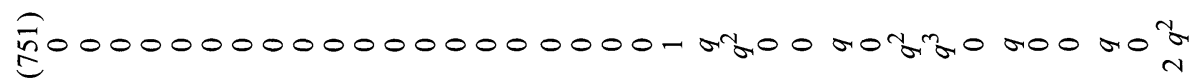

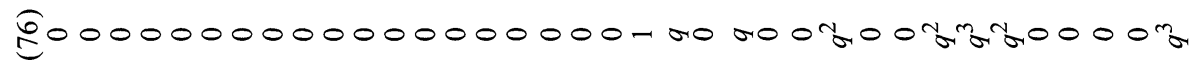

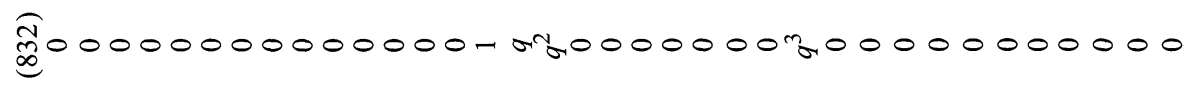

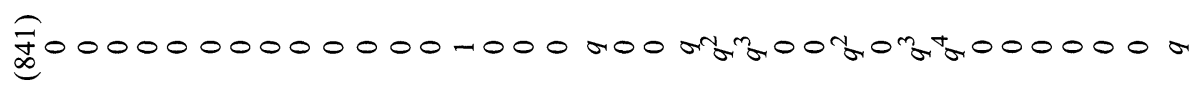

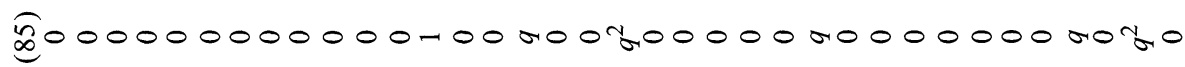

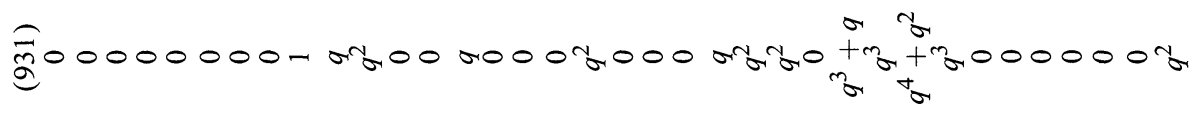

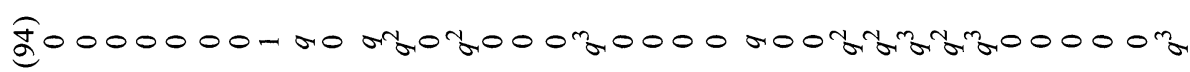

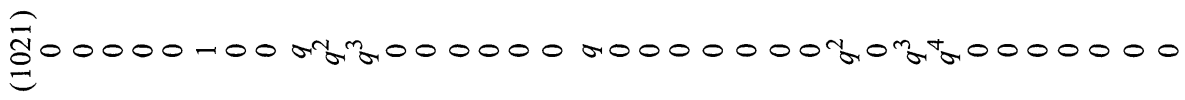

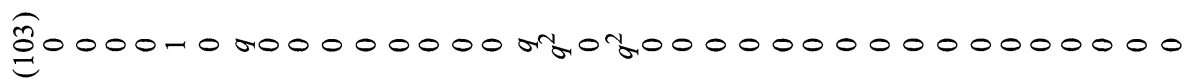
I00-

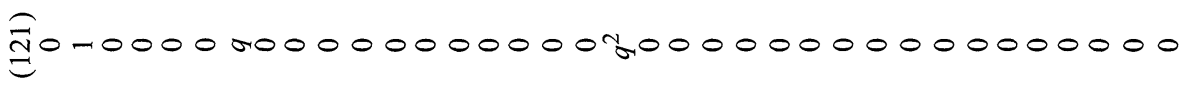
$\stackrel{\tilde{m}}{=}-0000000000\} 000000000000000003000000$

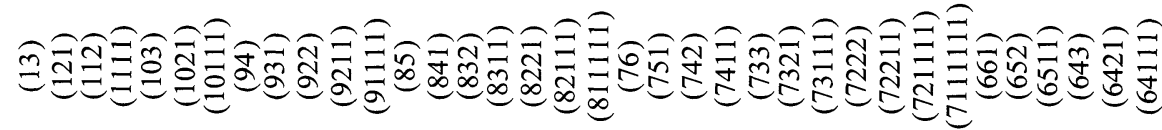




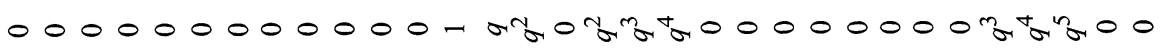

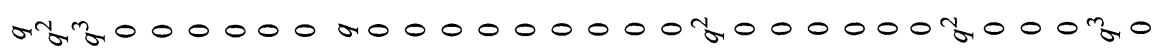

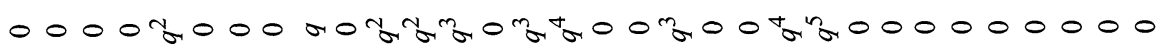
00 Rु, $0003000000000 \frac{\pi}{4} 00000000000$

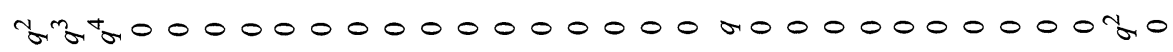

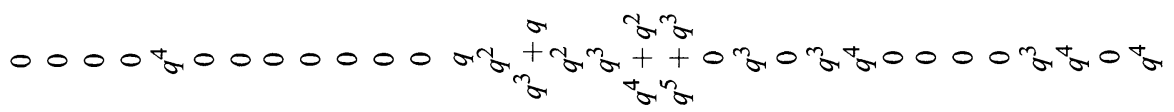

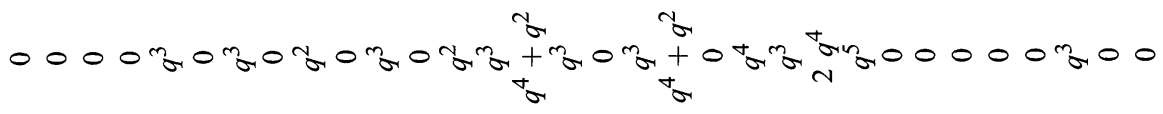

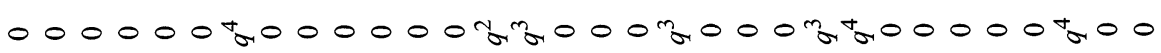
$00 R^{2} 0 \pi 0000000000000 \pi_{0} 00000000000$

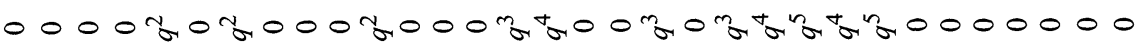

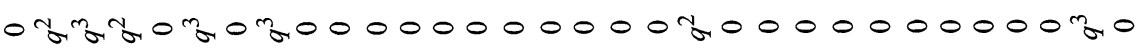

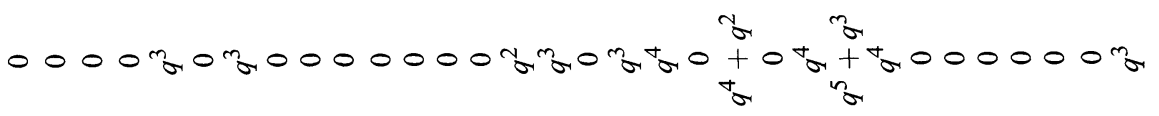

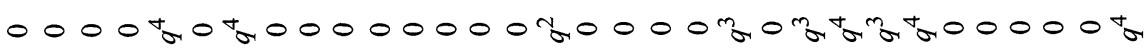
$000000 \pi 0000000000000300 \pi_{n} 0000000$ 000 \% $000000 H^{2} 00000000000000000 \pi_{4} 000000$ 00000003000000000000000000000000 $0000000000000000000000000 \operatorname{H}_{0} 000000$

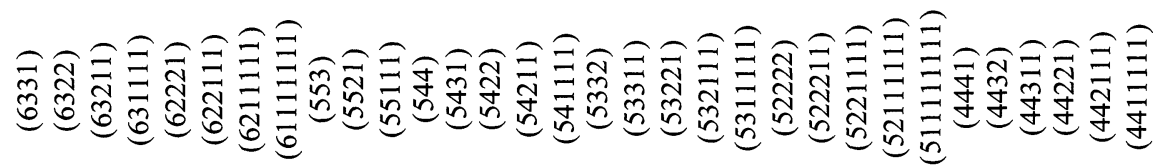


兽 000000000000000000000000000000000

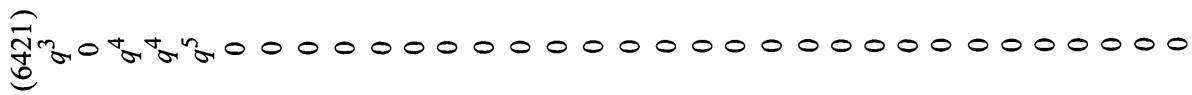

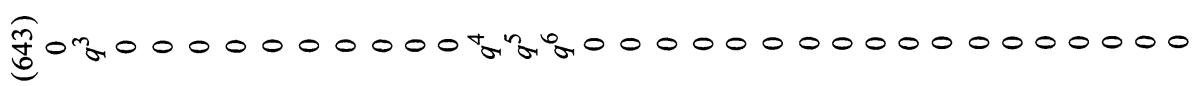
तु

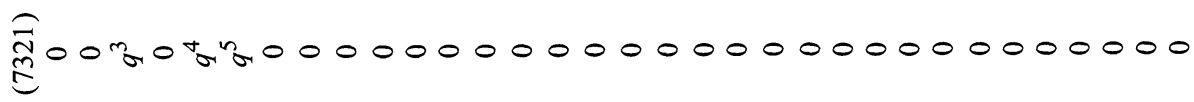
Iี है $200000000300000000000 \pi n 0000 \% 000000$

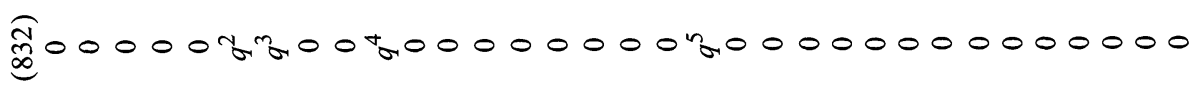

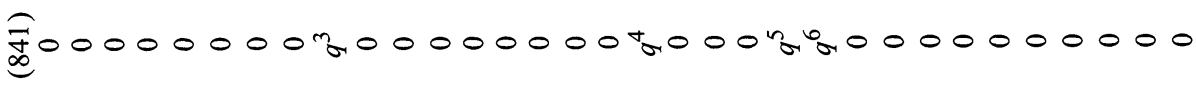

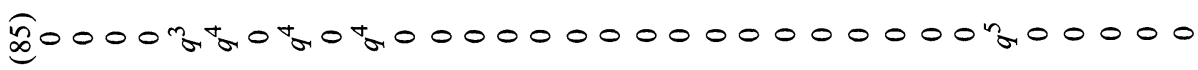

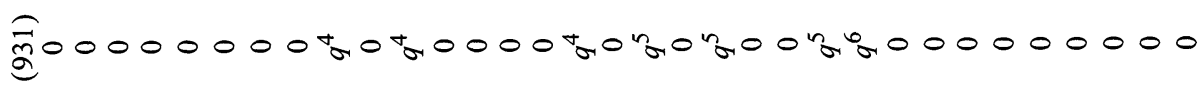

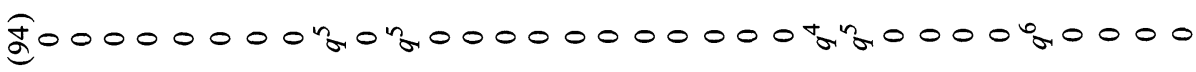

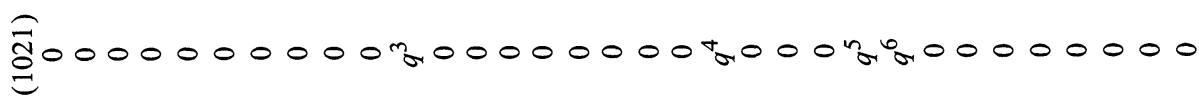

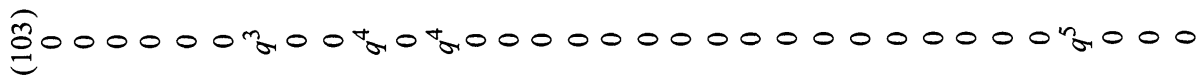
I000000000030000000000000\%n,

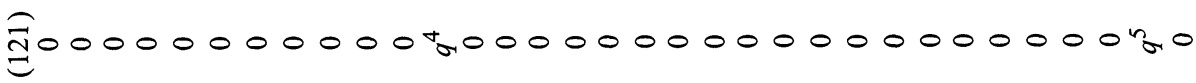
I্ 00000000000000000000000003000000 or

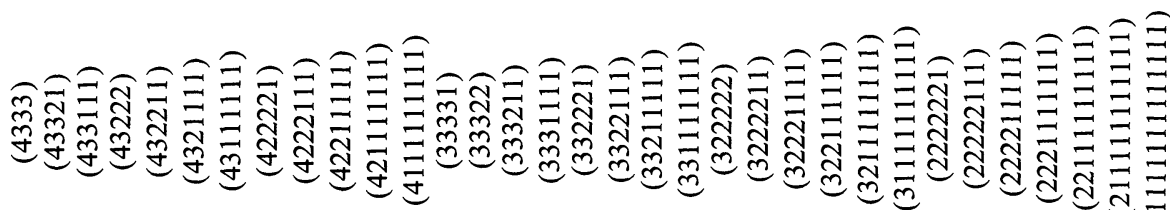


10.4. The global lower crystal basis of the basic representation of $U_{q}\left(\widehat{\mathfrak{s l}}_{3}\right)$

(2) $\begin{array}{cc}(2) & (11) \\ 1 & 0\end{array}$ (11) 001
(3) (21)

(3) 110
(4) (31) (22) (211)

$\begin{array}{lllll}(4) & 1 & 0 & 0 & 0 \\ \text { (31) } & 0 & 1 & 0 & 0\end{array}$

(22) $\quad q \quad 0 \quad 1 \quad 0$

$\begin{array}{lllll}(211) & 0 & 0 & 0 & 1\end{array}$

$\begin{array}{cccccc} & (5) & (41) & (32) & (311) & (221) \\ (5) & 1 & 0 & 0 & 0 & 0 \\ (41) & 0 & 1 & 0 & 0 & 0 \\ (32) & 0 & q & 1 & 0 & 0 \\ (311) & 0 & 0 & 0 & 1 & 0 \\ (221) & q & 0 & 0 & 0 & 1 \\ (2111) & 0 & 0 & 0 & 0 & q \\ (11111) & 0 & 0 & q & 0 & 0\end{array}$

$\begin{array}{cccccccc} & (6) & (51) & (42) & (411) & (33) & (321) & (2211) \\ (6) & 1 & 0 & 0 & 0 & 0 & 0 & 0 \\ (51) & q & 1 & 0 & 0 & 0 & 0 & 0 \\ (42) & 0 & 0 & 1 & 0 & 0 & 0 & 0 \\ (411) & 0 & q & 0 & 1 & 0 & 0 & 0 \\ (33) & 0 & q & 0 & 0 & 1 & 0 & 0 \\ (321) & q & q^{2} & 0 & q & q & 1 & 0 \\ (3111) & 0 & 0 & 0 & q^{2} & 0 & q & 0 \\ (222) & q^{2} & 0 & 0 & 0 & 0 & q & 0 \\ (2211) & 0 & 0 & 0 & 0 & 0 & 0 & 1 \\ (21111) & 0 & 0 & 0 & 0 & q & q^{2} & 0 \\ (111111) & 0 & 0 & 0 & 0 & q^{2} & 0 & 0\end{array}$

$\begin{array}{cccccccccc} & (7) & (61) & (52) & (511) & (43) & (421) & (331) & (322) & (3211) \\ (7) & 1 & 0 & 0 & 0 & 0 & 0 & 0 & 0 & 0 \\ (61) & 0 & 1 & 0 & 0 & 0 & 0 & 0 & 0 & 0 \\ (52) & q & 0 & 1 & 0 & 0 & 0 & 0 & 0 & 0 \\ (511) & 0 & 0 & 0 & 1 & 0 & 0 & 0 & 0 & 0 \\ (43) & 0 & 0 & q & 0 & 1 & 0 & 0 & 0 & 0 \\ (421) & q & 0 & q^{2} & 0 & q & 1 & 0 & 0 & 0 \\ (4111) & 0 & 0 & 0 & 0 & 0 & q & 0 & 0 & 0 \\ (331) & 0 & 0 & 0 & q & 0 & 0 & 1 & 0 & 0 \\ (322) & 0 & q & 0 & 0 & 0 & 0 & 0 & 1 & 0 \\ (3211) & q & 0 & 0 & 0 & q & q^{2} & 0 & 0 & 1 \\ (31111) & 0 & 0 & 0 & 0 & 0 & 0 & 0 & q & 0 \\ (2221) & q^{2} & 0 & 0 & 0 & 0 & 0 & 0 & 0 & q \\ (22111) & 0 & 0 & 0 & 0 & q & 0 & 0 & 0 & q^{2} \\ (211111) & 0 & 0 & 0 & 0 & 0 & 0 & q & 0 & 0 \\ (1111111) & 0 & 0 & 0 & 0 & q^{2} & 0 & 0 & 0 & 0\end{array}$




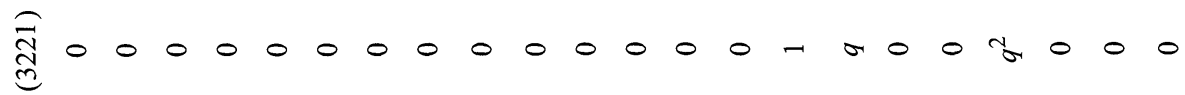

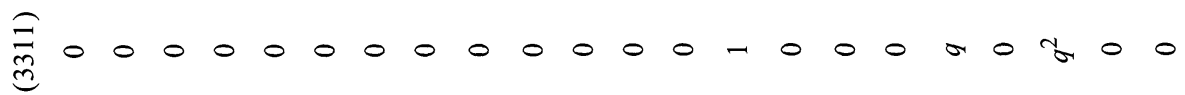

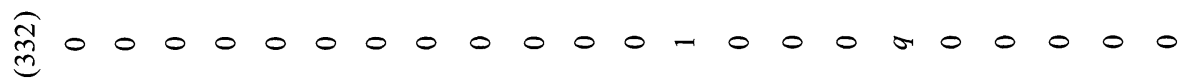

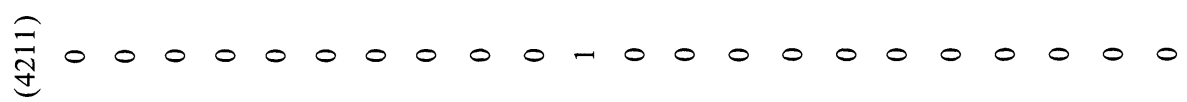

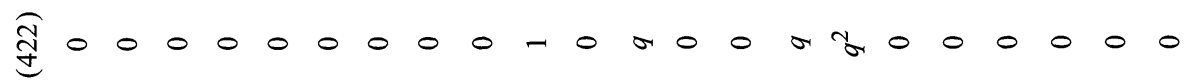
竎 $00000000-0000$ or 00000 or

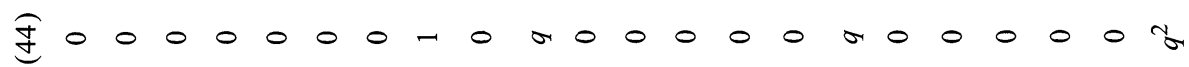

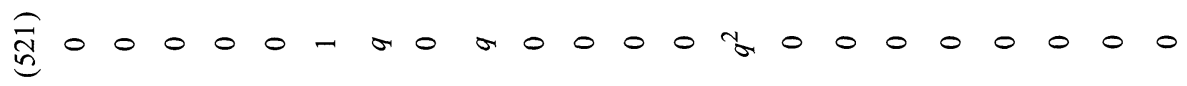

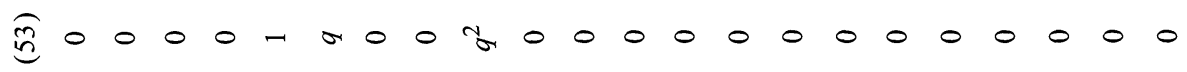

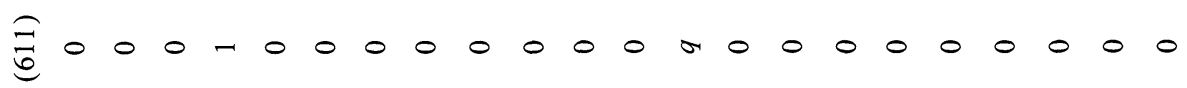

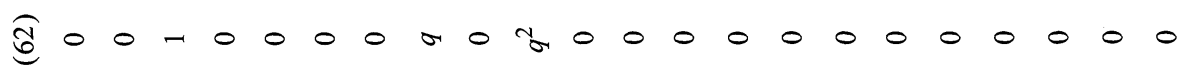
E 0 - 000000 a 0000 V 00000000

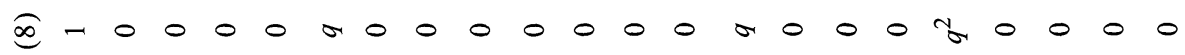

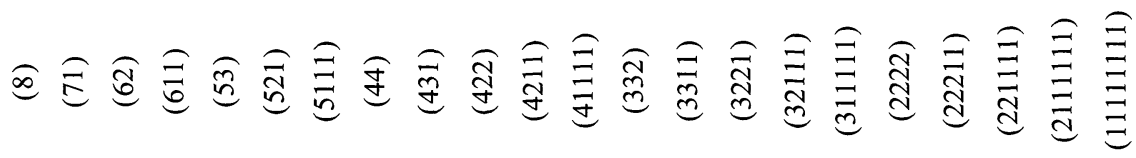


$\underset{\text { I }}{\stackrel{\text { I }}{J}} 0000000000000000000000-0000000$ $\underset{\widetilde{N}}{\tilde{N}} 0000000000000000000$ h

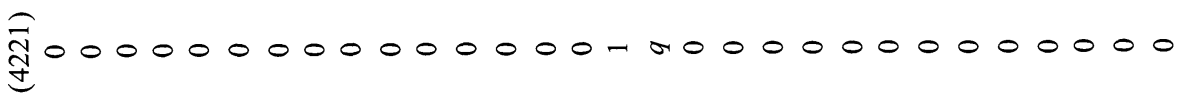
$\underset{\text { I }}{\text { I }} 00000000000000-000000000000000$

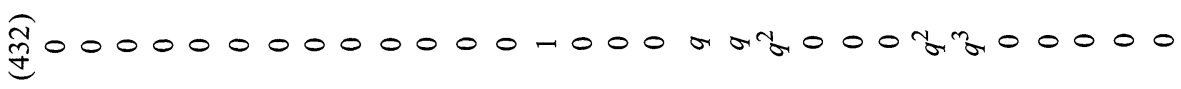

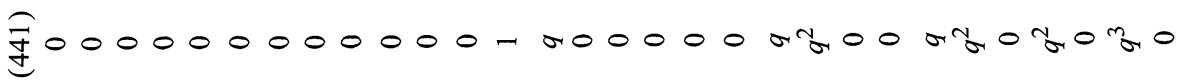

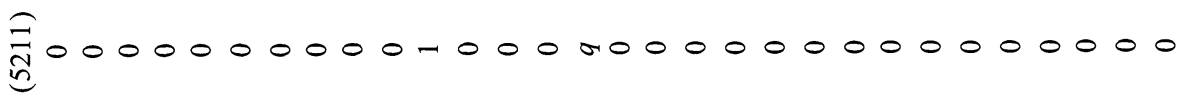

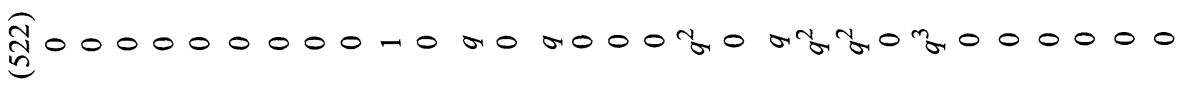
党 $00000000-000000000000000000000$

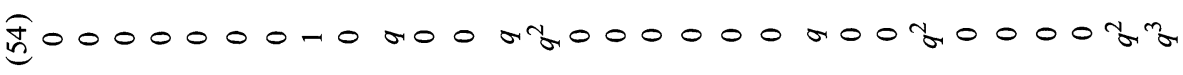

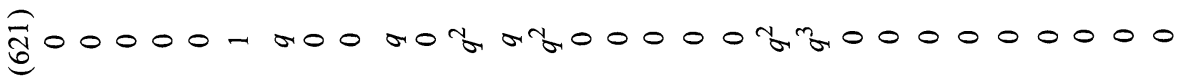

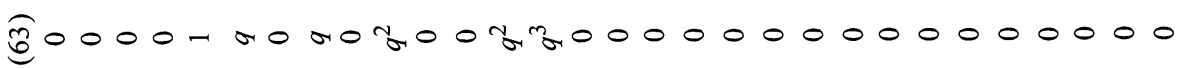
E000-0 aroo Iิ

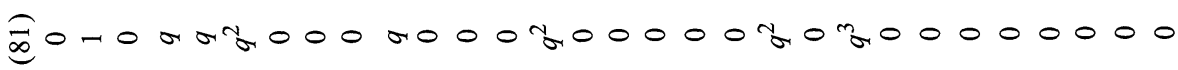
ब्l-

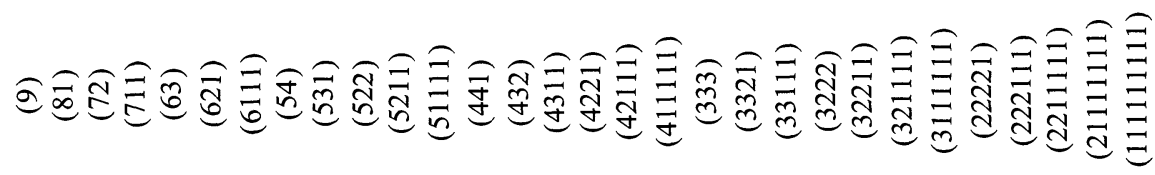


$\begin{array}{llllllllll}(10) & (91) & (82) & (811) & (73) & (721) & \text { (64) } & (631) & (622) & (6211)\end{array}$

$\begin{array}{llllllllllll}(10) & 1 & 0 & 0 & 0 & 0 & 0 & 0 & 0 & 0 & 0 & 0\end{array}$

(91) $\quad 0 \quad 1 \quad 00 \begin{array}{lllllllll} & 0 & 0 & 0 & 0 & 0 & 0 & 0 & 0\end{array}$

$\begin{array}{llllllllllll}\text { (82) } \quad q & 0 & 1 & 0 & 0 & 0 & 0 & 0 & 0 & 0 & 0\end{array}$

$\begin{array}{llllllllllll}(811) & 0 & 0 & 0 & 1 & 0 & 0 & 0 & 0 & 0 & 0 & 0\end{array}$

(73) $\quad 0 \quad 0 \quad \begin{array}{llllllllll} & 0 & 0 & 1 & 0 & 0 & 0 & 0 & 0 & 0\end{array}$

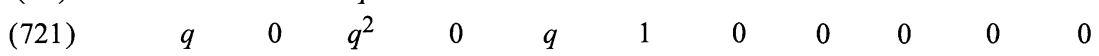

$\begin{array}{llllllllllll}(7111) & 0 & 0 & 0 & 0 & 0 & q & 0 & 0 & 0 & 0 & 0\end{array}$

$\begin{array}{llllllllllll}\text { (64) } & 0 & 0 & 0 & 0 & 0 & 0 & 1 & 0 & 0 & 0 & 0\end{array}$

$\begin{array}{llllllllllll}(631) & 0 & 0 & 0 & q & 0 & 0 & 0 & 1 & 0 & 0 & 0\end{array}$

$\begin{array}{llllllllllll}(622) & 0 & q & 0 & 0 & 0 & 0 & q & 0 & 1 & 0 & 0\end{array}$

$\begin{array}{llllllllllll}\text { (6211) } & q & 0 & 0 & 0 & q & q^{2} & 0 & 0 & 0 & 1 & 0\end{array}$

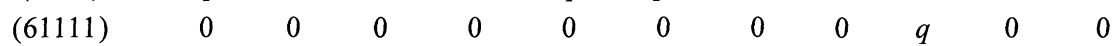

$\begin{array}{llllllllllll}(55) & 0 & 0 & 0 & 0 & q & 0 & 0 & 0 & 0 & 0 & 1\end{array}$

$\begin{array}{llllllllllll}(541) & 0 & 0 & 0 & 0 & 0 & 0 & 0 & q & 0 & 0 & 0\end{array}$

$\begin{array}{llllllllllll}(532) & 0 & 0 & 0 & q & 0 & 0 & 0 & q^{2} & 0 & 0 & 0\end{array}$

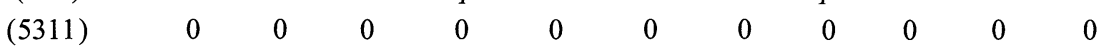

$\begin{array}{llllllllllll}(5221) & q^{2} & 0 & q & 0 & q^{2} & 0 & 0 & 0 & 0 & q & q\end{array}$

$\begin{array}{llllllllllll}(52111) & 0 & 0 & 0 & 0 & 0 & 0 & 0 & 0 & 0 & q^{2} & 0\end{array}$

$\begin{array}{llllllllllll}(511111) & 0 & 0 & 0 & 0 & 0 & 0 & 0 & 0 & 0 & 0 & 0\end{array}$

(442) $\quad 0 \quad 0 \quad 00 \begin{array}{lllllllll} & 0 & 0 & 0 & q^{2} & 0 & q & 0 & 0\end{array}$

$\begin{array}{llllllllllll}\text { (4411) } & 0 & 0 & 0 & 0 & q^{2} & 0 & 0 & 0 & 0 & q & q\end{array}$

$\begin{array}{llllllllllll}\text { (433) } & 0 & 0 & 0 & 0 & q^{2} & q & 0 & 0 & 0 & 0 & q\end{array}$

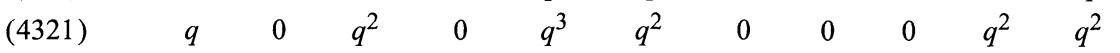

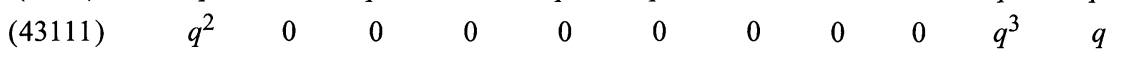

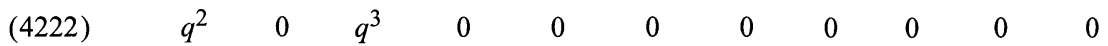

$\begin{array}{llllllllllll}(42211) & 0 & 0 & 0 & 0 & 0 & 0 & 0 & 0 & 0 & 0 & 0\end{array}$

$\begin{array}{llllllllllll}\text { (421111) } & 0 & 0 & 0 & 0 & 0 & 0 & 0 & 0 & 0 & 0 & q^{2}\end{array}$

$\begin{array}{llllllllllll}\text { (4111111) } & 0 & 0 & 0 & 0 & 0 & 0 & 0 & 0 & 0 & 0 & 0\end{array}$

(3331) $\quad q^{2} \quad 0 \quad 00 \begin{array}{lllllllll} & 0 & 0 & q^{3} & 0 & 0 & 0 & 0 & 0\end{array}$

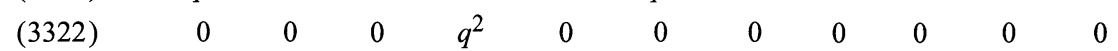

$\begin{array}{llllllllllll}(33211) & 0 & q & 0 & 0 & 0 & 0 & 0 & 0 & q^{2} & 0 & 0\end{array}$

$\begin{array}{llllllllllll}(331111) & 0 & 0 & 0 & 0 & 0 & 0 & 0 & 0 & 0 & 0 & 0\end{array}$

$\begin{array}{llllllllllll}(32221) & 0 & q^{2} & 0 & 0 & 0 & 0 & 0 & 0 & 0 & 0 & 0\end{array}$

$\begin{array}{llllllllllll}(322111) & 0 & 0 & 0 & 0 & 0 & 0 & 0 & 0 & 0 & 0 & 0\end{array}$

$\begin{array}{llllllllllll}(3211111) & 0 & 0 & 0 & 0 & 0 & 0 & 0 & 0 & 0 & 0 & q^{2}\end{array}$

$\begin{array}{llllllllllll}(31111111) & 0 & 0 & 0 & 0 & 0 & 0 & 0 & 0 & 0 & 0 & 0\end{array}$

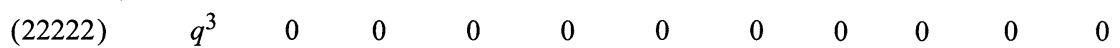

$\begin{array}{llllllllllll}\text { (222211) } & 0 & 0 & 0 & 0 & 0 & 0 & 0 & 0 & 0 & 0 & 0\end{array}$

$\begin{array}{llllllllllll}\text { (2221111) } & 0 & 0 & 0 & 0 & 0 & 0 & 0 & 0 & 0 & 0 & 0\end{array}$

$\begin{array}{llllllllllll}(22111111) & 0 & 0 & 0 & 0 & 0 & 0 & 0 & 0 & 0 & 0 & q^{2}\end{array}$

$\begin{array}{llllllllllll}(211111111) & 0 & 0 & 0 & 0 & 0 & 0 & 0 & 0 & 0 & 0 & 0\end{array}$

$\begin{array}{llllllllllll}(1111111111) & 0 & 0 & 0 & 0 & 0 & 0 & 0 & 0 & 0 & 0 & q^{3}\end{array}$ 
$\begin{array}{llllllllll}(541) & (532) & (5311) & (5221) & \text { (442) } & \text { (4411) } & \text { (433) } & \text { (4321) } & \text { (42211) (3322) } & \text { (33211) }\end{array}$

\begin{tabular}{|c|c|c|c|c|c|c|c|c|c|}
\hline 0 & 0 & 0 & 0 & 0 & 0 & 0 & 0 & 0 & 0 \\
\hline 0 & 0 & 0 & 0 & 0 & 0 & 0 & 0 & 0 & 0 \\
\hline 0 & 0 & 0 & 0 & 0 & 0 & 0 & 0 & 0 & 0 \\
\hline 0 & 0 & 0 & 0 & 0 & 0 & 0 & 0 & 0 & 0 \\
\hline 0 & 0 & 0 & 0 & 0 & 0 & 0 & 0 & 0 & 0 \\
\hline 0 & 0 & 0 & 0 & 0 & 0 & 0 & 0 & 0 & 0 \\
\hline 0 & 0 & 0 & 0 & 0 & 0 & 0 & 0 & 0 & 0 \\
\hline 0 & 0 & 0 & 0 & 0 & 0 & 0 & 0 & 0 & 0 \\
\hline 0 & 0 & 0 & 0 & 0 & 0 & 0 & 0 & 0 & 0 \\
\hline 0 & 0 & 0 & 0 & 0 & 0 & 0 & 0 & 0 & 0 \\
\hline 0 & 0 & 0 & 0 & 0 & 0 & 0 & 0 & 0 & 0 \\
\hline 0 & 0 & 0 & 0 & 0 & 0 & 0 & 0 & 0 & 0 \\
\hline 0 & 0 & 0 & 0 & 0 & 0 & 0 & 0 & 0 & 0 \\
\hline 1 & 0 & 0 & 0 & 0 & 0 & 0 & 0 & 0 & 0 \\
\hline$q$ & 1 & 0 & 0 & 0 & 0 & 0 & 0 & 0 & 0 \\
\hline 0 & 0 & 1 & 0 & 0 & 0 & 0 & 0 & 0 & 0 \\
\hline 0 & 0 & 0 & 1 & 0 & 0 & 0 & 0 & 0 & 0 \\
\hline 0 & 0 & 0 & $q$ & 0 & 0 & 0 & 0 & 0 & 0 \\
\hline 0 & $q$ & 0 & 0 & 0 & 0 & 0 & 0 & 0 & 0 \\
\hline 0 & 0 & 0 & 0 & 1 & 0 & 0 & 0 & 0 & 0 \\
\hline 0 & 0 & 0 & 0 & 0 & 1 & 0 & 0 & 0 & 0 \\
\hline 0 & 0 & 0 & 0 & 0 & 0 & 1 & 0 & 0 & 0 \\
\hline 0 & 0 & 0 & $q$ & 0 & $q$ & $q$ & 1 & 0 & 0 \\
\hline 0 & 0 & 0 & $q^{2}$ & 0 & $q^{2}$ & 0 & $q$ & 0 & 0 \\
\hline 0 & 0 & 0 & $q^{2}$ & 0 & 0 & 0 & $q$ & 0 & 0 \\
\hline 0 & 0 & 0 & 0 & 0 & 0 & 0 & 0 & 1 & 0 \\
\hline 0 & 0 & 0 & $q^{3}$ & 0 & 0 & $q$ & $q^{2}$ & 0 & 0 \\
\hline 0 & 0 & 0 & 0 & 0 & 0 & $q^{2}$ & 0 & 0 & 0 \\
\hline 0 & 0 & 0 & 0 & 0 & 0 & $q^{2}$ & $q$ & 0 & 0 \\
\hline 0 & $q$ & 0 & 0 & 0 & 0 & 0 & 0 & 0 & 1 \\
\hline 0 & 0 & 0 & 0 & $q$ & 0 & 0 & 0 & 0 & 0 \\
\hline$q$ & $q^{2}$ & 0 & 0 & 0 & 0 & 0 & 0 & 0 & $q$ \\
\hline 0 & 0 & 0 & 0 & 0 & 0 & 0 & 0 & 0 & 0 \\
\hline 0 & 0 & 0 & 0 & $q$ & 0 & 0 & 0 & 0 & 0 \\
\hline 0 & 0 & 0 & 0 & 0 & $q$ & $q^{3}$ & $q^{2}$ & 0 & 0 \\
\hline 0 & 0 & 0 & 0 & $q^{2}$ & 0 & 0 & 0 & 0 & 0 \\
\hline 0 & 0 & 0 & 0 & 0 & 0 & 0 & $q^{2}$ & 0 & 0 \\
\hline 0 & 0 & 0 & 0 & 0 & 0 & 0 & 0 & 0 & $q^{2}$ \\
\hline 0 & 0 & 0 & 0 & 0 & $q^{2}$ & 0 & $q^{3}$ & 0 & 0 \\
\hline 0 & 0 & 0 & 0 & 0 & $q^{3}$ & 0 & 0 & 0 & 0 \\
\hline$q^{2}$ & 0 & 0 & 0 & 0 & 0 & 0 & 0 & 0 & 0 \\
\hline 0 & 0 & 0 & 0 & 0 & 0 & 0 & 0 & 0 & 0 \\
\hline
\end{tabular}


10.5. The global lower crystal basis of the basic representation of $U_{q}\left(\widehat{\mathfrak{G l}}_{4}\right)$

\begin{tabular}{|c|c|c|c|c|c|c|c|c|c|c|c|}
\hline & & & & & & & \multicolumn{2}{|r|}{ (4) } & (31) & (22) & (211) \\
\hline & & & & & & & (4) & 1 & 0 & 0 & 0 \\
\hline & & & & (3) & (21) & (111) & (31) & $q$ & 1 & 0 & 0 \\
\hline & (2) & (11) & (3) & 1 & 0 & 0 & (22) & 0 & 0 & 1 & 0 \\
\hline (2) & 1 & 0 & (21) & 0 & 1 & 0 & (211) & 0 & $q$ & 0 & 1 \\
\hline (11) & 0 & 1 & (111) & 0 & 0 & 1 & (1111) & 0 & 0 & 0 & $q$ \\
\hline
\end{tabular}

$\begin{array}{ccccccc} & (5) & (41) & (32) & (311) & (221) & (2111) \\ (5) & 1 & 0 & 0 & 0 & 0 & 0 \\ (41) & 0 & 1 & 0 & 0 & 0 & 0 \\ (32) & q & 0 & 1 & 0 & 0 & 0 \\ (311) & 0 & 0 & 0 & 1 & 0 & 0 \\ (221) & 0 & 0 & q & 0 & 1 & 0 \\ (2111) & 0 & 0 & 0 & 0 & 0 & 1 \\ (11111) & 0 & 0 & 0 & 0 & q & 0\end{array}$

$\begin{array}{cccccccccc} & (6) & (51) & (42) & (411) & (33) & (321) & (3111) & (222) & (2211) \\ (6) & 1 & 0 & 0 & 0 & 0 & 0 & 0 & 0 & 0 \\ (51) & 0 & 1 & 0 & 0 & 0 & 0 & 0 & 0 & 0 \\ (42) & 0 & q & 1 & 0 & 0 & 0 & 0 & 0 & 0 \\ (411) & 0 & 0 & 0 & 1 & 0 & 0 & 0 & 0 & 0 \\ (33) & q & 0 & 0 & 0 & 1 & 0 & 0 & 0 & 0 \\ (321) & 0 & 0 & 0 & 0 & 0 & 1 & 0 & 0 & 0 \\ (3111) & 0 & 0 & 0 & 0 & 0 & 0 & 1 & 0 & 0 \\ (222) & 0 & 0 & q & 0 & 0 & 0 & 0 & 1 & 0 \\ (2211) & 0 & 0 & 0 & 0 & q & 0 & 0 & 0 & 1 \\ (21111) & 0 & 0 & 0 & 0 & 0 & 0 & 0 & 0 & q \\ (111111) & 0 & 0 & 0 & 0 & 0 & 0 & 0 & q & 0\end{array}$

$\begin{array}{ccccccccccccc} & (7) & (61) & (52) & (511) & (43) & (421) & (4111) & (331) & (322) & (3211) & (2221) & (22111) \\ (7) & 1 & 0 & 0 & 0 & 0 & 0 & 0 & 0 & 0 & 0 & 0 & 0 \\ (61) & 0 & 1 & 0 & 0 & 0 & 0 & 0 & 0 & 0 & 0 & 0 & 0 \\ (52) & 0 & 0 & 1 & 0 & 0 & 0 & 0 & 0 & 0 & 0 & 0 & 0 \\ (511) & 0 & 0 & 0 & 1 & 0 & 0 & 0 & 0 & 0 & 0 & 0 & 0 \\ (43) & 0 & q & 0 & 0 & 1 & 0 & 0 & 0 & 0 & 0 & 0 & 0 \\ (421) & 0 & 0 & 0 & q & 0 & 1 & 0 & 0 & 0 & 0 & 0 & 0 \\ (4111) & 0 & 0 & 0 & 0 & 0 & 0 & 1 & 0 & 0 & 0 & 0 & 0 \\ (331) & q & 0 & 0 & 0 & 0 & 0 & 0 & 1 & 0 & 0 & 0 & 0 \\ (322) & 0 & 0 & 0 & 0 & 0 & q & 0 & 0 & 1 & 0 & 0 & 0 \\ (3211) & 0 & 0 & 0 & 0 & 0 & 0 & 0 & q & 0 & 1 & 0 & 0 \\ (31111) & 0 & 0 & 0 & 0 & 0 & 0 & 0 & 0 & 0 & q & 0 & 0 \\ (2221) & 0 & 0 & 0 & 0 & q & 0 & 0 & 0 & 0 & 0 & 1 & 0 \\ (22111) & 0 & 0 & 0 & 0 & 0 & 0 & 0 & 0 & 0 & 0 & 0 & 1 \\ (211111) & 0 & 0 & 0 & 0 & 0 & 0 & 0 & 0 & 0 & 0 & q & 0 \\ (1111111) & 0 & 0 & 0 & 0 & 0 & 0 & 0 & 0 & q & 0 & 0 & 0\end{array}$




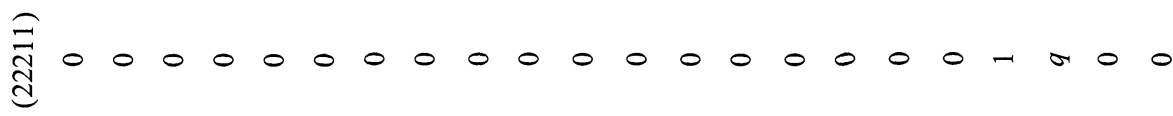

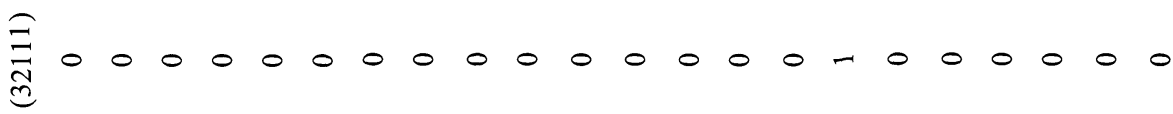

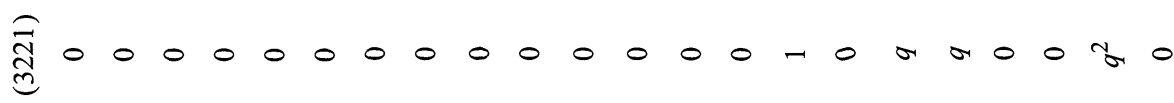

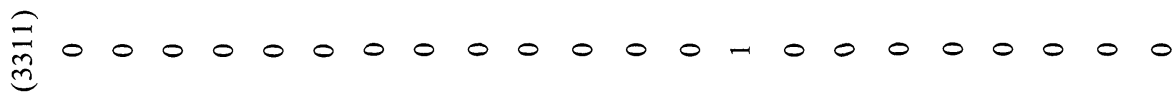

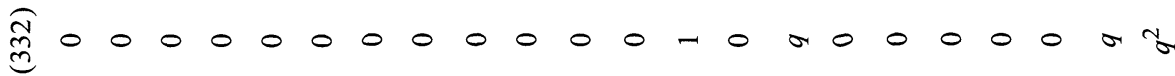

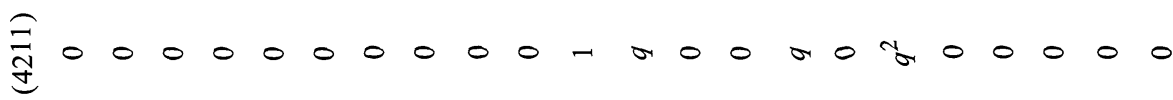

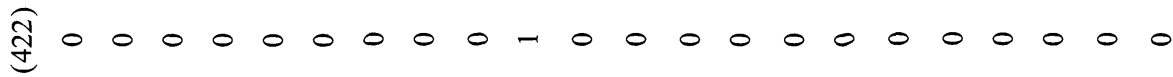

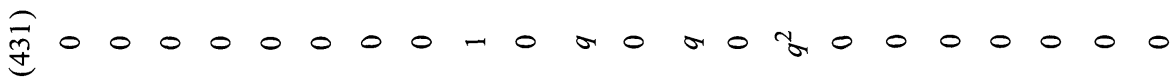

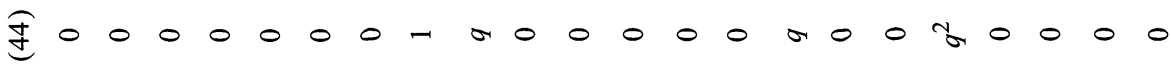

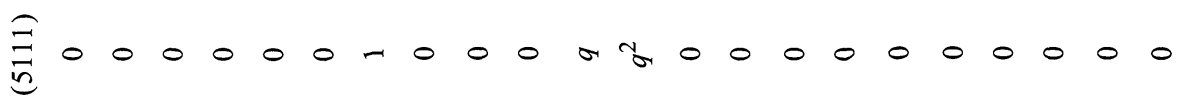

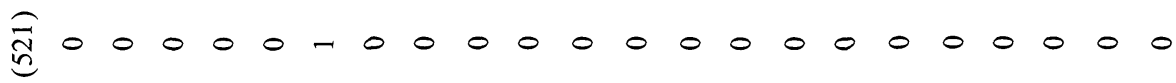

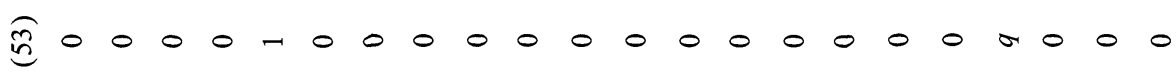

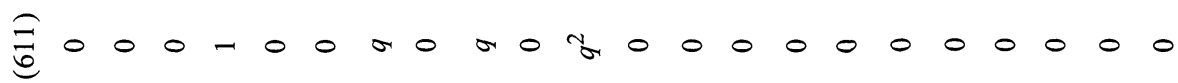
बै $00-0$ a 00000000000000000

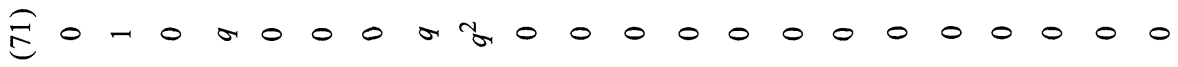

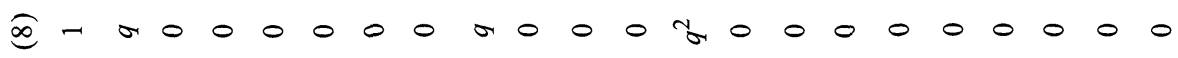

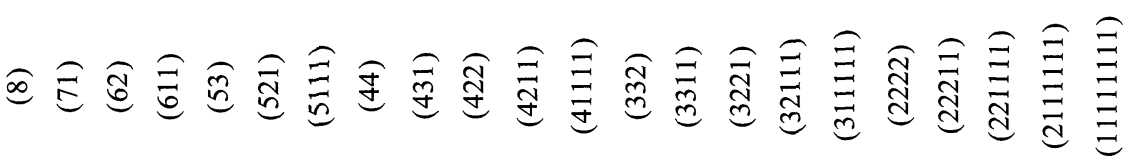


స్ तิ बृ 点 $0000000-00000000000000,00 \% 0000$ $\underset{\sigma}{\Xi} 000000-00000000000000000000000$ ब్రి00000-00 ब్ర0000-0000000000000000000000000 I000-00000000000000000000000000

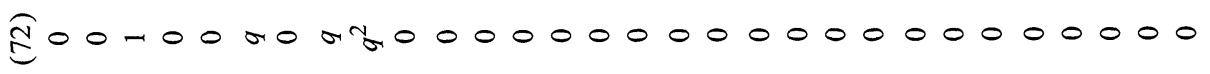
क्ष0-0000000000000000000000000000 O- $-000000,000000000 \% 00000000000$

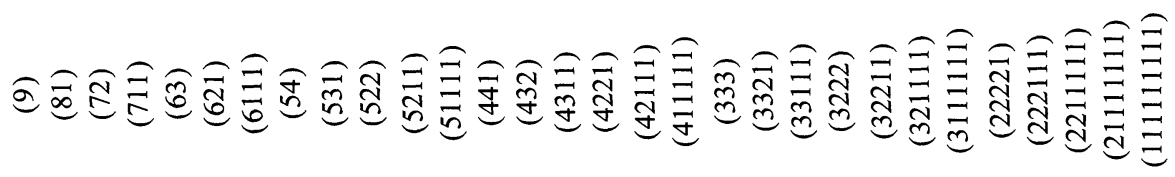


$\underset{\underset{J}{J}}{\stackrel{\Xi}{J}} 00000000000000000000000000-000$

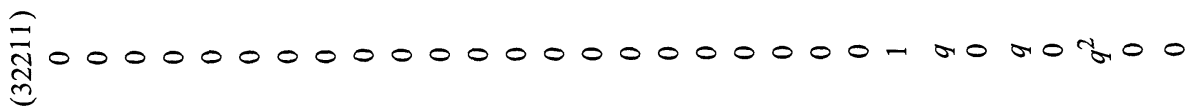

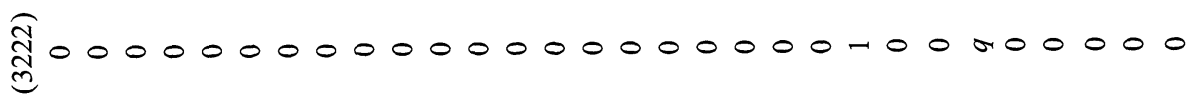
$\underset{\text { छ }}{\Xi} 00000000000000000000-000000000$

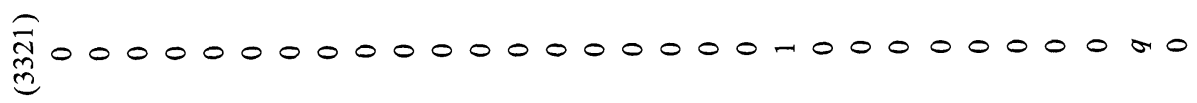

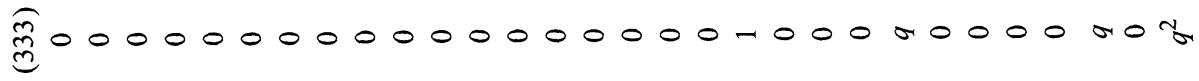

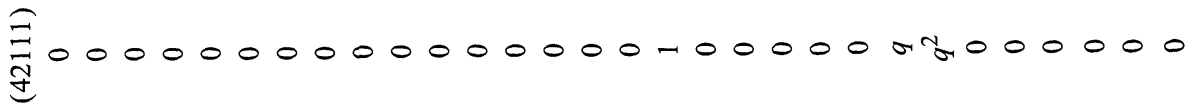
$\underset{\text { ปู }}{\text { I }} 00000000000000-00000000000000$ $\stackrel{\vec{\nexists}}{\stackrel{\Xi}{g}} 00000000000000-000000000000000$ $\underset{\text { d }}{\overparen{J}} 000000000000-0000000000000000$ 焉000000000000-00000000000000000

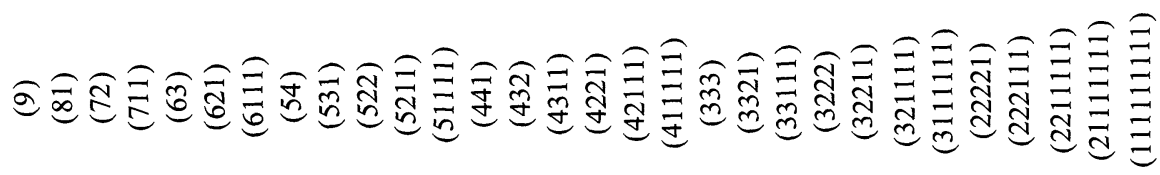




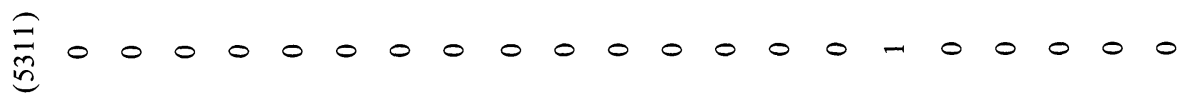

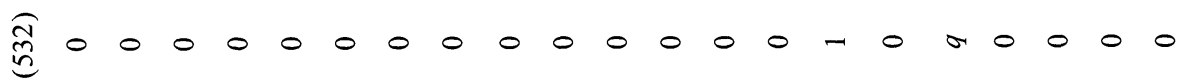

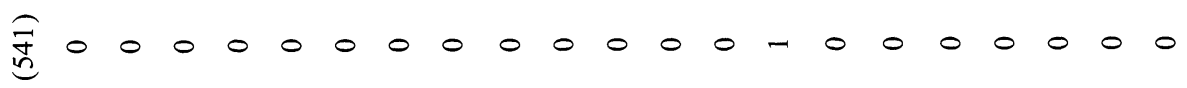

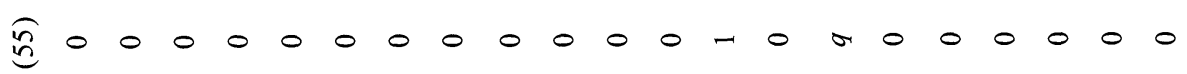

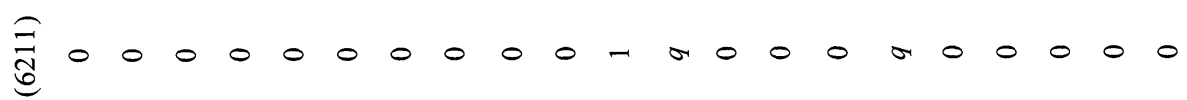

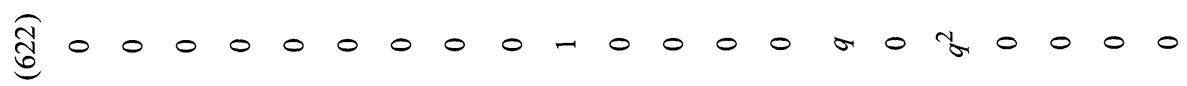

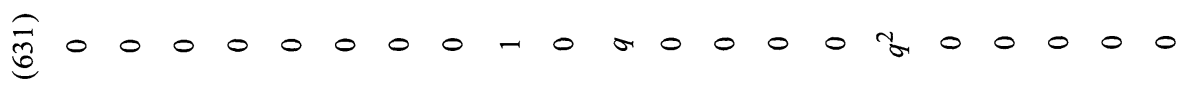

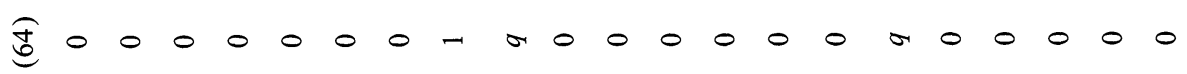

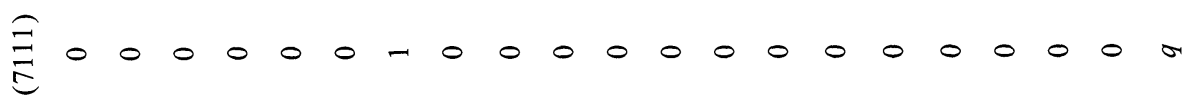

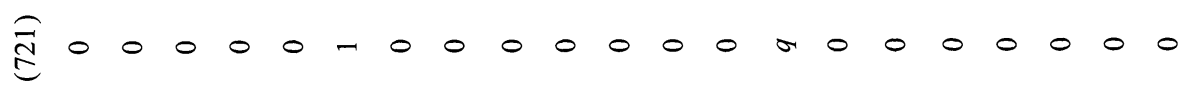

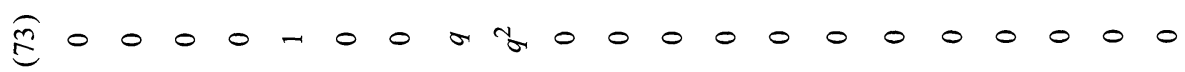

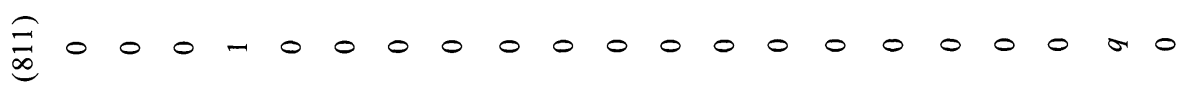

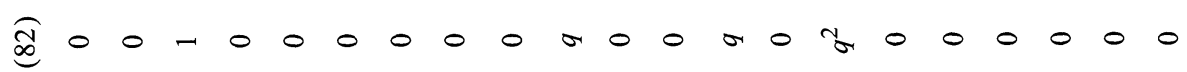

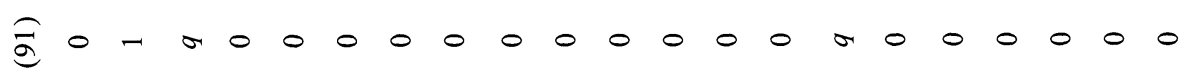

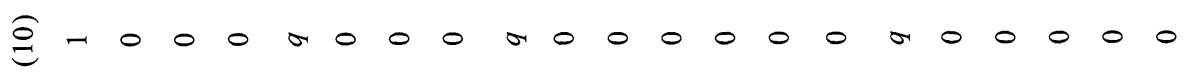

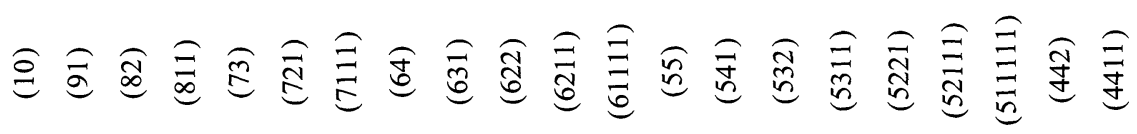




$$
\begin{aligned}
& 00 \text { or } 0000 \text { or } 0 \text { Vro } 0000000000
\end{aligned}
$$

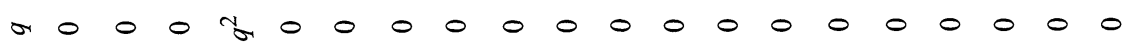

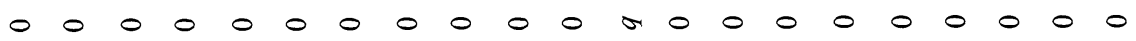

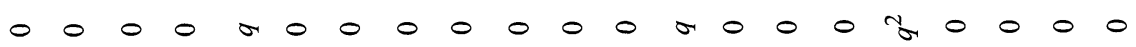

$$
\begin{aligned}
& 0 \text { - V } 00000000000000000000000 \\
& 00000000000000000000000000
\end{aligned}
$$

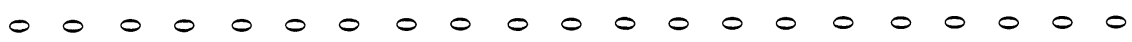

$$
\begin{aligned}
& 000000000 \text { or } 0000000 \text { r. } 0000000
\end{aligned}
$$

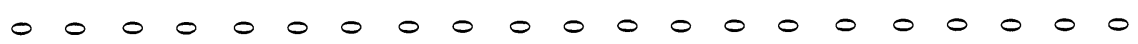

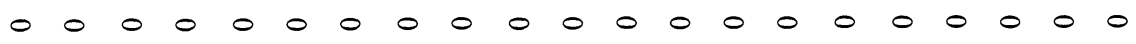

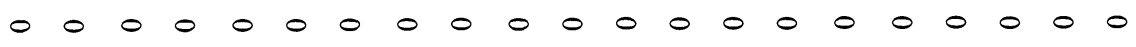

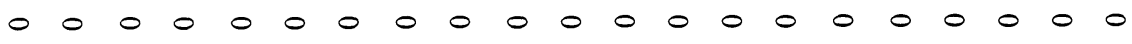

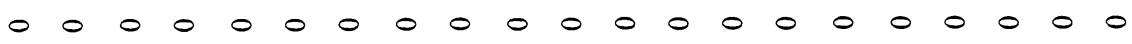

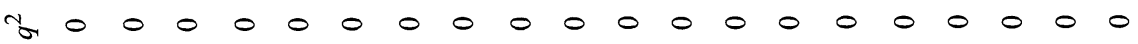

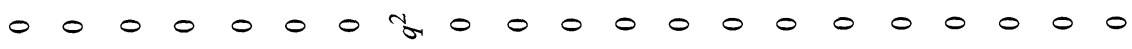

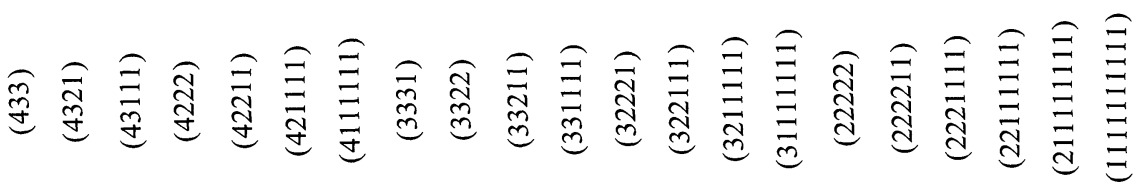




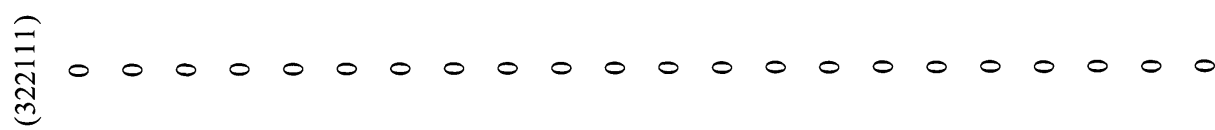

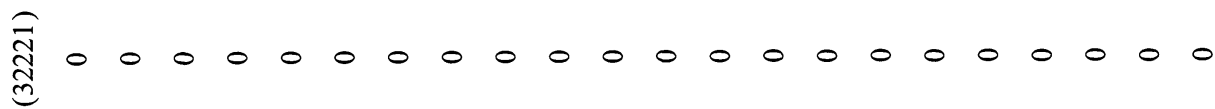

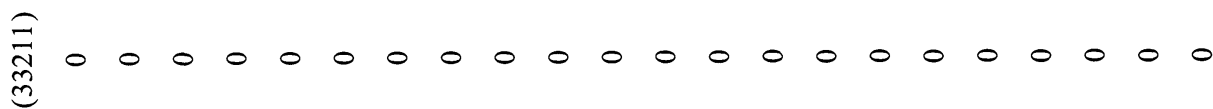

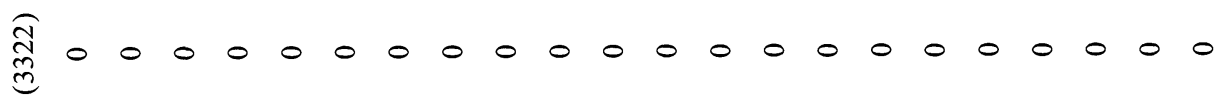

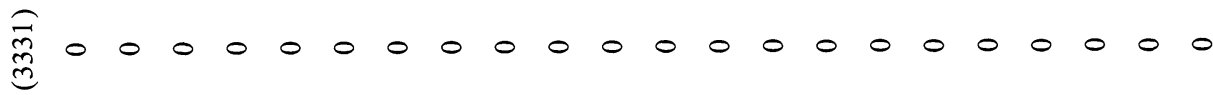

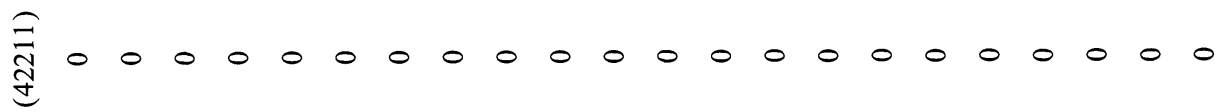

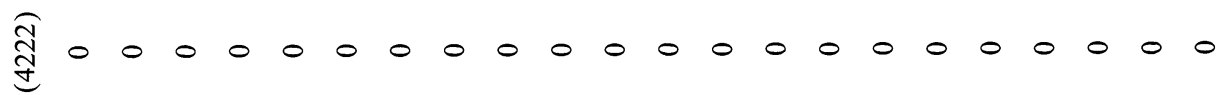

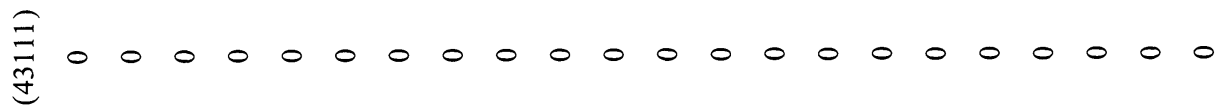

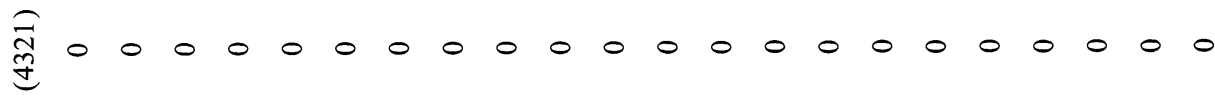

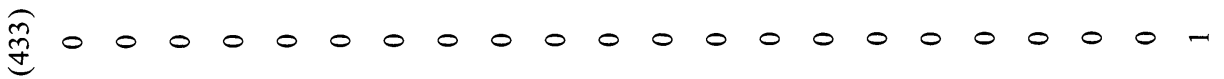

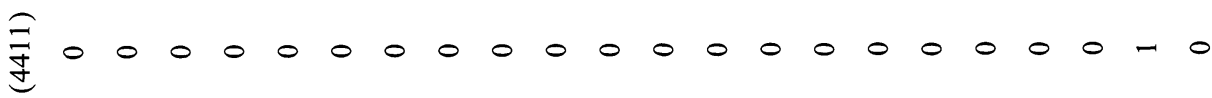

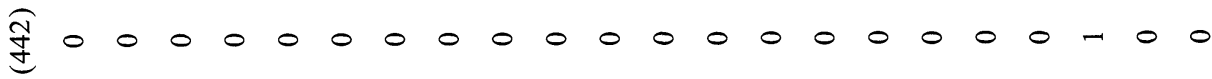

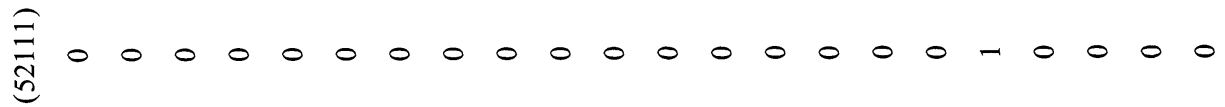

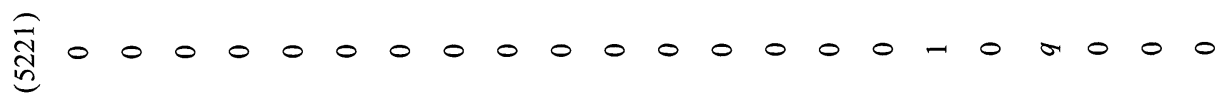

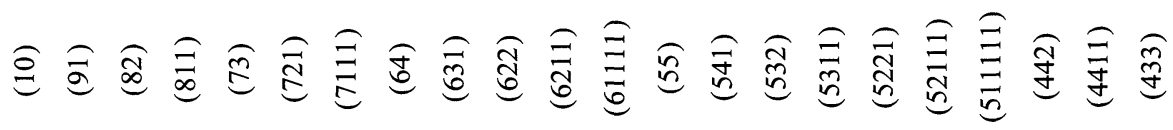




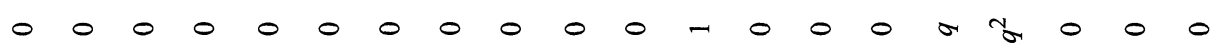

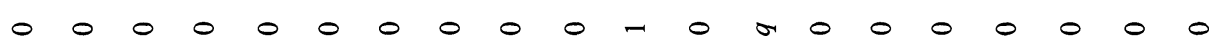

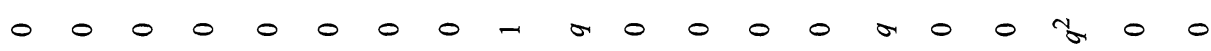

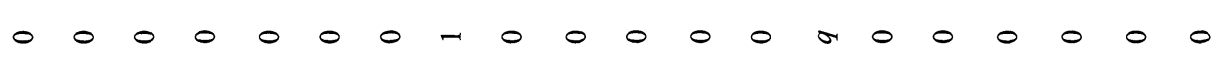

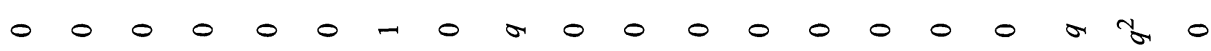

$000-0,0000000 \%$

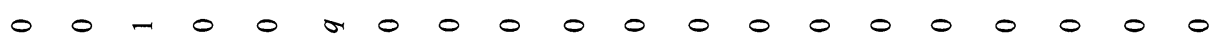

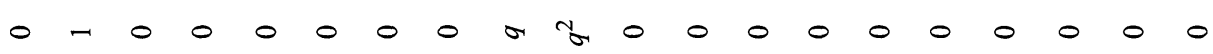

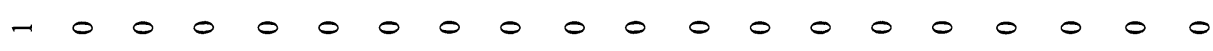

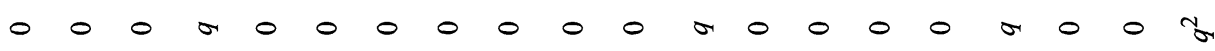

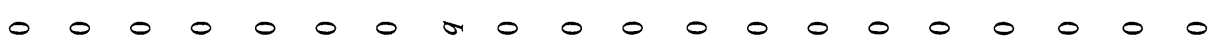

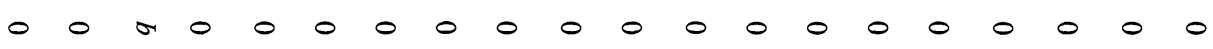

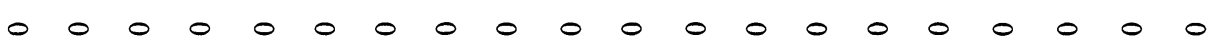

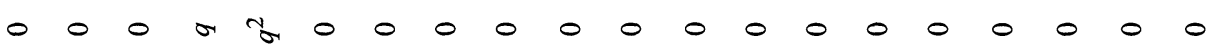

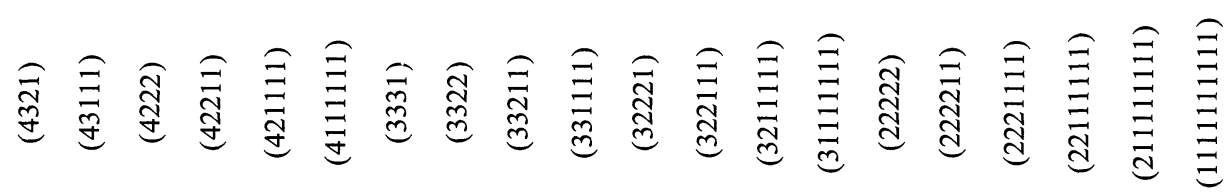


10.6. The Shapovalov form for the basic $U_{q}\left(\widehat{\mathfrak{s}}_{2}\right)$-module
(2) $\stackrel{(2)}{q^{-1}+q}$
$\begin{array}{ccc} & (3) & (21) \\ \text { (3) } & q^{-1}+q & 0 \\ \text { (21) } & 0 & 1\end{array}$
$\begin{array}{ccc}\text { (4) } & q^{-2}+2+q^{2} & q^{-1}+q \\ \text { (31) } & q^{-1}+q & q^{-2}+1+q^{2}\end{array}$
$\begin{array}{cccc} & (5) & (41) & (32) \\ (5) & q^{-2}+1+q^{2} & 0 & 1 \\ (41) & 0 & q^{-1}+q & 0 \\ (32) & 1 & 0 & q^{-2}+1+q^{2}\end{array}$

$\begin{array}{ccccc} & (6) & (51) & (42) & \text { (321) } \\ \text { (6) } & q^{-3}+2 q^{-1}+2 q+q^{3} & q^{-2}+2+q^{2} & q^{-1}+q & 0 \\ \text { (51) } & q^{-2}+2+q^{2} & q^{-3}+2 q^{-1}+2 q+q^{3} & q^{-2}+2+q^{2} & 0 \\ \text { (42) } & q^{-1}+q & q^{-2}+2+q^{2} & q^{-3}+2 q^{-1}+2 q+q^{3} & 0 \\ \text { (321) } & 0 & 0 & 0 & 1\end{array}$
(7) $q^{-3}+q^{-1}+q+q^{3}$
(61)
(52)
(43)
(421)
$\begin{array}{cc}\text { (61) } & q^{-3}+q^{-1}+q+q^{3} \\ \text { (52) } & q^{-1}+q\end{array}$
0
$q^{-2}+1+q^{2}$
$q^{-1}+q$
0
$q^{-3}+2 q^{-1}+2 q+q^{3}$
0
$q^{-1}+q$
0
1
0
$q^{-2}+1+q^{2}$
0
0
(43)
0
1
0
$0 \quad q^{-3}+q^{-1}+q+q^{3}$

10.7. The Shapovalov form for the basic $U_{q}\left(\widehat{\mathfrak{s l}}_{3}\right)$-module
(2) (11)
(2) 10
(11) $0 \quad 1$
(3) (21)
(3) $q+q^{-1}$
(4) (31) (22)
(211)
(11) 0 - 1
(21) $1 \quad q+q^{-1}$
$\begin{array}{lcc}\text { (4) } & q+q^{-1} & 0 \\ (31) & 0 & 1 \\ (22) & 1 & 0 \\ (211) & 0 & 0\end{array}$
$\begin{array}{cc}1 & 0 \\ 0 & 0 \\ q+q^{-1} & 0 \\ 0 & 1\end{array}$

$\begin{array}{cccccc} & (5) & (41) & (32) & (311) & (221) \\ (5) & q+q^{-1} & 0 & 0 & 0 & 1 \\ (41) & 0 & q+q^{-1} & 1 & 0 & 0 \\ (32) & 0 & 1 & q+q^{-1} & 0 & 0 \\ (311) & 0 & 0 & 0 & 1 & 0 \\ (221) & 1 & 0 & 0 & 0 & q+q^{-1}\end{array}$




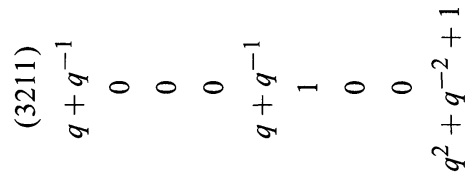

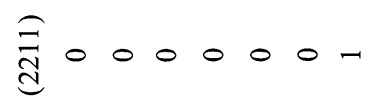

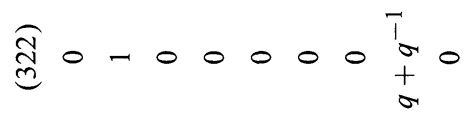

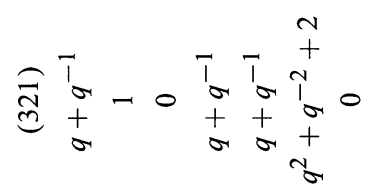

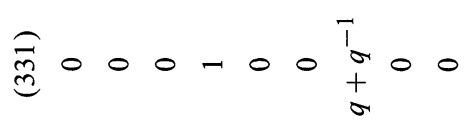

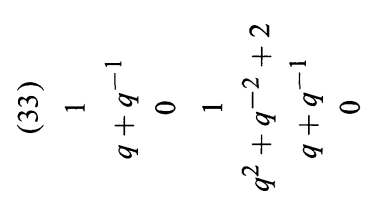

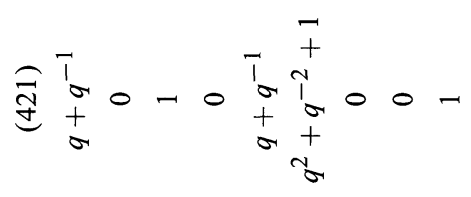

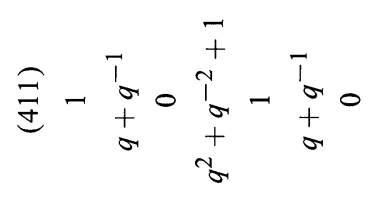

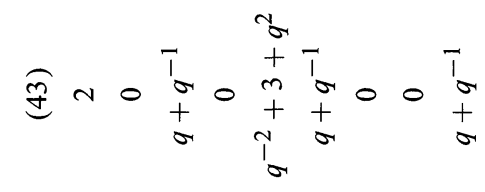

$$
\begin{aligned}
& \text { Iิ } 00-0000
\end{aligned}
$$

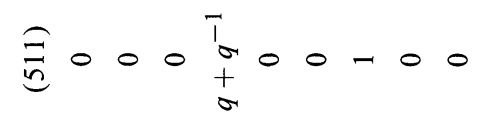

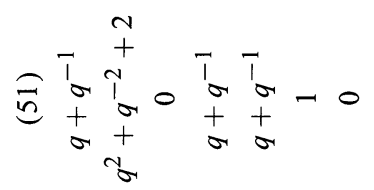

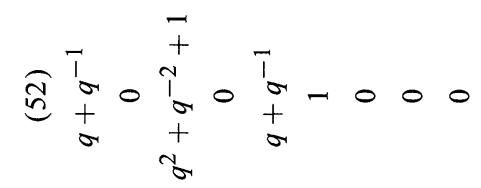

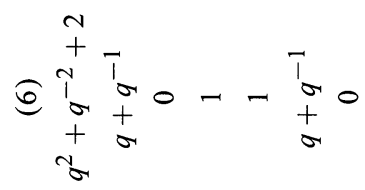

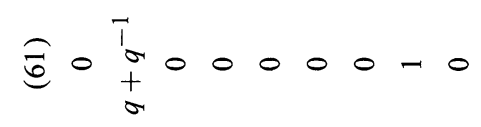

$$
\begin{aligned}
& \text { }
\end{aligned}
$$

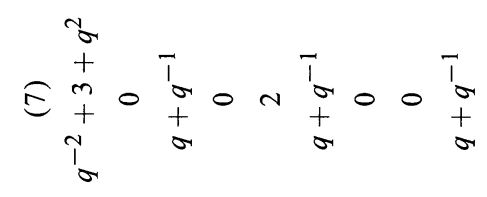

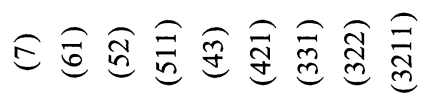


Acknowledgements We would like to thank G. James and A Mathas for fruitful discussions and for communicating to us some unpublished decomposition matrices. Thanks are also due to $\mathrm{C}$ Bessenrodt who brought Kleshchev's results to our knowledge and who raised the question of defining a $n$-reduced quotient, and to $\mathrm{R}$ Rouquier who suggested the interpretation of Sect. 9. Also we are grateful to I Cherednik, O. Foda, D Kazhdan, A Kleshchev and A Zelevinsky for stimulating discussions on the matters of this paper. Finally, we thank the referee for pointing out that the proofs of some statements of Sect. 6 were incomplete in the first version of this paper.

\section{References}

1. Andrews, GE., Olsson, J.B.: Partition identities with an application to group representation theory J. Reine Angew. Math. 413, 198-212 (1991)

2 Ariki, S.: On the decomposition numbers of the Hecke algebra of $G(m, 1, n)$ Preprint 1996

3. Bessenrodt, C, Olsson, J.B : On Mullineux symbols. J Comb. Theory Ser. A 68, 340-360 (1994)

4. Curtis, C., Reiner, I.: Methods of representation theory with applications to finite groups and orders. Vol. 1, New York: Wiley, 1981

5 Date, E., Jimbo, M, Kuniba, A., Miwa, T., Okado, M.: Paths, Maya diagrams, and representations of $\widehat{\mathfrak{s l}}(r, C)$ Adv. Stud. Pure Math. 19, 149-191 (1989)

6. Davies, B., Foda, O, Jimbo, M., Miwa, T, Nakayashiki, A : Diagonalization of the XXZ Hamiltonian by vertex operators. Commun. Math. Phys 151, 89-153 (1993)

7. Dipper, R., James, G.: Representations of Hecke algebras of general linear groups Proc. Lond. Math. Soc. 52, 20-52 (1986)

8 Dipper, R., James, G.: Blocks and idempotents of Hecke algebras of general linear groups. Proc. Lond. Math Soc. 54, 57-82 (1987)

$9 \mathrm{Du}, \mathrm{J}$ : Canonical bases for irreducible representations of quantum $G L_{n}$ Bull. Lond. Math. Soc. 24, 325-334 (1992)

10. Du, J.: Canonical bases for irreducible representations of quantum $G L_{n}$ II. J Lond. Math Soc. 51, 461-470 (1995)

11 Duchamp, G, Krob, D, Lascoux, A., Leclerc, B., Scharff, T, Thibon, J -Y.: Euler-Poincaré characteristic and polynomial representations of Iwahori-Hecke algebras. Publ. RIMS, Kyoto Univ 31, 179-201 (1995)

12 Ford, B., Kleshchev, A.S.: A proof of the Mullineux conjecture Preprint 1994

13. Hayashi, $\mathrm{T}: q$-analogues of Clifford and Weyl algebras - Spinor and oscillator representations of quantum enveloping algebras. Commun Math. Phys. 127, 129-144 (1990)

14. James, G.: The decomposition matrices of $G L_{n}(q)$ for $n \leqq 10$ Proc Lond Math Soc 60, 225-265 (1990)

15 James, G, Kerber, A.: The representation theory of the symmetric group. Reading, MA: Addison-Wesley, 1981

16. James, G., Mathas, A : Hecke algebras of type $A$ with $q=-1$ Preprint 1995

17. James, G., Mathas, A.: A $q$-analogue of the Jantzen-Schaper theorem Preprint 1995

18. Kac, V.: Infinite dimensional Lie algebras Cambridge: Cambridge University Press, 1990

19 Kashiwara, M.: On crystal bases of the $q$-analogue of universal enveloping algebras Duke Math J 63, 465-516 (1991)

20. Kashiwara, M.: Global crystal bases of quantum groups. Duke Math. J. 69, 455-485 (1993)

21 Kashiwara, M.: Crystal bases of modified quantized enveloping algebra. Duke Math. J. 73, $383-413$ (1994)

22. Kazhdan, D., Lusztig, G.: Affine Lie algebras and quantum groups. Int Math. Res. Not. 2 , 21-29 (1991)

23. Kleshchev, A S.: Branching rules for the modular representations of symmetric groups I. J. Algebra (to appear)

24. Kleshchev, A S.: Branching rules for the modular representations of symmetric groups II. J. Reine. Angew. Math. 459, 163-212 (1995)

25 Kleshchev, A S.: Branching rules for the modular representations of symmetric groups III; Some corollaries and a problem of Mullineux. J Lond Math. Soc. 2 (1995)

26. Lakshmibai, V.: Bases for quantum Demazure modules II. Proc. Symp. Pure Math 56, part 2, 149-168 (1994) 
27. Lascoux, A : Leclerc, B, Thibon, J -Y.: Ribbon tableaux, Hall-Littlewood functions and unipotent varieties In: "Algebraic Combinatorics, Rencontre 12-16 March 1995, Saint-Nabor, Ottrott, France," Université Louis Pasteur, IRMA, Strasbourg 1995, pp 183-207

28. Lascoux, A.: Leclerc, B., Thibon, J.-Y.: Une conjecture pour le calcul des matrices de décomposition des algèbres de Hecke de type $A$ aux racines de l'unité C R. Acad. Sci Paris 321, 511-516 (1995)

29. Lascoux, A.: Leclerc, B , Thibon, J-Y : Crystal graphs and $q$-analogues of weight multiplicities for the root system $A_{n}$. Lett. Math Phys 35, 359-374 (1995)

30. Leclerc, B, Thibon, J.-Y.: Canonical bases of $q$-deformed Fock spaces Internat. Math Research Notices 9 (1996)

31 Lusztig, G : Modular representations and quantum groups. Contemp. Math. 82, 59-77 (1989)

32. Macdonald, I G.: Symmetric functions and Hall polynomials 2nd edition, Oxford: Oxford Univ. Press, 1995

33 Martin, S.: Schur algebras and representation theory Cambridge: Cambridge University Press, 1993

34 Martin, S : On the ordinary quiver of the principal block of certain symmetric groups. Quart J Math. Oxford Ser. 40, 209-223 (1989)

35. Martin, S : Ordinary quivers for symmetric groups Quart. J. Math Oxford Ser. 41, 72-92 (1990)

36 Misra, K.C, Miwa, T.: Crystal base of the basic representation of $U_{q}\left(\widehat{\mathfrak{s l}}_{n}\right)$. Commun. Math. Phys 134, 79-88 (1990)

37. Mullineux, $\mathrm{G}$ : Bijections of $p$-regular partitions and $p$-modular irreducibles of the symmetric groups J. Lond Math. 20, 60-66 (1979)

38. Richards, M.J : Some decomposition numbers for Hecke algebras of general linear groups.

39. Robinson, G De B.: Representation theory of the symmetric group. Edimburgh, 1961

40 Zelevinsky, A : Representations of finite classical groups, a Hopf algebra approach. Lect. Notes in Math 869, Berlin-Heidelberg-New York: Springer, 1981

\section{Supplementary reference}

[Gr] Grojnowski, I.: Representations of affine Hecke algebras (and affine quantum $G L_{n}$ ) at roots of unity Internat. Math Research Notices 5, 215-217 (1994) 
